

\section{DISCLAIMER}

This report was prepared as an account of work sponsored by an agency of the United States Government. Neither the United States Government nor any agency Thereof, nor any of their employees, makes any warranty, express or implied, or assumes any legal liability or responsibility for the accuracy, completeness, or usefulness of any information, apparatus, product, or process disclosed, or represents that its use would not infringe privately owned rights. Reference herein to any specific commercial product, process, or service by trade name, trademark, manufacturer, or otherwise does not necessarily constitute or imply its endorsement, recommendation, or favoring by the United States Government or any agency thereof. The views and opinions of authors expressed herein do not necessarily state or reflect those of the United States Government or any agency thereof. 


\section{DISCLAIMER}

Portions of this document may be illegible in electronic image products. Images are produced from the best available original document. 


\section{LEQAL NOTICE}

This report was prepared as an account of work aponsored by the United States Government. Neither the United States nor the United States Department of Energy, nor any of their employees, nor any of their contractors, subcontractors, or their employees, makes any warranty, express or implied, or essumes any kgal liability or responsibility for the accuracy. completeness or usefulness of any information, apparatus, product or process disclosed, or represents that its use would not infringe privately owned rights.

Printed in the United States of America Available from

National Technical Information Service

U.S. Department of Commerce

5285 Port Royal Road

Springfield, VA 22161

Price Code: 108 


\title{
ANNOTATED RESEARCH BIBLIOGRAPHY FOR \\ GEOTHERMAL RESERVOIR ENGINEERING
}

by

G. A. Sudol, R. F. Harrison, and H. J. Ramey, Jr.

\author{
Prepared for: \\ Lawrence Berke ley Laboratory \\ University of California \\ Berkeley, California 94720
}

Submitted by:

Terra Tek. Inc.

University Research Park

420 Wakara Way

Salt Lake City, Utah 84108

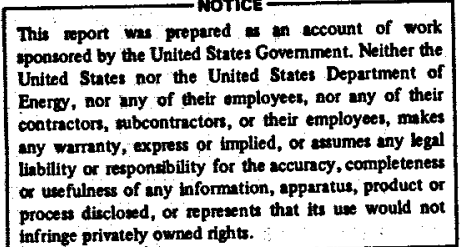

process disclosed, of represents
infringe privetely owned rights. 


\section{ACKNOWLEDGMENTS}

This work was performed under purchase order no. 3473402 for Lawrence Berkeley Laboratory, Berkeley, California, and the U.S. Department of Energy under contract W-7405-ENG-48.

The guidance and encouragement of John H. Howard, Werner J. Schwarz and Beverly Strisower of the Earth Sciences Division, Lawrence Berkeley Laboratory are gratefully acknowledged. 
TABLE OF CONTENTS

Page

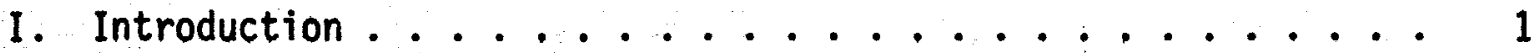

1.1 Justification for the Bibliography .......... 1

1.2 Definition of Geothermal Reservoir Engineering and

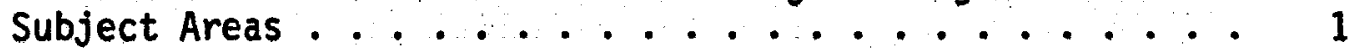

1.3 Historical Development of Geothermal Reservoir

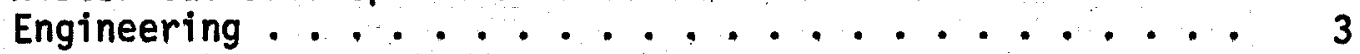

1.4 Data Acquisition Techniques ........... 6

II. Subject Areas ...................... 7

2.1 Formation Evaluation ............. . . 7

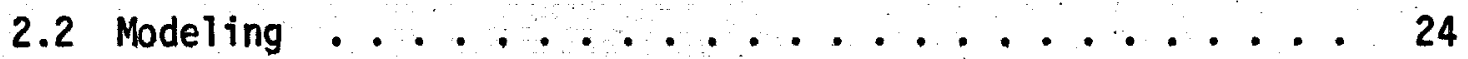

2.3 Exploitation Strategies ............. 78

2.4 Interpretation of Production Trends . . . . . . . . 91

III. Reference List .............................. 99

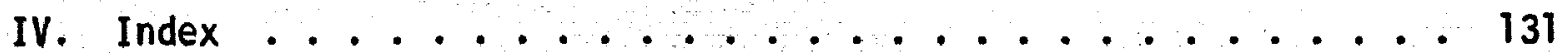




\section{INTRODUCTION}

1.1. Justification for the Bibliography

Researchers review technical literature in order to avoid duplication of research. It has been estimated that approximately one-tenth of the research and development funds expended in the United States have been applied to areas resulting in a duplication of effort. Kertes ${ }^{*}$ has attributed this to inadequate literature review.

Despite efforts of conscientious researchers, the current volume of published material makes it virtually impossible to review all relevant publications in a given field. To keep abreast of research within a particular area and to obtain a specific answer to a problem, new retrieval methods have been developed. These techniques include abstracting and indexing of journal articles, developing computer-based storage and retrieval systems, developing methods for reviewing articles, and establishing technical information data centers.

An up-to-date annotated bibliography has been prepared which will assist in avoiding duplication of geothermal reservoir engineering research and serve as a definitive record of the progress and current status of the subject. The bibliography includes English, Italian, Russian, and Japanese language pubitications.

\subsection{Definition of Geothermal Reservoir Engineering and Subject Areas}

The accelerated research and development effort in alternative energy forms in recent years has resulted in considerable progress in many aspects of geothermal exploration and development, including the development of engineering methodology focusing on the behavior of reservoirs under the perturbed conditions of production. Much of the work is based on the well-established nomenclature and methodologies of the oil and gas industry, hydrology, and hydrogeology. Additional basic input from physical, chemical, and nuclear sciences is being adapted to geothermal conditions to establish specific techniques, necessitated by the unique and varied features of geothermal systems. Natural geothermal resources are found in a wide variety of geological and hydrological environments, each system exhibiting its own thermal and chemical characteristics. Inevitably utilization must be designed to fit the specific characteristics of a given resource, and the reservoir engineer will have to fully understand its nature before he can select with any confidence a cost-effective strategy for its exploitation.

To develop a useful background for generating a conceptual model

${ }^{\star}$ Kertesz, F., The Information Center Concept, Oak Ridge, Oak Ridge National Laboratory ORNL-TM-2281 (1968), p. 12. 
of a particular system under study, the engineer must understand the general physical processes associated with geothermal systems. Then he must assess the physical properties of the rock and fluid and determine the structural features of the reservoir. A mathematical or physical model is then developed using the defined physical properties along with the identified initial and boundary conditions. This model is used to assess initial production strategies. The actual reservoir response under production is then used to update and refine the model, allowing extrapolations of performance to be made with greater confidence.

This general chronological sequence of events has been used to outline the following four broad categories under which the subject matter for the bibliography was collected.

\section{- Formation Evaluation}

The performance of a mathematical or physical reservoir model will match the real case only if accurate estimates of the in situ formation parameters such as porosity, permeability, thermal conductivity, etc., are available. Therefore the well testing, well logging, and laboratory rock property evaluation techniques commonly used are an integral part of the reservoir engineering program.

To maintain the emphasis of the bibliography on reservoir performance interpretation, literature discussing measurement techniques is not included. For example, a citation with emphasis on the operation or calibration of a downhole logging tool is not included; however, one discussing the estimates of a physical reservoir characteristic obtained by using this technique is included. In essence, the rationale for subject retrieval is based on documentation of field test results rather than the data gathering techniques used to generate these results.

\section{- Reservoir Modeling}

Analytical, numerical, and physical (scale) models have been developed to simulate the mass, heat, and species transport characteristics of geothermal systems in the natural state and to evaluate the behavior of these systems under various production strategies. The development of a realistic reservoir model is a major goal of the geothermal reservoir engineering program.

\section{- Exploitation Strategies}

Literature retrieval within this classification is focused on reservoir management and production enhancement. This includes topics such as stimulation, production-injection strategies, and well spacing. Those topics related to production engineering, such as piping network design and power station siting and design, are not included in the bibliography. 


\section{- Interpretation of Production Trends}

Valuable information can be obtained on the nature of a geothermal reservoir by monitoring changes in reservoir characteristics during production. For example, periodic gravity surveys can give an indication of the fluid depletion and recharge rates. Localized resistivity changes can indicate areas of boiling due to pressure drawdown. Injection of tracers and subsequent monitoring of produced fluids can indicate patterns of fluid movement; and changes in fluid chemistry can indicate changes in reservoir enthalpy and the origin of produced fluids. These, together with analyses of trends in pressure drawdown and heat and mass fluxes, reveal the long-term reservoir deliverability characteristics. Reservoir models can be refined by adjusting the model to fit past production trends and, as a result, extrapolation into the future can be made with more confidence.

Only literature which emphasizes the interpretation of trends in reservoir characteristics during production is included in the bibliography. Discussions of the geologica 1, geophysical, and geochemical techniques used in exploration are not included.

Some subjectiveness has been necessary in evaluating the applicability of material for the bibliography. The reservoir is the focus of any geothermal development and Geothermal Reservoir Engineering must not be defined so broadly that it includes all technical aspects of geothermal development. In determining the bibliography scope as outlined above, reservoir engineering was identified as the general topic connecting exploration science and production engineering. Exploration science deals with surface geophysical, geological, and geochemical techniques used to find and assess regional aspects of the reservoir, and production engineering deals with the hardware of fluid collection, transmission, and processing. This general classification was kept in mind when screening individual citations for the bibliography.

\subsection{Historical Development of Geothermal Reservoir Engineering}

Although major development of geothermal resources for power generation began at Lardere110, Italy at the turn of the century, it was not until the late $1940^{\prime} \mathrm{s}$ and early $1950^{\prime}$ 's that the scientific community became interested in the quantitative aspects of heat and mass transfer processes in these natural systems. This was in part due to renewed interest in geothermal development in post-war Italy and to new projects at The Geysers, California, and Wairakei, New Zealand.

A hydrothermal system obviously has some basic similarities with $0 i 1$ and gas reservairs and groundwater aquifers. The principal unifying factor is that all-deal with the transport of fluids through parent rock matrices. As a result, much of the applied methodology and terminology used in geothermal reservoir engineering has origins within the fields of hydrology and $0 i l$ and gas reservoir engineering. For example, most 
geothermal systems (a11 hydrothermal systems) are connected in some way to the regional groundwater. Consequently, forced convection in moderate to low temperature systems is often treated on the basis of simple hydrologic models.

Quantitative applied reservoir engineering methods were developed for production management of oil and gas reservoirs in the late 1940's and early 1950's. Methodology has been developed for interpreting individual oil well performance in terms of physical and structural properties of the reservoir, for matching the performance history of a reservoir and for developing simulators from which predictions of the future performance can be made and the relative merits of different exploitation strategies assessed. These methods continue to be refined to develop sophisticated production strategies ensuring maximum recovery in specific cases. The application of this methodology to hydrothermal systems has helped to explain the practical aspects of reservoir performance.

Although not directed specifically at geothermal systems, the studies by Lapwood [1948] and Horton and Rogers [1945] on the onset of thermal convection in a porous layer heated from below were the first quantitative analyses of the physical processes important to natural hydrothermal systems. These studies were based on the classical work of Rayleigh whose analys is of natural convection in a viscous liquid resulted in the definition of the convection-determining dimensionless group now appropriately called the Rayleigh number. Wooding [1957] reported the first application of theory to a real hydrothermal process. He compared mathematically-generated temperature profiles with measured data taken from the Wairakei geothermal field in New Zealand.

The initial analytical work was supplemented by combined experimental and mathematical investigations of natural thermal convection. Hele-Shaw cells were particularly useful for studying steady-state free and combined free-forced convection motions. For example, Elder [1960] and Wooding [1957] visualized natural convection under a variety of conditions and used the results to verify accompanying mathematical approaches.

The early work defined the basic physical processes governing natural thermal convection in a porous media and quantified the magnitude of convection and heat transfer that would be generated under a given set of determining physical conditions. However, idealized cases with simplified physical properties and mathematically tractable boundary conditions were generally considered. The results of such analyses for steady state conditions could not be readily applied to analyses of the long-term consequences of heat and mass extraction from a thermodynamic system with nonisotropic structural properties and finite reservoir size.

For the purposes of geothermal development which seeks to optimize energy extraction over a 25 to 30-year useful plant life, the natural geologic history of the system is not of immediate importance and, in many cases, the reservoir can be considered as a contained body of hot fluid which is produced by forced convection. 
Whiting [1969] applied lumped-parameter material and energy balances commoniy used in oil and gas reservoir analysis to demonstrate probable production histories for various hydrothermal systems. James [1965] also used the lumped-parameter model approach to show that drawing steam from a shallow boiling zone was a more effective production strategy than drawing from a compressed water zone of a Wairakei-type hot water system.

These zero-order approaches could not account for spatial variations in reservoir conditions as a result of withdrawal from localized sinks (wel1s) in the reservoir. One-, two-, and three-dimensional distributed-parameter modeling of geothermal reservoirs began in the early 1970's. These models are aimed at simulating reservoir performance in forced convection (production) situations. Line and point sinks (production wells) and sources (injection wells) and boiling with resulting nonisothermal flow are considered and reservoir histories determined as a function of withdrawal rates. Because of the resulting highly nonlinear equations, numerical solution techniques have been extensively used.

The use of these techniques in site-specfic studies has increased steadily and models describing the behavior of Wairakei and Broadlands, New Zealand; Larderello, Italy; East Mesa, California; Cerro Prieto, Mexico; Matsukawa, Japan; and other natural geothermal systems have been developed. For long-term usefulness, all of the models deal with phenomena peculiar to combined natural and forced convective heat transfer. Topics of particular relevance to geothermal situations are nonisothermal boiling, steam/water capillarity, fluid/rock heat transfer, and chemical species transport. Basic experimental and theoretical research programs are under way to investigate these fundamental properties and evaluate their relevance to specific cases [Hundsbedt (1977), Atkinson (1976), Arihara (1974), Cady (1972)].

In addition to field-scale simulations, individual well performance evaluation is important to practical day-to-day field operations. Well evaluation techniques developed early in the geothermal industry relied mainly on wellhead measurements with qualitative input from downhole measurements of temperature and pressure under static and reversed-pumping situations. The diagnostic features of pressure-transient testing were recently introduced from the $0 i 1$ and gas industry and some applications have been reported. Extensive use of these techniques is limited by the difficulty in acquiring data due to the severe downhole environment to which the instrumentation is subjected in geothermal wells. In addition, there is a possibility that these procedures may not be applicable where nonisothermal flow is possible. Formation thickness and boundaries are not well defined, and in many cases fluid flow through fractures is the dominant transport process, so well testing techniques are now being developed which include the effects of boiling, nonisothermal fluid flow, and steam/water relative permeability.

Present geothermal exploitation is 1 imited to hydrothermal systems where natural circulation of water provides a means for extracting 
reservoir heat. In some cases, however, the porosity and permeability is very low and natural convection cannot be relied upon to transport heat. Future geothermal developments will be aimed at extracting the heat from such systems. Enhanced recovery methods widely used in the oil industry, such as hydrofracturing, acidizing, explosive stimulation, etc., are being considered for these applications. An extensive field program is currently under way to determine the feasibility of creating downhole heat exchange surfaces in hot dry rock.

The final key to continued development and refinement of geothermal reservoir engineering techniques relates directly to the experience that can be gained from current and future research. Most researchers feel that it is necessary to monitor the response of a perturbed field before a realistic simulator can be evolved. The Larderello, Geysers, and Wairakei developments have provided a data base from which general management procedures, interpretive techniques, and conceptual models for producing geothermal systems have been derived [James (1968); White (1957)]. As more geothermal areas are put into production, their general similarities as well as their unique features will become more apparent.

\subsection{Data Acquisition Techniques}

Literature used in the bibliography compilation was retrieved by means of computerized data bases, published bibliographies, and abstract services. The material was collected from various sources including major colleges and universities, U.S. and foreign government agencies, industry, conferences, and special interest groups.

Initially, 2,000 items were retrieved, evaluated, and selected for subject relevance. Fewer than twenty-five percent of the retrieved items were finally included in the annotated bibliography.

The scope of the literature covered included materials from 1949 to 1978, though most sources were published from 1960 to 1978 due to increased interest and research efforts in the geothermal field. The coverage was international and foreign-language literature was retrieved though all annotated articles are in English. 


\section{SUBJECT AREAS}

\subsection{Formation Evaluation}

Estimates of the physical characteristics of the reservoir rock and fluid are necessary initially to define the structural controls of the system, to identify the important thermomechanical processes, and subsequentiy, as input for mathematical models of anticipated reservoir performance. An analysis of the global associations of geothermal areas is given by Facca [1964]. He outlines the gross tectonic and volcanic structural associations of geothermal systems and postulates on a number of possible mechanisms of heat transfer.

The preliminary conceptual model of a reservoir is normally based on the results of surface exploration surveys. A well is the only means, however, of obtaining direct information on the reservoir, and the siting of test holes for maximum effect is of paramount importance. Banwell [1964] stresses the importance of synthesizing all available surface geological, geophysical, and geochemical information before choosing a test hole location. The largely qualitative concepts are then correlated with drilling results where recorded lithology and geophysical logs can further delineate the stratigraphy of the system. Kunze [1975], Schroeder [1976], and Black [1975] use well data coupled with geological and geophysical exploration results to identify potential production zones in the Raft River, Salton Sea, and East Mesa geothermal areas. Wainwright [1970] outlines a reverse-flow pump test which is used to identify production zones and their approximate permeability.

Well flow and transient response tests are perhaps the most useful reservoir diagnostic tools. Chen [1974] reviews the methods commonly used by the geothermal industry and discusses background theory from the oil and gas industry from which these methods have evolved.

Flow rate tests, where bore output versus well head pressure response are established, are the simplest and most important well tests for candidate production wells. Well flow rate measurement procedures and equipment requirements are discussed by Wainwright [1970]. These borehole flow characteristics can often be used qualitatively as an interpretive tool. For example, James [1970] applies a simple test to borehole characteristic curves to determine whether the formation supplying the well is fracture or matrix permeability controlled and outlines a test method for estimating formation enthalpy. Chen [1978] describes the nature of the Hawaii geothermal reservoir on the basis of the general characteristics of the well flow data.

An important formation diagnostic tool is the pressure transient test. Ramey [1975] reviews pressure-transient well test techniques cormonly used in the oil and gas industry and submits that direct application to geothermal conditions is often possible. However, he warns against reckless use in cases where two-phase nonisothermal flow is 
possible. Gulati [1975] supports this view and outlines some areas where the standard analysis can lead to errors under geothermal conditions. Rice [1976], Garg [1978], and Horne [1978] have investigated the relationship between two-phase formation flow and well response and have used mass momentum and energy conservation equations to define steamwater relative permeabilities for boiling flow in porous media. Moench [1978] considers the effects of vapor pressure lowering on well test results in vapor-dominated systems. The results show that drawdown curves are virtually unchanged from standard curves whereas the buildup curve differs significantly. Techniques which include the effects of boiling two-phase flow, fracture conductivity, and heat transfer will be essential to the general usefulness of well testing techniques in geothermal conditions.

Standard well-test techniques have been used in cases where the assumption of isothermal flow through a porous matrix is valid. Morse [1978], Schroeder [1976], and Witherspoon [1976] have provided realistic values of local and general reservoir properties such as diffusivity, transmissivity, and capacity for the Salton Sea, East Mesa, and Raft River reservoirs, using standard techniques. Bernard [1975] has applied the techniques to geothermal geopressured aquifers and stresses the effects of significant reservoir drive mechanisms such as rock compressibility and water influx from lower permeability shales.

In some cases, where the accuracy of absolute values is suspect, the pressure-transient techniques have been useful in delineating variations within the reservoir [Celati, 1957]. Even where fracture permeability is very evident, the porous media assumption can produce useful results. McNabb [1975] agrees that permeability in the Wairakei geothermal field is fracture-dominated; however, he submits that Darcy-type permeability can be defined on a macroscopic scale. He proposed a number of methods for estimating permeability based on observed effects of heat and mass transfer locally within wells and on the larger reservoir scale.

Many important reservoir parameters have been successfully measured in the laboratory. Permeabilities and porosities have been estimated from laboratory core measurements, but the investigators are careful to correlate the experimental results with other reservoir data. Ramey [1974] defines the various constituent properties of rock and fluid which are necessary inputs for mathematical representations of reservoirs and outlines a number of laboratory techniques for estimating parameters such as thermal conductivity, diffusivity, porosity, wetability, relative permeability, and the thermodynamic properties of formation fluids.

Other investigators have studied the combined effects of temperature and pressure on rock properties [Somerton, 1976; Sanya 1, 1974; Roegiers, 1974]. It appears from these studies that reservoir properties could change significantly as formation temperatures and pressures are altered by production. Bodvarsson [1975] uses an elastic model for rock to show that changes in ground surface can be used to monitor subsurface temperature changes. 
Many reservoir parameters necessary for quantitative modeling of oil and gas systems are heuristically determined, at least initially, on the basis of past experience and data generated in similar reservoir situations. The same approach will eventually be possible in geothermal situations as more fields are developed and the subsequent data bank is increased.

Atkinson, P. and others. "We11-Testing in Travale-Radiocondoli Field." Proceedings of the Larderello Workshop on Geothermal Resources Assessment and Reservoir Engineering (1977): 1-12.

The general objectives of well testing and well test analysis are discussed. Common procedures employed in the oil and gas industry and ground water hydrology are outlined and problem areas discussed.

Application of these techniques to geothermal conditions is described. It appears that standard techniques are useful in some cases; however, in general, the different nature of geothermal resources will require techniques be developed to handle boiling and nonisothermal flow and fracture dominated formation conductivity. It is pointed out that often the nature of the physical phenomena must be inferred from an analysis of the data. The difficulties of obtaining data in hostile geothermal environments is also outlined.

Averiev, V. V. "The Technique of Testing Geothermal Wells." Proceedings of the U.N. Conference on New Sources of Energy 2 (1964): 54-59.

Techniques of testing geothermal wells are discussed. The range of physical and chemical data that should be collected at static and flowing conditions in order that the power potential of the well and the downhole formation characteristics can be defined is identified. The dynamics of steam-water flow in geothermal wells and the hydrologic connection between wellhead and downhole conditions are also described.

Baba, K. and others. "A Study of the Reservoir at the Matsukawa Geatherma 1 Field." U.N. Symposium on the Development and Utilization of Geothermal Resources. Pisa, 22 September-1 October, 1970: 1440-7447.

Data from drilling and logging of wells at the Matsukawa Geothermal Field are compiled and used to assess probable permeable strata. Temperature variations in wells are also discussed in terms of permeability and fluid circulation. Formation permeability is estimated from well flow characteristics and pressure changes, and some assumptions are made concerning drainage areas. 
Balagna, John. "Geothermal Chemistry Activities at LASL, January - December 1975." Los Alamos Scientific Laboratory Report LA-6443-PR (1976).

The support geothermal chemistry work at LASL is described. The specific areas covered include: granite permeability studies, granitewater studies, and trace element work.

Permeability measurements, using a newly developed technique to allow measurements at elevated temperature and pressure (up to $200^{\circ} \mathrm{C}$ and $0.4 \mathrm{~Kb}$ confining pressure), show granite has a minimum in situ permeability of a few tenths of a microdarcy. In the granite-water studies felsic rocks were more reactive than crystallized mafic rocks but less reactive than glassy mafic rocks. Trace elements were examined as possible tracers for dissolution of minerals.

Banwel1,. C. J. "Geothermal Drillholes: Physical Investigations." Proceedings of the U.N. Conference on New Sources of Energy 2 (1964): 60-71.

This paper deals with the siting of investigation bores in a thermal area. Use of data in assessing the potential of the area for power production, for the siting of production bores, and for the subsequent monitoring of the behavior of the hydrothermal system during exploitation is also discussed. The siting procedures and interpretations are based on experience gained in exploration in the thermal area in the North Island of New Zealand, with particular reference to the Wairakei area, where large-scale development is now well advanced.

The siting of investigation bores using geophysical and geochemical surveys is discussed. The information that can be obtained from these bores is outlined and the subsequent siting, testing and monitoring of production wells is discussed.

Barelli, Antonio and others. "Build-up and Back-Pressure Tests on Italian Geothermal Wells." U.N. Symposium on the Development and Utilization of Geothermal Resources. San Francisco, 20-29 May, 1975: 1537-1546.

Build-up curves of some Italian geothermal fields (Larderello, Travale, Mt. Amiata) analyzed with oil-well test methods revea 1:

- Transmissivity $(K h)$ values ranging between 1 and 200 Darcy-meters. Transmissivity values obtained with different methods are consistent with one another.

- Generally negative skin effect coefficients.

- Well-bore storage coefficients which are usually higher than those calculated on the basis of well volume and fluid properties.

For most of the high productivity wells, back-pressure curves reveal the existence of a non-Darcy flow in the reservoir. The $K h$ values thus obtained are compatible with the results of build-up tests. 
Barelli, A. and others. "Horner Method Applied to Buildup Tests on Travale 22 We11." Workshop on Geothermal Reservoir Engineering. Stanford. (Stanford University Press, 1975): 101-112.

Production and buildup data from a well in the Travale geothermal field in Italy is analyzed using the Horner method. Extensive use of the superposition principle was necessary due to the considerable fluctuations in production history. Some qualitative observations were made based on the results. It appears the region of high permeability is located in a structural high which is surrounded by a lower permeability zone. Although not treated here the contribution to pressure buildup from a boiling zone at the boundary could not be excluded.

Barelli, A. and others. "An Interference Test in Alfina Geothermal Field (Northern Latiu, Italy)." Workshop on Geothermal Reservoir Engineering. Stanford. (Stanford University Press, 1975).

Production and interference data from wells in the Alfina geothermal reservoir are presented. Reservoir structure is described in terms of a free carbon dioxide bubble trapped beneath a cap rock. A water-saturated zone is present beneath the gas bubble. Standard pressure transient analys is is not appropriate since two separate phases are present and although water is produced the reservoir pressure is controlled by the gas cap. In addition the reservoir exhibits fracture conductivity rather than matrix permeability. However, pressure transient analysis was useful for indicating the presence of boundaries and obtaining preliminary estimates of diffusivity and transmissivity.

Bernard, William J. "Reservoir Mechanics of Geopressured Aquifers." First Geopressured Geothermal Energy Conference. Austin, Texas, 2-4 June 1975: 157-169.

This paper reviews established petroleum-reservoir engineering techniques of borehole pressure transient analys is as applied to geothermal geopressured systems. Single and multiple well tests are discussed in terms of aquifer geometry. Also the effects of dissolved natural gas, shale-water influx, and abnormally high rock compressibilities on aquifer behavior are outlined. It is concluded that dissolved natural gas has little effect on reservoir behavior whereas it is anticipated that rock compressibility has a significant effect on the energy deliverability. The role of shale-water influx as a reservoir drive mechanism was not clearly resolved.

Black, H. T. A Subsurface Study of the Mesa Geothermal Anomaly, Imperial Valley, Califormia (National Technical Information Service, Springfield, Illinois, 1975) (PB 247 082).

A conceptual model of the Mesa anomaly in Imperial Valley is inferred from a study of the geological characteristics and histories of five geothermal wells in the area. Also included are geophysical, geochemical and hydrological data. 
The coincidental resistivity, thermal, and gravity anomalies are connected with the presence of hot, saline water convecting in a hydrothermally altered fractured system. The system is apparently associated with the intersection of several near-vertical faults. Hot water rising from depth in the fault zone region spreads laterally below the $2500 \mathrm{ft}$ level merging with the regional northwestern ground water flow pattern.

Bodvarsson, Gunnar. "Thermoelastic Phenomena in Geothermal Systems. Second U.N. Symposirm on the Development and Use of Geothermal Resources. San Francisco, California, 20-29 May 1975: 903-907.

Thermoelastic effects of temperature changes in homogeneous rock and fracture systems with assumed Hookean elasticity behavior are studied. It is shown that the interpretation of surface deformation in terms of thermally induced subsurface volume strain is analogous to the interpretation of gravitational field data. It is suggested that response of ground surface can be used to monitor subsurface temperature changes and the progress of geothermal energy extraction. Moreover, changes in the temperature of flowing water result in fracture contractions or expansions which affect fracture fluid conductivity. These effects could be very significant in a narrow fracture system.

Celati, Romano and others. "Analys is of Water Levels and Reservoir Pressure Measurements in Geothermal Wells." Second U.N. Symposium on the Development and Use of Geothermal Resources. San Francisco, California, 20-29 May 1975: 1583-1590.

Downhole pressure and water level information is used to predict unknown formation pressures of the Larderello Geothermal Field. Newly drilled areas are compared geologically and hydrologically with Larderello in order to estimate likely formation conditions. Transition regions between water- and vapor-dominated systems are noted on the periphery of the heavily produced zone near outcrops of aquifer type rock.

Celati, R. and others. "An Attempt to Correlate Kh Distribution with Geological Structure of Larderel10 Geothermal Field." Workshop on Geothermal Reservoir Engineering. Stanford. (Stanford University Press, 1975): 37-41.

Transmissivity values obtained for 50 wells in the Larderello Geothermal Field are correlated with structural features established from geological and geophysical surveys. High transmissivity zones correlate with structural highs where compressive stresses have created tension cracks, fissures and cavities at the crest of anticlines. Permeability in these zones is thought to be mainly due to these fractures. It is concluded that tectonic action during the last phases of orogenes is has played a major role in determining the secondary (fracture) permeability of the Larderello system especially in formation contact zones. 
Chen, Bill. Geothermal Reservoir and Well Test Analysis: A Literature Survey. Report prepared for the National Science Foundation Applied to National Needs by the University of Hawaii, Hilo College (1974).

This survey deals with the various well testing techniques which can be used to estimate the formation characteristics of reservoirs. Techniques developed for the oil and gas industry are presented and shown to be applicable in many geothermal situations where the assumption of isothermal flow through isotropic material is valid. The basic fluid flow equations for radial isothermal flow through a porous formation are established and solutions of these equations for different boundary conditions are described. Specific topics which are discussed in some detail include interference testing, pressure drawdown and buildup, skin effect and wellbore storage, gas-liquid flows and fractured wells. In addition, a zero order lumped parameter material energy balance is presented which can be used to describe the thermodynamic performance of a geothermal reservoir under production. Twenty-two references are cited.

Chen, Bill H. and others. "Well Test Results from HGP-A." Geothermal Resources Council Transactions 2 (Part 1, 1978): 99-102.

Results of flow and pressure buildup tests are reported for the HGP-A geothermal well in Hawaii. It is concluded that the well is capable of producing two-phase fluid over a range of wellhead pressures from 50 to $237 \mathrm{psig}$ where the steam quality varies from $64 \%$ to $52 \%$. Moreover, the Kapoho Geothermal Reservoir appears to be tight with possible severe mud damage in the well.

Chen, H. K. "Experimental Steam-Water Relative Permeability Curves." Geothermal Resources Council Transactions 2 (Part 1, 1978): 103-104.

Steam and water relative permeabilities have been determined at various liquid water saturations using bench-scale model flow data.

The linear flow experiments were performed by allowing water to boil as it flows through cylindrical consolidated cores. The Hasslertype coreholder was located in a constant-temperature $\left(300+^{\circ} \mathrm{F}\right)$ a ir bath. Synthetic cement-consolidated sandcores were made with a thermowell and a glass-tube capacitance probe guide cast in place. The capacitance probe was used to determine the liquid water saturation along the length of the core.

The results suggest that relative permeability is a function of both liquid water saturation and temperature, and the practical irreducible water saturation appears to exceed $60 \%$ in the two-phase region. 
Delisle, George. Determination of Permeability of Granitic Rocks in GT-2 from Hydraulic Fracturing Data. Los Alamos Scientific Laboratory Report LA-6169-MS (1975).

This report presents a mathematical model which calculates the average permeability of rock next to a fracture. The model was successfully applied to data obtained from the creation of hydraulic fractures in the granitic section of two exploratory holes drilled by the Los Alamos Scientific Laboratory at the Fenton Hill Hot Dry Rock Site.

Facca, G. "Natural Steam Geology and Geochemistry." Proceedings of U.N. Conference on New Sources of Energy 2 (1964): 219-228.

The following important aspects of economical steam production are discussed: (a) source of heat, (b) ways of transferring the heat, (c) ways of accumulating the heat and supply of water. In the earth's crust only magmatic masses can be a source of heat having an adequate output. Three possible cases are defined: (1) shallow intrusion, (2) volcano-pluton, (3) volcanism in the strict sense. The convection of exogenous hot water rather than the flow of magmatic volatiles has been assumed to be the most probable mechanism of heat transfer. The presence of an impervious cap rock has been defined to be a necessary condition for the existence of a geothermal field. This impervious rock cap is capable of accumulating heat in the lower aquifer level.

Garg, S. K. "Pressure Transient Analys is for Two-Phase Geothermal Reservoirs." Geothermal Resources Council Transactions 2 (Part 1, 1978): 203-206.

Equations are presented for the pressure transient analysis of geothermal wells producing from a two-phase steam-water reservoir. The theory is tested by comparison with computer-generated simulations of typical drawdown history and is shown to produce realistic results for the kinematic mobility of an initially two-phase system and for the case of a boiling front propagating from the well. The method could be used to provide additional information regarding relative permeabilities etc., if the enthalpy of the discharge is known.

Garg, S. K. "Two-Phase Flow in Geopressured Geothermal Wells." Energy Conversion 18 (1978): 45-51.

The production characteristics of two-phase (free methane and liquid water with dissolved methane) geopressured geothermal wells are analyzed. The fluid flow in the aquifer is treated as single-phase (1iquid water with dissolved methane) unsteady radial Darcy flow; two-phase flow is assumed to occur only in the cased part of the production hole. The mathematical model allows for different gas and liquid velocities in the two-phase region. Sample calculations illustrate the effects on production of aquifer permeability and compressibility, the depth of the geopressured aquifer, the reservoir temperature, and the dissolved methane content of the aquifer fluids. 
Gulati, M. S. "Pressure and Temperature Buildup in Geothermal Wells." Workshop on Geothermal Reservoir Engineering. Stanford.

(Stanford University Press, 1975): 69-73.

The difficulties encountered when applying pressure-transient analysis techniques to hot water geothermal wells are outlined. Some drawdown data from a hot water well are used to illustrate these interpretive problems. Specific problems discussed include flashing in the reservoir with subsequent masking of the semi-log straight line, heat transfer toward the wellbore which masks drainage boundary effects, fracture flow and three-dimensional effects due to steam-water separation. It is concluded that improved techniques need to be developed which deal with these phenomena.

Hinrichs, Thomas C. "Niland Reservoir Monitoring and Evaluation Operating Program." Workshop on Geothermal Reservoir Engineering. Stanford. (Stanford University Press, 1975): 143-145.

A program of transient pressure testing, flowrate testing and chemical monitoring of produced and injected fluids in the Niland Geothermal Reservoir is described. The program is designed to provide reservoir performance data associated with the startup and operation of the thermal test loop.

Horne, R. N. "Steam/Water Relative Permeabilities from Production Data." Geothermal Resources Council Transactions 2 (Part 1, 1978): 291-293.

The steam-water relative permeability curves which were derived by Grant (1977) using well enthalpy and discharge histories are improved by eliminating some of the original assumptions. Specifically, relative steam-water discharges are back-calculated to the well-base, the effects of reservoir depletion are eliminated, and downhole fluid properties are calculated from the actual static well temperature rather than a field average. The relative permeability curves using data from three bores at Wairakei, New Zealand, are calculated and shown to be characteristic of flow in fractures.

Hsu, Y. C. Forced Oscilzations of the tos AZcamos Scientific Iaboratory's Dry Hot Rock Geothermal Reservoir. Los Alamos Scientific Laboratory Report LA-6170-MS.

Equations of motion are derived for the Los Alamos Scientific Laboratory's geothermal reservoir consisting of an elastic half-space (rock), with an open vertical pipe from the surface connected at depth with a vertical penny-shaped fracture. - This reservoir is subjected to seismic waves and/or blast-like impulses applied downhole.. The dynamic response of the water column, of the downhole internal net pressure, and of the fracture itself is studied. The natural frequency of the system can be experimentally determined and subsequently used to calculate the radius of the fracture. 
James, R. "Factors Controlling Borehole Performance." U.N. Symposizm on the Development and Utilization of Geothermal Resources. Pisa, 22 September - 1 October 1970: 1502-1515.

The discharge characteristics of geothermal boreholes are studied. Relationships are derived for the fall in borehole discharge with a drop in reservoir pressure and temperature and the effect of well diameter on discharge. For boreholes drawing from a source of pressurized water, the maximum wellhead pressure at which the well will maintain a discharge can be used to estimate the enthalpy of the formation fluid. From this the borehole characteristic curve can be estimated from discharge tests where flow rates are conveniently measured using a critical discharge meter.

James, Russe11. "Drawdown Test Results Differentiate Between Crack Flow and Porous Bed Permeability." U.N. Symposizm on the Development and Use of Geothermal Resources. San Francisco, 20-29 May 1975: 1693-1696.

Drawdown data from geothermal wells in El Tatio, Chile, and Cerro Prieto, Mexico, are used to demons trate that the gradient of the discharge versus pressure plot will indicate whether flow in the formation to the wellbore is through a crack or porous matrix. Estimates of crack width are also made and the effects of underreaming discussed.

It is proposed that pressure drawdown measurements can be used to determine whether a particular borehole is producing primarily from fractures or from a porous matrix.

Kunze, J. F. "The Experience of Getting Water Out of the Raft River Geothermal Wells." 17th International Rock Mechanics Symposium. Snowbird, Utah, 16-17 August 1976: 3B4-1 - 3B4-5.

The test drilling and reservoir evaluation program at the Raft River geothermal site is discussed. Well logs and cores indicate that production is mainly from fractures. Transmissivities determined from transient pressure test techniques reveal overall low rock permeabilities. Since production is from fractures which extend over very short horizons, the importance of drilling with a light fluid and the necessity of testing before casing the hole is stressed.

Kunze, Jay. F. "Raft River Geothermal Reservoir Engineering and Well Stimulation." Workshop on Geothermal Reservoir Engineering. Stanford. (Stanford University Press, 1975): 117-123.

The geothermal development program at Raft River is summarized. The production wells have been flow-tested to determine production horizons and thermal gradients have been measured at shut-in conditions. The laboratory determined permeability of core samples has been moderate. It is concluded that major reservoir permeability is due to fractures. 
It appears the decision to drill with water in the production zones has assisted the subsequent deliverability of the production wells.

The inferred structure of the reservoir determined from geophysical measurements has been correlated with the core, well $\log$ and drilling information.

McNabb, A., Grant, M., and Robinson, J. Permeability Estimates. Dept. of Scientific and Industrial Research Technical Publication No. 34 (Wellington, New Zealand, 1975).

The flow characteristics of wells in the Wairakei geothermal system indicate that production is locally through fractures rather than a permeable matrix. However, it is submitted that overall permeabilities can be defined on a macroscopic scale. A variety of methods are described for estimating both horizontal and vertical permeabilities. These include: (1) Pressure and temperature profiles measured prior to appreciable production with an estimate of the natural energy output of the field. (2) Vertical two-phase flow conditions which prevail in the top of the system in conjunction with the equations of mass and energy. (3) Temperature decreases as a result of boiling and subsequent mobility of a steam phase. (4) Pressure propagation between boreholes using estimates of compressibility for the elastic rock-water system. (5) Pressure drawdown in a hot cylindrical plume and recharge from cold margins. (6) Fissure permeability by analys is of pressure and temperature profiles and local temperature anomalies in wells. (7) Propagation of an elastic decompression wave giving an estimate of diffusivity. (8) Gross estimates of the area total heat flux of the system.

It is concluded that the Wairakei system exhibits an overall permeability of $7 \times 10^{-15} \mathrm{~m}^{2}$ with locally higher values along and normal to the direction of major faults. In addition, there is no evidence for the existence of either impermeable walls or a cap rock to the system.

Mann, Lloyd. "Well Log Analys is and Well Testing in the Heber Geothermal Field. " Workshop on Geothermal Reservoir Engineering. Stanford. (Stanford University Press, 1975): 74-76.

An approach commonly used in the oil industry is followed in evaluating the production potential of the Heber geothermal field. Well core, well $10 \mathrm{~g}$ and well test analyses were performed. Retrieved core resuits were correlated with available density logs to provide estimates of porosity and permeability with depth. Permeability from well buildup tests correlated favorably wi th the core- and log-determined values. It is concluded that the Heber reservoir could support 200 MW of generation. 
Meidav, Tsvi. "Utilization of Gravimetric Data for Estimation of Hydrothermal Reservoir Characteristics in the East Mesa Field, Imperial Valley, Cal ifornia." Workshop on Geothermal Resemoir Engineering. Stanford. (Stanford University Press, 1975): 52-61.

Small gravity anomalies in the East Mesa Geothermal Field are used to infer the general nature of convective heat, mass transfer and geochemical conditions of the field. It is submitted that small positive gravity anomalies are the result of chemical deposition due to cooling of a rising convective plume. The mass of precipitates is estimated and used to determine the quantity of water which has circulated through the system. This in turn is used to estimate the life of the system based on estimated permeabilities and mass velocities. The convective and conductive heat transfer is also estimated on the basis of the temperature drop needed to precipitate the chemicals and on the depth of the system.

Moench, A. F. and Herkelrath, W. N.. "The Effect of Vapor-Pressure Lowering Upon Pressure Drawdown and Buildup on Geothermal Steam Wel1s," Geothermal Resources Council Transactions 2 (Part 2, 1978): 465-468.

The equilibrium vapor pressure of liquid water in an unsaturated porous medium is less than the steam-table value. This phenomenon is incorporated into a finite-difference model for the production of steam in vapor-dominated geothermal reservoirs. In the model the steam is assumed to be produced by boiling, immobile liquid water in the vapordominated zone. Assuming radial symmetry, plots of pressure buildup and drawdown are obtained both with and without vapor-pressure-lowering. The results show that whereas pressure drawdown is virtually unchanged, the pressure buildup curve is significantly different when the vaporpressure lowering effect is included. The pressure plateau predicted by the model without vapor-pressure lowering is eliminated and the response is similar to that expected for noncondensable gas.

Morse, John G. and Thorson, Lewis D. "Reservoir Engineering Study of a Portion of the Salton Sea Geothermal Field." Geothermal Resources Council Transactions 2 (Part 2, 1978): 471-474.

Results are presented of a well testing program conducted in the Salton Sea Geothermal Field in the vicinity of the Geothermal Loop Experimental Facility. Flow parameters of interest in the study were the horizontal and vertical permeability, injectivity and the location of structural boundaries: Sensitive pressure measurement transducers were used to obtain the data from six wells, and a computer program using superposition of analytic solutions was developed to assist in analyzing the data. Initial results indicated that the reservoir has horizontal permeabilities of 100 to 500 md while vertical permeabilities across the shale layers are from 0.1 to 1.0 millidarcy. 
Narasimhan, T. N. and Witherspoon, P. A. "Initial Results of Reservoir Production Tests, Raft River Geothermal Project, Idaho." Workshop on Geothermal Reservoir Engineering. Stanford. (Stanford University Press, 1975): 124-125.

The results of the production and interference tests conducted on the geothermal wells RRGE 1 and RRGE 2 in Raft River Valley, Idaho, are presented. Three tests were conducted, two of them being short-duration production tests and one, a long-duration interference test. In addition to providing estimates on the permeability and storage parameters of the geothermal reservoir, the tests also indicated the possible existence of barrier boundaries. The data collected also indicated that the reservoir pressure varies systematically in response to earth tide effects. The results of the tests were analyzed using standard pressure transient analysis methodology and indicate that the geothermal reservoir is fairly extensive and significantly permeable and merits further exploration.

Piwinski, A. J. and Netherton, R. "Permeability of Kayenta Sandstone to Hypersaline Brine at 10.3 MPa Confining Pressure and Temperature to $90^{\circ} \mathrm{C} . "$ Third Stanford Geothermal Reservoir Engineering Workshop. Stanford. (Stanford University Press, 1977): 24-28.

Experiments were carried out on the flow of brines through sandstone core material at temperatures between $70^{\circ} \mathrm{C}$ and $90^{\circ} \mathrm{C}$ and confining pressures of $10.3 \mathrm{MPa}$ to evaluate changes in formation permeability and porosity at depth at the Salton Geothermal Field.

The data indicated that large permeability losses occurred in Kayenta sandstone (porosity, 20.7 $\pm 1.66 \%$ ) when unfiltered, untreated Magmamax brine and filtered, acidified Magmamax brine were the permeating fluids. In the former case, permeability decline was due to the accumulation of a thick. filter cake on the top face of the core sample which was composed of amorphous silica and iron. In the latter situation, loss of permeability was caused by the precipitation of amorphous silica and generation of large quantities of calcite particles from the dissolution of the matrix cement.

Ramey, Henry J. Jr. "Pressure Transient Analysis for Geothermal Wells." U.N. Symposium on the Development and Use of Geothermal Resources. San Francisco, 20-29 May 1975: 1749-1757.

A review of the history of pressure transient analys is and the application to geothermal wells is presented. Pressure drawdown and buildup, well interference and the effects of boundaries and fractures are discussed. Standard type curves are presented and their use outlined. Buildup data for steam wells in the geysers are presented and shown to be suitable for analysis using standard techniques. Use of the standard techniques in the presence of boiling and two-phase flow is cautioned. 
Ramey, Henry J. Jr. and others. Thermodynamic and Hydrodynamic Properties of Hydrothermal Systems. Stanford Geothermal Program TR-6

(Stanford University Press, 1974).

This paper outlines the various reservoir fluid and rock properties which are used to form the constitutive relations necessary to solve mathematical representations of reservoir models. Properties discussed include: thermal conductivity, thermal diffusivity, heat capacity, latent heat of phase change, permeability, wetability, relative permeability, porosity and thermodynamic equations of state for water, steam and brines. Methods for estimating these properties and various theoretical and empirical studies on the effects of pressure, temperature, and saturation are surveyed and briefly discussed.

Ramey, Henry J. Jr. "Wellbore Heat Transmission." Journal of Petroleum Technology 14 (1962): 127-135.

This paper presents an approximate solution to wellbore heattransmission. The solution permits estimation of the temperature of fluids, tubing and casing as a function of depth and time. The solution assumes that heat transfer in the wellbore is steady-state, while heat transfer to the earth will be unsteady radial conduction. Allowance is made for surface heat transfer coefficients for the case of gas flow in the wellbore. Comparisons of computed and field results are presented which establish the usefulness of the solution.

Rice, Larry F. "Pressure Drawdown and Buildup Analys is in Geothermal Reservoirs." Eleventh Intersociety Energy Conversion Engineering Conference. Stateline, Nevada, 12-17 September 1976: 798-801.

Transient pressure drawdown and buildup tests are used to determine permeability for one- and two-dimensional computer-generated reservoir behavior. In the single-phase problems, very good agreement with the classical theory was demonstrated. However, in the multiphase problems, the classical interpretation led to large errors in the predicted permeability data. These errors emphasize the need to establish a detailed reservoir simulator which will handle the often encountered boiling phenomenon and multiphase change which occurs in geothermal systems.

Roegiers, J. C. "Rock Characterization at a Geothermal Site." 17th International Symposizm on Rock Mechanics. Snowbird, Utah, 25-27 August 1976: 505-1 - 505-6.

Several geophysical logs were run in a 3-kilometer borehole that had been drilled for the purpose of extracting heat from a hot-dry granitic formation. Rock characterization tests were performed, both in the laboratory and field, to assist in the design of the hydrofracturing experiment and in improving drilling technology. Most of the properties determined from interpretation of the in situ geophysical measurements showed reasonably good agreement with the ones obtained under similar 
laboratory conditions. The minor discrepancies can be explained either by the presence of small open cracks in the rock core or by differences in the moisture environment. Greater discrepancies encountered in determining the porosity of the rock formation probably resulted from the unsuitability of empirical formulas derived from experience gained in testing soft porous rock masses.

Rumi, 0. "Some Observations About the Thermo-Fluid-Dynamic Behavior of the Steam in the Wells of Larderel1o and About the Best Exploitation Conditions." U.N. Symposium on the Development and Utilization of Geothermal Resources. Pisa, 22 September-1 October 1970: 698-703.

The conditions of flow in many wells have been investigated. Particular attention has been paid to the influence of the thermodynamic state of the vapor, of the friction factor of the casing tube, of the flow-rate, and of the well geometrical characteristics. From the results obtained, the conditions of best exploitation are examined and discussed. It appears that energy losses in many of the higher flow rate wells would be significantly reduced if the well diameter were larger. In some cases the wellbore controls the deliverability.

Sanya1, S. K. "Effect of Temperature on Petrophysical Properties of Reservoir Rocks." Society of Petroleum Engineers Paper No. 4898, DaZZas, (1974).

This paper reviews current knowledge of the effect of elevated temperature on pore volume, permeability, electrical resistivity and capillary pressure behavior of reservoir rocks. Changes in the properties of the rock matrix or the fluids and changes in the wetability of the rock-fluid system are examined in light of the similarities and contradictions in the published results, considering the basic petrophysical relationships and idealized pore geometries. It is concluded that the temperature sensitivity of rock-fluid wetability in explaining the observed temperature effects on petrophysical properties may have been over-emphasized in the literature, and in this regard consideration should be given to the change in fluid viscosity and rock matrix with temperature. Possible errors in well-log analys is and formation evaluation are pointed out which result from the common practice of neglecting temperature effects on some rock properties.

Schroeder, R. C. Reservoir Engineering Report for the Magma-SDG \&E Geothermal Experimental Site Near the Salton Sea, California. University of California Report UCRL-52094 (Lawrence Livermore Laboratory, 1976).

The structural and thermal features of the Salton Sea geothermal reservoir are outlined. The flow characteristics are given after analyses of drillstem tests and extended well tests. The field production, 
reserves and depletion are estimated, and the effect of fractures on flow and depletion are discussed.

The reservoir is believed to be separated into an "upper" and "lower" portion by a relatively thick and continuous shale layer. The upper reservoir is highly porous, with high permeability and productivity. The lower reservoir is at least twice as large as the upper but has much lower storativity and permeability in the rock matrix. The lower reservoir may be highly fractured, and its temperature and dissolved solids are greater than those of the upper reservoir. The proven reserves of heat in the upper reservoir are about $1 / 4 \mathrm{GW} \cdot \mathrm{yr}$ (in the fluid) and $1 / 3 \mathrm{GW} \cdot \mathrm{yr}$ (in the rock). In the lower reservoir the proven reserves are 5-3/4 GW.yr (fluid) and $17 \mathrm{GW} \cdot \mathrm{yr}$ (rock).

Stoker, Alan K. "Radon in Geothermal Reservoirs." U.N. Symposium on the Development and Use of Geothermal Resources. San Francisco, 20-29 May 1975: 1797-1803.

It is proposed that radon release from geothermal reservoirs may serve as a subsurface tracer for evaluating reservoir parameters such as porosity and fracture distribution. Several sampling and measurement techniques were evaluated to provide representative values for radon in hydrothermal systems. The effect of changing flow rate on radon concentration was theoretically predicted.

From preliminary drawdown and tracer measurement tests it was concluded that the use of radon appeared to be a promising reservoir engineering tool.

Tewhey, John D. and others. "Development of Injection Criteria for Geothermal Resources." Geothermal Resources Council Transactions 2 (Part 2, 1978): 649-652.

Experimental studies were conducted to evaluate injection of "spent" geothermal brine at the Salton Sea Geothermal Field. The subsurface medium was represented by membrane filters with pore diameters ranging from 0.4 to 10 microns and by sandstone core material. Changes in permeability with time were evaluated with respect to the following variables: (1) prefiltration of brine upstream from the core or membrane filter, (2) brine temperature $\left(20^{\circ} \mathrm{C}\right.$ to $\left.100^{\circ} \mathrm{C}\right)$, (3) brine $\mathrm{pH}$ [Owen, 1977] and (4) confining pressure.

No significant degradation in permeability was observed when silica saturated and moderately oversaturated solutions, prefiltered to $1 \mu \mathrm{m}$, were flowed through core samples. The core flushing results indicate that half-life estimates for injection wells obtained from $0.4 \mathrm{\mu m}$ membrane filters may be too conservative. The results with cores suggest that effluents with 1-2 ppm suspended solids are injectable at SSGF. 
Tolivia, Enrique M. "Evaluation of the Geothermal Potential of Cerro Prieto, Baja California (Mexico)." Second U.N. Symposium on the Development and Use of Geothermal Resources. San Francisco, 20-29 May 1975: 279-281.

Reservoir confirmatory studies carried out at Cerro Prieto, Mexico are described. Test wells were drilled outside the present zone of exploitation. Hydraulic tests on producing wells were conducted and indirect well $\mathrm{log}$ and direct laboratory measurement of rock porosity carried out. The potential of the field was evaluated by assuming a thermally and hydraulically isolated reservoir, and also by considering the known thermal and hydraulic recharge.

In the case of a model, isolated and limited by the presently exploited surface area, a minimal proven reserve of 33 years was calculated with $150 \mathrm{MW}$ installed. This is increased considerably if the area hydraulically affected by the present exploitation is included. These estimates did not include the effects of the known recharge and the estimates therefore are conservative.

Van Der Knaap, W. "Nonlinear Behavior of Elastic Porous Media." AIME Transactions 216 (1959): 179-187.

A theoretical and experimental analysis of the change in volume of a porous medium due to changes in external and internal pressures is presented. The uniqueness of this work is its capability to deduce the effect of large increments in the stresses directly as opposed to small increments in all previous works. Results from the study can find a direct application in material balance calculations and in problems of liquid flow through porous media. Since the elastic constants on the rock bulk material enter into equations for velocity of acoustic waves, the results are also of importance for calculating rates of wave propagation in these media.

Wainwright, D. K. "Subsurface and Output Measurements on Geothermal Bores in New Zealand." U.N. Symposium on the Development and Utilization of Geothermal Resources. Pisa, 22 september - 1 October 1970: 764-767.

The equipment and methods in current use on bores drilled for power production in New Zealand are described. Subsurface measurements of pressure and temperature are obtained by mechanical recording gauges and can be taken in four borehole conditions: (a) Cold water being pumped into the wellhead; (b) standing shut completely; (c) standing shut except for a single phase (water, steam or gas) bleeding from the welthead; (d) discharging normally with two-phase (water and steam) flow. The purposes of measurements in each of these conditions are discussed. Three different methods of bore output measurements are described: 
(a) measurement of separated steam and water flow; (b) measurement of mass and heat flows; (c) measurement of critical pressure and water flow rate at atmospheric pressure. The relative merits of these three methods are presented.

Yuen, P. "Preliminary Well Test Results from HGP-A." GeothermaZ Resources Council Transactions 1 (1977): 309-310.

Preliminary results from pressure transient analysis of the HGP-A geothermal well in Puna, Hawaii, indicates the presence of a large twoproduction layer reservoir of hot water in a tight basaltic formation. Well flow tests indicate that the well will support electric generation of 4 megawatts.

Yuhara, K. "Estimation of Hydrothermal Systems by Means of Wellhead Observations." U.N. Symposizm on the Development and Utilization of Geothermal Resources. Pisa, 22 September-1 October 1970: 1458-1462.

A model for two-phase boiling flow in a wellbore is presented. The model is used to predict boiling levels and downhole conditions from well lhead data. Reservoir drawdown is considered in deriving an equation for fluid flux into the borehole. Some applications of the method at the Otake and Beppu geothermal fields in Japan are given.

\subsection{Reservoir Modeling}

The mathematical basis for modeling of geothermal systems evolved in connection with analytical exercises associated with the physics of flow in porous media under adverse temperature gradients. Lapwood [1948] and Horton and Rogers [1945] studied the onset of convection in porous media using perturbation techniques to linearize the equations at the point of instability. The convection determining critical Rayleigh number was established for the ideal case of a constant property Boussinesq fluid. Many investigators have since shown that due to temperature sensitive variable viscosity and thermal expansivity, the critical Rayleigh number is considerably lower than the theoreticaliy predicted value of $4 \pi^{2}$ [Sorey,(1946); Combarnous, (1975); Kassoy,(1975); Straus,(1974); Morrison, (1949)].

Researchers were quick to realize that natural convection was a possible if not necessary process by which heat could move from deep in the lithosphere to accessible shallow horizons. The additional heat flux due to convective motions over conduction-dominated situations [Donaldson, 1970], oscillatory convection at high Rayleigh numbers [Horne, 1974], three-dimensional effects [Holst, 1972], and convection in sloping 
layers [Bories, 1973] are some basic convection-associated hydrogeologic phenomena which have been studied in detail. In a comprehensive review, Combarnous [1975] describes the basic theory of natural convection in porous media and outlines a number of techniques for treating specific problem areas.

The fundamental assumptions in almost all of the theoretical descriptions of natural convection include:

- Fluid and rock systems behave as continuums and volumeaveraged properties can be used.

- Thermal equilibrium exists between the fluid and rock.

- Overall thermal diffusivity can be defined.

- The Reynolds number is small.

- Darcys' law for flow in porous media is valid.

Mathematical justifications for these assumptions have been carried out in a number of studies [e.g., Assens, (1976); Pinder, (1975); Faust, (1976); and Kassoy, (1976)].

Due to the nonlinearity of the conservation equations, models for natural convection at above critical Rayleigh numbers generally must be solved by numerical methods. Two-dimensional models have been the most popular since they generally offer sufficient information for reasonable computational efforts. Both areal and cross sectional modeling approaches have been taken with the areal approach generally being more suitable for study of forced convection phenomena due to production from a reservoir and cross sectional studies being more informative in natural free convection studies.

Elder [1967] used finite difference techniques to establish generalized steady-state free convection patterns and studied the effects of various boundary conditions on the shape of the convection cells. In a subsequent study, Elder [1967] included transient terms to describe the motion of a hot blob of liquid released from the base of a porous slab and the development of convection cells when a slab is slowly heated. Other more geologically realistic geometries have been recently. investigated. Sorey [1976] and Kassoy [1976] analyzed convection arising from fractured or highly permeable "pipe" zones embedded in lower permeability surroundings. Cheng [1976] studied the boundary layer effects from convection induced around intrusive dykes. Cheng [1975] also derived an open-cycle natural convection cell to describe the heat transfer in island geothermal systems. Knapp [1976] and Isokari [1976] used established oil and gas procedures to develop two-dimensional finite difference models for water and gas production from geothermal geopressured reservoirs. The important reservoir drive mechanisms of formation compressibility, fluid expansion and water influx needed to be included in this case.

Integrated finite difference techniques have been developed to describe geothermal phenomena and have been particularly useful for 
incorporating structural discontinuities into quasi-three-dimensional models [Lasseter, 1975]. Three-dimensional finite difference models have also been developed by Coats [1977] who includes the possibilities of wells, fracture permeability and two-phase flow, and Kent [1978] who calibrates his model with some one-dimensional data for boiling flow in a core. The solution procedures (implicit by Coats and semi-implicit by Kent) were developed in connection with oil and gas reservoir modeling. Both models concentrate on simulating the production history of a finite body of hot fluid.

Finite element methods have been used successfully to study natural and forced convection. Faust [1975] compares finite element with finite difference techniques for single and two phase systems and concludes that finite element techniques are most suitable in the single phase cases. Mercer [1973] combines finite element and finite difference techniques in a two-dimensional areal simulator of a producing geothermal reservoir.

Mathematical models have been used diagnostically to interpret field data. For example, Donaldson [1970] proposes a method for estimating porosity and permeability at Wairakei on the basis of observed temperature and pressure gradients. Grant [1976] describes the severe drawdown at Broadlands on the basis of degassing of an initially high gas concentration system.

In recent years the emphasis has shifted from modeling of natural systems to simulations of systems which have been perturbed by the influence of production. In these cases, the effect of natural convection is not as dominant as that of reservoir sinks and sources through which fluid is being produced from or returned to the reservoir. Horne [1977] considered the effects of both natural convection and production in an axi-symmetric representation of Wairakei. The lowering of the water table and subsequent generation of a boiling surface was treated. Most of the forced convective models essentially trace the depletion of a finite mass of hot compressed fluids and are therefore eventually forced to consider the effects of boiling and nonisothermal flow. As a result, boiling and nonisothermal flow phenomena have been incorporated into many recent models [Mercer, (1975); Coats,(1977); Kent, (1978); Garg, (1978); Faust, (1976); Lasseter, (1975); Lipman,(1977); etc.]. The app 1icabifity of these models to real systems has been difficult to test because of the general lack of data on the nature of two-phase steam-water flow in porous media. In a rigorous study, Pritchett [1976] matched production history at Wairakei and identified localized areas of boiling within the reservoir. In addition to boiling phenomena, the secondary effects of net mass withdrawal and reservoir pressure drawdown such as subsidence [Brownel1, 1975] and induced cold water encroachment [Grant, 1976] have been recently studied.

Heat transfer from rock to fluid circulating in a fracture has received recent attention due to the increased interest in hot dry rock geothermal systems. Finite element methods are used by Bazant [1975] to describe fluid flow in the fractures. Bodvarsson [1977] considers 
laminated sheet-like conductors and postulates on the ranges of fracture separation for optimal energy withdrawal.

Experimental laboratory models or physical models have been used to evaluate mass and heat transfer processes which occur in geothermal systems. These models have potential uses for the calibration of mathematical models, for testing the validity of assumptions used in developing mathematical models (e.g., Darcy's law, relative permeability curves, etc.) and for evaluating mathematically intractable systems with anisotropic properties and complex geometries. The visual flow simulation reported by Elder [1976] was an early attempt at physical modeling with the emphasis on geothermal applications. Free convection plumes were generated in a two-dimensional Hele-Shaw cell and the effects of various discharge-recharge strategies on the temperature and velocity fields were noted. In another early study, Wooding [1957] simulated steady state free thermal convection in saturated permeable materials.

Physical models have been very useful in the study of forced convection and boiling in porous media. The linear flow models of Cady [1977] and Arihara [1974] delineated possible rock fluid interactions and heat transfer phenomena for nonisothermal flow in porous media. These linear model studies have been particularly useful for identifying the importance of capillarity, steam water relative permeability, deposition, irreducible water saturation, etc., in two-phase systems.

Factors affecting reservoir deliverability have also been studied with physical models. Hunsbedt [1975] studied a laboratory model fractured geothermal system in order to define the most efficient method of energy extraction. Schrock [1976] deduced governing dimensionless parameters for hydrotherma 7 systems and modeled a geotherma 1 energy extraction loop in order to identify geothermal well spacing and flow rates. Schrock submits that the general problem with physical modeling is that realistic experiments are difficult and cumbersome to set up and perform.

It is evident that many mathematical, numerical and physical techniques are available for developing quantitative models of geothermal systems. The transfer from studies of abstract systems to simulations of real geothermal reservoirs must now be accomplished in order to verify these techniques in the practical case. Unfortunately, few reservoirs at this stage have been produced to the extent that a performance history can be established and, as a result, little data is available with which to test the methodology.

From the preceding outline of geothermal modeling, it can be seen that a wide variety of techniques have been used to study an equally wide variety of observed geothermal phenomena. The following annotations are intended to identify the major objective of the work, to outline the mathematical or physical modeling technique used, and to identify the major conclusions. The reader is therefore directed to the annotations for more definitive summaries in his particular area of interest. 
Arihara, Norio. A Study of Non-Isothermal single- and Two-Phase Flow Through Consolidated Sandstones. Stanford Report SGP-TR-2 (Stanford University, 1974).

An investigation was made of nonisothermal single- and two-phase flow of a single component (water) in consolidated porous media. A physical model was built to simulate linear flow experiments through cylindrical consolidated cores. Both natural (Berea) and synthetic cement consolidated sand cores were used for hot fluid and cold water injection experiments. The steady-state overall heat transfer coefficient was measured and found to depend slightly on mass flow rate. The thermal efficiency of hot and cold water injection was found to depend on the heat injection rate. Results for cold water injection to hot porous medium indicated that lower injection rate, or smaller temperature differences between the surroundings and injected water, is advantageous for heat scavenging from the surroundings by cold water injection into the geothermal reservoirs. Boiling flow experiments showed that significant decreases in both temperaure and pressure occur within the two-phase, region. It was further noted that when heat transfer between the core and the surroundings was of a low level, then steady state two-phase non-isothermal flow was assumed to be isenthalpic. This study was the first nonisothermal boiling flow work in a consolidated sand pack, although previous work on thermal oil recovery by hot fluid injection presented similar information.

Assens, Guy E. "Derivation, by Averaging, of the Equations of Heat, Mass and Momentum Transfer in a Geothermal Reservoir." Second Workshop on Geothermal Reservoir Engineering. Stanford University, 1-3 December 1976: 268-289.

The equations of continuity, momentum, and heat transport are averaged from the level of the pore size to that of a "representative elementary volume" of porous medium using classical averaging techniques. The resulting equations are shown to be suitable for numerical computation when some assumptions concerning the solid matrix, fluid properties and the fluid-solid interactions are introduced. In addition, the heat balance equation, written in terms of enthalpy with the pressure work term neglected, is shown to be more suitable for computation than when expressed in terms of internal energy.

Atherton, Robert W. "The Use of General Sensitivity Theory to Analyze the Geothermal Reservoir Model's Sensitivity to the Permeability Functions." Workshop on Geothermal Reservoir Engineering. Stanford. (Stanford University Press, 1975).

This paper outlines the use of general sensitivity theory for the study of the effects of large parameter uncertainty on geothermal reservoir simulators. The method is illustrated by application to two problems: flow in anisotropic porous media and a nonlinear (convective diffusion) flow problem. 
Atkinson, Paul G. Mathematical Modeling of a Single-Phase Non-Isothermal Fluid Flow Through Porous Media. Ph.D. dissertation, Stanford University (1976).

Experimental results for single-phase non-isothermal fluid flow through a linear core containing porous media were modeled mathematically. Analytical solutions of the partial differential wave and parabolic equations derived using different simplifying assumptions revealed that heat transport through the walls of the core is significant and should be considered when designing experiments. In addition, a method was developed for determining the permeability of a porous medium based on the propagation of isotherms under non-isothermal flow conditions.

Austin, Arthur L. and others. LLL Geothermal Energy Program Status Report, January 1976 - January 1977. Lawrence Livermore Laboratory Report UCRL-50046-76 (National Technical Information Service, Springfield, Illinois, 1977).

The reservoir engineering program in support of the Salton Sea Geotherma 1 Development is discussed. The following general topics are outlined: (1) interpretation of heat and mass transport processes on the bas is of borehole temperature gradients; (2) estimation of reservoir compaction and storability on the basis of borehole resistivity logs; (3) heat and mass transport for various production-injection strategies and arbitrary well arrays; (4) thermal depletion of combined fracture and pore permeability reservoirs; (5) thermal history of injected fluids in relation to the injected fluid flow field; (6) pressure transient analysis for production; and (7) injection wells and experimental studies of deposition of salts in porous media.

Barelli, A. and others. "Progress Report on a Mathematical Model of a Parallelepiped Reservoir with No Penetrating Wellbore and Mixed Boundary Conditions." Workshop on Geothermaz Reservoir Engineering. Stanford. (Stanford University Press, 1976): 242-246.

This progress report describes the development of a mathematical model for simulating unsteady flow in geothermal reservoirs containing a single phase fluid. The model will be used to predict the performance of the Travale geothermal field in Italy. The reservoir is assumed homogeneous, isotropic and parallelepiped in shape with no flux boundary conditions. Production is assumed to occur through a non-penetrating well although other well conditions can be incorporated. Comparison of the results with standard oil field pressure transient techniques showed them to be inappropriate for models of this geometry.

Bazant, Z. P. "Finite Element Solution of Geothermal Energy Extraction." Workshop on Geothermal Reservoir Engineering. Stanford. (Stanford University Press, 1975): 232-239.

The initiation and extension of a crack created by hydraulic fracture in hot dry rock and the circulation of water through the crack 
is modeled using finite element techniques. The field equations for fluid flow are written in two dimensions with an assumed velocity profile across the crack. The Galerkin technique is used to obtain the finite element equations. The development of a two-dimensional finite element program for the rock is outlined. Since the computer time requirement for the finite element solutions is large, some simple analytical results were obtained in order to better understand the physical processes occurring within the system. These include: crack extension, heat extension in steady-state fluid flow, and the initiation of secondary cracks.

Bodvarsson, Gunnar. "Fracture Flow in Geothermal Reservoirs." Workshop on Geothermal Reservoir Engineering. Stanford. (Stanford University Press, 1975): 45-49.

Equations are derived describing the flow of water and steam through a fracture to the borehole. For formations which exhibit a high density of fractures, the flowrate through each will be so small that laminar flow conditions will prevail and Darcy's law can be used to estimate the bulk permeability. Various reservoir engineering techniques are described which are used in Iceland to predict reservoir parameters and production behavior. Stimulation of reservoirs is discussed in terms of reducing the bottom hole pressure, injection, and fracturing.

Bodvarsson, G. "Geothermal Resource Energetics." Geothermics 3 (1974): 83-92.

The theory of geothermal heat extraction is discussed on the basis of the following three simple idealized models: (1) single fracture flow; (2) intergranular flow; and (3) intergranular vaporization models. Theoretical expressions for the total recoverable specific resource energy per unit area or unit volume are given. The fracture flow model assumes heat is extracted solely from the fracture walls by conduction. The intergranular flow model assumes heat is transported by convection only, and the vaporization model considers only the latent heat released by boiling. The results are illustrated by a number of curves which can be used to assess the potential of individual geothermal resources.

Bodvarsson, Gunnar. "On the Temperature of Water Flowing Through Fractures." Joumal of Geophysical Research 74 (1969): 1987-1992.

Equations are given governing the transport of heat to a fluid flowing in a fracture in hot rocks. Two-dimensional analytical solutions are derived for the case of constant mass flow with fluid entry temperatures being a sinusoidal function of time. The rock is assumed to be a homogeneous and isotropic conductor. Approximate solutions are derived for the case in which the temperature at the intake is an arbitrary function of time. The case involving weak oscillations in the mass flow is treated on the basis of a perturbation method. Finally, some of the solutions derived are extended to the case of a laminated solid where the fluid flows between the laminas and thermal interference occurs. 
Bodvarsson, Gunnar. A Secondary Recovery Method for the Extraction of Geothermal Energy. (Oregon State University, Corvallis, 1977).

Computer programs were written to carry out numerical modeling of the heat extraction by sheet-1ike conductors (e.g., cracks in rock) where the fluid flow is a function of time. The effects of flow channeling were studied and the numerical models of convective flows were developed. The fundamentals of the theory of fluid injection into cracks and the resulting crack propagation were derived and streamlined for application to simple cases. New results on minimum contact areas and borehole flows were obtained.

It is concluded that the contact areas required for economic operation of secondary recovery methods are not excessive and are within the possibilities of present-day technology. However, due to a number of intangible factors such as the possibilities for severe flow channeling, fluid losses, etc., it is suggested a series of field experiments on natural conductors be carried out.

Bories, S. A. "Natural Convection in a Sloping Porous Layer." Journal of Fluid Mechanics 57 (1973): 63-79.

This paper describes an experimental and theoretical study of thermal convection in a sloping porous layer. The saturated layer is bounded by two parallel impermeable planes maintained at different temperatures. Results were obtained for Rayleigh numbers between 0-800 and slopes from $0-90^{\circ}$. Several types of flows were observed: a unicellular movement at low Rayleigh numbers and a juxtaposition of longitudinal coils or of polyhedral cells at Rayleigh numbers above a critical transition value.

A theoretical analysis was made by using the standard bases of the linear theory of stability and by taking into account some assumptions suggested by experimental observations. For flow in longitudinal coils or with polyhedral cells, the average heat transfer depends mainly on the filtration Rayleigh number and on the slope of the layer. For both the transition criterion and the heat transfer, a good fit was observed between the experimental and theoretical results.

Bories, Serge. "Fundamental Study of Changing of Phase in Porous Materials." Workshop on Geothermal Reservoir Engineering. Stanford. (Stanford University Press, 1975): 247-248.

This paper outlines a fundamental research program focused on the phenomenon of boiling in porous media. The assumption of equilibrium temperatures between the fluid and matrix is unrealistic when flow is largely through fissured media with large block dimensions. To investigate this nonequilibrium condition, the rock-fluid heat transfer process is being studied experimentally for a range of physical parameters including porosity, grain size and boundary conditions. A one-dimensional numerical model is being developed for comparison with the experimental results. 
Bredehoeft, J. D. and Papadopulos, S. "Rates of Vertical Groundwater Movement Estimated from the Earth's Thermal Profile." Water Resources Research 1 (1965): 325-328.

An analytical solution is developed describing vertical steady flow of groundwater and heat through an isotropic, homogeneous, and fully saturated semicontaining layer. A type-curve method is presented for estimating groundwater velocities from temperature data. Application of the method to detection of very small groundwater velocities through low permeability aquitards is demonstrated.

Brigham, William E. "Heat and Fluid Flow Experiments to Measure Geothermal Reservoir Physical Parameters." Workshop on Geothermal Reservoir Engineering. Stanford. (Stanford University Press, 1975).

Experimental model tests designed to study the heat and mass transport processes and physical parameters relevant to geothermal systems are discussed. The design and operation of a linear core flow model is outlined. This model has been used to investigate the various phenomena associated with non-isothermal boiling and two-phase flow in porous media. Particular topics have included a study of the dependence of absolute permeability on temperature and the establishment of steam/water relative permeability curves. The model has also been used to provide one-dimensional data on reservoir drainage which has been useful for calibrating mathematical models of geothermal systems.

Browne11, D. N. and Garg, S. K. "Computer Simulation of Geothermal Reservoirs." Society of Petroleum Engineers Paper No. 5381, Dallas, (1975).

General balance laws and constitutive relations are developed for convective hydrothermal reservoirs. Three levels of sophistication are treated. In the "passive" system, the rock matrix is assumed to be rigid and the system of equations reduces to a description of fluid convection and fluid-rock conduction. An order of magnitude analys is of the equations shows that inertia, thermal diffusivity, and pressure work terms can be neglected for hydrothermal systems. Fluid motion is characterized by the dimensionless Rayleigh number. In the quasi-active model the rock thermal properties are assumed functions of temperature, position and local pore pressure. Using this model some fluid-rock interactions can be taken into account. The quasi-active model can be expanded to the fully interactive case by supplying the constitutive relations for the rock matrix. Treatment of geologic stress-strain behavior of the material allows the highly nonlinear and history-dependent constitution behavior of rocks to be taken into account. 
Cady, C. V., Bilhartz, H. L., and Ramey, H. J. Jr. "Model Studies of Geothermal Steam Production." Water-1972 AIChE Symposizom Series. (American Institute of Chemical Engineers, New York, 1972): 445-452.

A physical model of a geothermal steam reservoir consisting of a 3-inch cylinder packed with sand which exhibited a permeability of 4.5 darcys and a porosity of $3.6 \%$ of bulk volume was operated over a range of conditions from a compressed liquid to boiling liquid to single-phase steam reservoir. No significant lowering of vapor pressure was observed in the unconsolidated sand model. Existence of dry steam zones and two-phase boiling zones were observed. Calculations using a materialenergy balance equation agreed with the observed production.

Celati, R. and others. "Study of Effect of Reinjection with a Mathematical Mode1." Proceedings of the Larderello Workshop on Geothermal Resource Assessment and Reservoir Engineering (1977): 308-326.

This interim report discusses the effects of condensate injection in vapor-dominated geothermal reservoirs. Specifically, data are presented from injection and drilling fluid loss in the Italian vapordominated reservoirs. A two-dimensional model which uses an integrated finite difference technique to solve the conservation equations is being used to study the effects of injection into the Castelnuovo and Serrazzano geothermal fields. The physical properties and boundary conditions are being optimized by matching with the past production performance of the reservoir.

Chen, Hsiu-Kuo. Measurement of Water Content in Porous Media Under Geothermal Fluid Flow Conditions. Ph.D. dissertation, Stanford University (1977).

Water saturation in a core through which two-phase boiling water is flowing is measured with a capacitance probe. The results are used to calculate relative permeabilities to water and steam. Deposition of salt is also studied by allowing a core initially saturated with a 1200 ppm hot sodium chloride solution to be depleted by allowing the brine to boil. Although salt deposition increased toward the point of fluid extraction, the absolute permeability remained unaffected when determined by flowing nitrogen gas through the core.

Chen, Jay C. and Conel, James E. "Finite-Element Solutions for Geothermal Systems." Joumal of Energy 1 (1977): 364-369.

Using vector potential and scalar potential, the governing equations for a single component and single phase geothermal system are formulated. By assuming an initial temperature field, the fluid velocity can be determined which, in turn, is used to calculate the convective heat transfer. The energy equation then is solved by considering convected heat as a distributed source. Using the resulting temperature to compute 
new source terms, the final results are obtained by iterations of the procedure. Finite-element methods are proposed for modeling realistic geothermal systems; the advantages of such methods are discussed. The developed methodology is then applied to a sample problem. Favorable agreements are obtained by comparisons to the previous study.

Cheng, Ping and Lau, K. H. "The Effect of Steady Withdrawal of Fluid in Confined Geothermal Reservoirs." Second U.N. Symposium on the Development and Use of Geothermal Resources. San Francisco, 20-29 May 1975: 1591-1598.

The problem of combined free and forced convection in geothermal reservoirs resulting from steady withdrawal of fluid is investigated numerically. The aquifer is confined by caprock on the top, heated by an impermeable surface at the bottom, and is recharged continuously from the ocean. The governing steady state quasi-linear partial differential equations in terms of pressure and temperature are approximated by finite difference equations which are solved numerically by iteration. Computations were carried out for both cylindrical and rectangular reservoirs for a selected set of parameters. It is found that the withdrawal rates and the location of the withdrawal site have a significant effect on heat transfer and fluid flow characteristics in a geothermal reservoir.

Cheng, Ping. "Heat and Mass Transfer in Liquid Dominated Geotherma 1 Reservoirs." Letters in Beat and Mass Transfer 3 (1976): 81-88.

Studies of heat and mass transfer processes in water-dominated hydrothermal systems are reviewed. The studies have been directed toward the analysis of free convection and production-injection processes in island hydrothermal systems. Thermofluid mechanics peculiar to these systems, such as freshwater-seawater interaction, open convective circulation patterns, water table uplift, and temperature reversals on aquifer margins, are explained with free convective models which use perturbation solution techniques near the critical Rayleigh number and numerical techniques at high Rayleigh numbers. Studies of the boundary layer effects of various shaped near-surface volcanic intrusives in high Rayleigh number flows are also reviewed. It is shown that these systems can be modeled using similarity theory.

Cheng, Ping. "Numerical and Analytical Studies on Heat and Mass Transfer in Volcanic Island Geothermal Reservoirs." Workshop on Geothermal Reservoir Engineering. Stanford. (Stanford University Press, 1975): 219-224.

The progress made on the theoretical study of heat and mass transfer in a volcanic island geothermal reservoir is reviewed. Free convection in an island aquifer, confined by caprock at the top and heated by bedrock from below, was studied using a numerical model. The results show that a major seawater convective cell is formed. In addition, smaller closed 
cells can be formed in the center of the system if the heating surface is sufficiently large. Various thermal gradient phenomena observed in the Hawaiian geothermal system are explained by the model.

Cheng, Ping. "Numerical Modeling of Hawaiian Geothermal Resources." Geothermics 2 (1973): 90-93.

Numerical modeling of island geothermal systems is discussed. Convection at low Rayleigh numbers was modeled where the governing nonlinear partial differential equations were approximated using a linear. perturbation method. The effects of the geothermal resource on the movement of seawater and upwelling of the water table were studied using this method. For high Rayleigh number conditions, the governing equations are solved using finite difference approximations. The effect of various boundary conditions and fluid withdrawal on the isothermal contours is presented.

Cheng, Ping. "Numerical Solutions for Steady Free Convection in Island Geothermal Systems." Future Energy Production Systems: Heat and Mass Transfer Processes II (1976): 429-448.

Steady free convection in an is land aquifer, confined by caprock at the top and heated by an impermeable surface below, is considered. The governing nonlinear partial differential equations are approximated by a set of finite difference equations, which are solved numerically by iteration. Factors which are analyzed include: the effects of thermal conditions at the caprock, the geometry of the reservoir, the variation of Rayleigh number, the length of the heating surface and of a magmatic intrusion on fluid flow, and heat transfer characteristics in island geothermal reservoirs.

Cheng, Ping. Numerical solution for Transient Heating and Withdrowal. of Fluid in a Liquid-Dominated Geothermal Reservoir. (University of Hawaii, Honolulu, 1976).

The transient responses in a liquid-dominated geothermal reservoir resulting from the sudden heating and withdrawal of fluids are studied numerically using finite difference techniques. A two-dimensional rectangular reservoir confined by caprock at the top, heated by bedrock from below, and recharged continuously from the vertical boundaries is studied. The governing equations are combined and reduced to two nonlinear partial differential equations in terms of dimensionless temperature and pressure. It is found that for an aquifer, $5000 \mathrm{ft}$ in depth and $20,000 \mathrm{ft}$ in width and with a convection/conduction ratio of 4000, steady-state conditions are reached at approximately 7000 years after the sudden heating from below. Oscillatory convection begins noticeably when the ratio of convection to conduction exceeds 4000 , the period of oscillation decreasing as this ratio increases. The contraction of isotherms in the reservoir resulting from the withdrawal of fluids from point sink and line sink are shown. 
Cheng, Ping and Lau, K. H. "Steady State Free Convection in an Unconfined Geothermal Reservoir." Journal of Geophysical Research 79 (1974): 4425-4437.

The problem of steady state free convection in an unconfined aquifer bounded by ocean on the sides with geothermal heating from below is investigated. The governing nonlinear partial differential equations with nonlinear boundary conditions are approximated by a set of linear subproblems on the basis of perturbation method. The equations for the zero- and first-order approximations are of the elliptic type and are solved numerically by the finite difference method. Numerical results, accurate to the first-order approximations, are obtained for temperature, pressure, and stream function as well as for the shape of the water table. The influence of the location and the size of the heat source on heat transfer and fluid flow characteristics including the movement of seawater, upwelling of the fresh water table, and temperature and pressure distributions in a rectangular geothermal aquifer are discussed.

Chicoine, S. D., Strobel, C. J., and Ramey, H. J. Jr. "Vapor Pressure Lowering in Two-Phase Geothermal Systems." Society of Petroleum Engineers Paper No. 6767. Dallas. (1977)

This study concerns the construction and operation of a laboratory model of a non-recharged (pressure depletion) two-phase geothermal reservoir. The primary emphas is is directed toward the significance of vapor pressure lowering phenomena caused by interfacial tension in a consolidated porous medium. Preliminary runs were made which indicated vapor pressure lowering effects as large as $15 \mathrm{psia}$ in a consolidated porous medium at geothermal system temperatures of $220^{\circ} \mathrm{F}$ to $290^{\circ} \mathrm{F}$. Model construction and operating details are given.

Coats, K. H. "Geothermal Reservoir Modeling." Society of Petrolevm Engineers Paper No. 6892. Dallas. (1977).

This paper describes an implicit, three-dimensional numerical model of a geothermal reservoir. The implicit method assures time step stability of the finite difference formulation. Illustrative applications of the method demonstrate the tolerance of the model in handling grid rock saturation changes of $80-100 \%$ and throughput ratios of $10^{8}$. Applications include single and two phase single-well behavior, fractured matrix reservoir behavior, well test interpretation, and extraction of energy from fractured hot rock.

Simulation of a single-phase flow pressure drawdown test in a tight formation containing horizontal fractures reveal an upward curvature trend of the drawdown versus $10 \mathrm{~g}$ time plot. Simulation of fractured hot rock systems show that significant increases in energy extraction could be effected with increased fracture surface areas. An analytical derivation of well deliverability reduction due to boiling near a wellbore is also presented. 
Coats, K. H. and others. "Three-Dimensional Simulation of Steamflooding." AIME Transactions 257 (1974): 573-592.

A three-dimensional model for numerical simulation of steam injection processes is presented. Three-phase flow of water, oil and steam, and heat flow in the reservoir and overburden is described. Mass and energy balances are simultaneously solved which eliminates the need for iterating on the mass transfer (condensation) term.

In order to check the accuracy and assumptions in the numerical model, an experimental model of the system was developed. Laboratory data are reported for steamfloods of $5780-\mathrm{cp}$ 0il in a 1/4 fine-spot sand pack exhibiting three-dimensional flow effects.

A need to account for effects of temperature on relative permeability became evident upon comparison of model results. Model calculations indicated that steamflood pressure level strongly affects oil recovery - calculated oil recovery increases with decreasing pressure level.

Cody, Gilbert. Model Studies of Geothermal Fluid Production. Ph.D. dissertation, Stanford University (1969).

The work describes experiments conducted to study the behavior of geothermal fluid systems. A model geothermal fluid reservoir was constructed of stainless steel tube packed with fine, unconsolidated sand. The sand pack was saturated with distilled water and maintained in a closed system at a temperature of $400^{\circ} \mathrm{F}$ and 315 psia pressure. Fluids (steam and water) were produced from the system by vaporization and expansion until the system pressure dropped to $50 \mathrm{psia}$.

The significance of vapor-pressure lowering phenomena in a porous system was the principal objective of the investigation. The produced data show that vapor pressure lowering can cause significant changes in material-energy balance. In addition, vapor pressure lowering may be responsible for liquid remaining in the rock after complete pressure depletion of the system. The measured mass produced in the experiments was checked with a material-energy balance model developed elsewhere and was found in good agreement. Vapor pressure lowering was not significant in these experiments. It is noted that large temperature differences may exist in two-phase geothermal systems, which may make the application of the material-energy balance more unpredictable.

Combarnous, Michel A. and Bia, Paul. "Combined Free and Forced Convection in a Porous Media." Society of Petroleum Engineers Journal 11 (1971): 399-405.

Temperature distribution and heat transfer resulting from convection was determined. Experiments were performed to study thermal convection in a horizontal porous layer bounded by isothermal planes with and without a mean flow of the saturating, fluid phase. A distinction was made between the natural or free convection occurring in a closed volume 
and the mixed or combined free and forced convection arising when there is mean flow of the fluid phase.

Theoretical criterion for the onset of convection (Rayleigh number $\mathrm{N}_{\mathrm{Ra}}>4 \pi^{2}$ ) was verified. Depending on the experimental conditions, a regular pattern of convective cells was observed which may have been motionless or moving for low values of $\mathrm{N}_{\mathrm{Ra}}(<260)$. For $\mathrm{N}_{\mathrm{Ra}}$ values higher than 260, another convective state was found that was mainly unstable.

To confirm the experimental results for the heat transfer and size of convective cells, a numerical model was developed.

Combarnous, Michel A. and Bories, S. A. "Hydrothermal Convection in Saturated Porous Media." Advances in Hydroscience 10 (1975): 231-307.

Single phase hydrothermal convection in porous media is studied in detail. Basic physical principles are discussed and methods are outlined for estimating the effective thermal conductivity of the solid fluid medium. The basic field equations are presented, and analytical and numerical techniques of solving the equations are discussed. The phenomena of cellular convection and instability are studied for systems of various geometry and orientation. Experimental methods are also considered. The Hele-Shaw cell is discussed in detail and experimentally observed phenomena are interpreted theoretically. Visualization of convection phenomena is shown to be useful for establishing the effects of boundaries and cell geometry on convective motions.

Numerical techniques for solving more complicated convection problems are described and combined free and forced convection problems are considered. Finally, more complicated convection problems are considered including convection in anisotropic porous media, thermohaline convection (compressibility important) and convection in nonuniformly heated layers.

Donaldson, I. G. and Grant, M. A. "An Estimate of the Resource Potential of New Zealand Geothermal Fields for Power Generation." Proceedings of the Larderello Workshop on Geothermal Resource Assessment and Reservoir Engineering (1977): 413-427.

The basic similarity between most of the New Zealand geothermal fields suggests that the exploited fields of Wairakei and Broadlands can be used as indicators of the potential of other fields. Assuming adequate permeability will be obtained in fields yet to be tested, the two parameters controlling this potential are areal extent (as defined by resistivity survey) and temperature at depth. As most field temperatures are bracketed by Wairakei $\left(270^{\circ} \mathrm{C}\right.$ maximum) and Broadlands $\left(300^{\circ} \mathrm{C}\right.$ maximum), field potential per unit area should also be bracketed by the field potentials per unit area of these two fields, i.e., Wairakei at 10-11 MWe $/ \mathrm{km}^{2}$ and Broadlands at $13-14 \mathrm{MWe} / \mathrm{km}^{2}$. Totals for all fields of 500 MWe proven, 900 MWe inferred, and 1100 MWe speculative suggest that New Zealand has some 1400-2500 MWe available from its geothermal resources. 
Donaldson, I.G. "The Estimation of Subsurface Flows and Permeabilities from Temperature and Pressure Data." U.N. Symposizm on the Development and Utilization of Geothermal Resources. Pisa, Italy, 22 September -1 October 1970: 677-683.

Pressures and temperature profiles measured in drillholes prior to discharge are analyzed to give some estimate of the pressure and temperature distribution in the upper region of an undisturbed geothermal field. Substitution of this information into the difference form of the primitive equations of motion of the fluid and heat system then leads to a set of equations with the vertical and horizontal permeabilities and the three components of mass flow as unknowns. These equations may be solved under suitable boundary conditions, and flow and permeability patterns can be estimated. In its present form the approach is only suitable for water systems; it cannot be applied if steam or gas is present.

Donaldson, Ian G. "The Flow of Steam Water Mixtures Through Permeable Beds: A Simple Simulation of a Natural Undisturbed Hydrothermal Region." New Zealand Journal of Science 11 (1968): 3-23.

The one-dimensional steady-state flow of a boiling liquid upwards through permeable material is studied, and the relationship between the temperature and flow characteristics of the system with respect to depth is established for flow through porous media over a range of permeabilities. The boiling systems appear to be controlled almost entirely by the ratio of the mass flow rate to the permeability. It is found, for low values of this ratio, that the depth over which boiling will occur may be as great as $\mathbf{5 5 0}$ meters but that this decreases as this ratio increases. The level at which boiling commences is marked by an imediate change to a $10 \%$ (by volume) steam content. Other sudden changes of this type will occur in vertical flow systems at any interface at which the permeability changes.

Donaldson, I. G. "Simulation of Geothermal Systems with a Simple Convective Mode1." U.N. Symposizm on the Development and Utilization of Geothermal Resources. Pisa, 22 September-1 October 1970: 649-654.

A convective hydrothermal system is conceptualized as two vertical permeable channels interconnected at the bottom. One of the channels acts as the system fluid reservoir while the other is the heated channel that feeds the hot springs and fumaroles that define the geotherma 1 field. The dimensions of the heated channel, its permeability, the temperature at depth (or the heat flux) and the resistance of the connecting strata are found to be significant factors in determining the form of the geothermal field. Some typical fields are briefly discussed with the aid of the model. 
Donaldson, I. G. "Temperature Gradients in the Upper Layers of the Earth's Crust Due to Convective Water Flows." Journal of Geophysical Research 67 (1962): 3449-3459.

The temperature and flow patterns that would be set up in a single, permeable layer bounded above and below by impervious conducting sheets and in a two-layer system consisting of an upper saturated permeable layer and an underlying layer of impermeable basement rock are determined approximately for planar water flows of convective type. Conservation equations for single phase flow in an isotropic porous media are written in terms of the stream function and solved numerically using a finite difference approximation. For the single-layer system only simple circulatory flows are considered, but for the two-layer system two types of flow are treated: purely circulatory flows, and flows incorporating a closed circulation of underground water combined with a circulation in which water enters the ground surface at some places and rises to be lost as surface runoff in other places. Simple circulatory flows can cause the surface heat flux to differ by a factor of 9 between regions of upward and downward flow. This surface heat flux difference is more extreme when discharge and recharge are included.

Dudarev, A. N. "Endoclave, a New Device for the Study of Heat and Mass Transfer by Simulation of Geologic Bodies and Processes Under Dynamic Conditions." U.N. Symposium on the Development and Utilization of Geothermal Resources. Pisa, 22 September - 1 October 1970: 1275-1279.

An experimental device is described which can be used to simulate the dynamic heat and mass transfer processes which occur in geothermal systems. General design and operational principles are outlined and results of tests on some typical reservoir rock are described.

Elder, J. W. "Numerical Experiments with Free Convection in a Vertical Slot." Joumal of Fluid.Mechanics 24 (1960): 823-843.

Numerical solutions of the equations of steady free convection in a vertical slot have been obtained by a procedure which relies solely on an interactive solution of Poisson's equation. With a mesh spacing of $1 / 40$ the solutions are stable up to a Rayleigh number of order $10^{5}$. The numerical experiments are comparable to results from laboratory experiments. The growth of boundary layers and the uniform vertical temperature gradient are well described. It is tentatively suggested that certain unstable motions found prior to the divergence of the numerical solution are comparable to the secondary flows found in experimental models. 
Elder, J. W. "Steady Free Convection in a Porous Medium Heated from Below." Journal of Fluid Mechanics 27 (1967): 29-48.

This paper describes an experimental and numerical study of steady free convection in a porous medium. The steady-state two-dimensional equations of heat and mass transport are solved using a finite difference approximation and compared with experimental results obtained from a Hele-Shaw cell. The effects of boundaries on the distribution and size of convection cells are investigated and disturbances to the cells as a result of mass discharge are noted.

The onset of free convection in porous media is compared with analogous Benard-Rayleigh flows for viscous fluids. The heat transferred across the layer is found to be proportional to the square of the temperature difference across the layer and independent of the thermal conductivity of the medium or depth of the layer.

Elder, J. W. "Transient Convection in a Porous Medium." Joumaz of Fluid Mechanics 27 (1967): 609-623.

Experimental models were studied using a Hele-Shaw cell and numerical simulation of non-steady free convective flows in a porous medium. Specifically, the flow of a blob of hot fluid released from the base of a porous slab and the development of flow when a portion of the base of a slab is suddenly heated were investigated. The numerical scheme was verified by comparison with visual observations. In addition, the previously reported iterative development of the solutions of the steady-state equations were found to have some similarity when compared with the time dependent numerical scheme. It is concluded that the time required to develop a steady state could be crudely estimated from the number of iterations required.

Faust, Charles R. and Mercer, James W. "An Analysis of Finite-Difference and Finite-Element Techniques for Geothermal Reservoir Simulation." Society of Petroleum Engineers Paper No. 5742. Dallas. (1974).

A two-dimensional (area 1) finite-difference geothermal model is presented, and results are compared with a Galerkin, finite-element model previously developed by the authors. In both models, it is assumed that local thermal equilibrium exists and capillary pressure is negligble. The conservation equations for steam and water are reduced to two nonlinear partial differential equations posed in terms of fluid pressure and enthalpy. These equations are applicable to both hot water (single phase) and vapor dominated (two phase) geothermal reservoirs.

In both the finite-difference and finite-element models the time derivatives are approximated by finite-difference expressions. Several example problems, based on analytical solutions and published experimental data, are used to evaluate the relative merits of the finite-difference and finite-element approaches. Results suggest that the finite-element 
method is more suitable for hot-water reservoirs because of less numerical diffusion and better approximations of boundary and internal geometries (with fewer node points). For vapor dominated systems, the finitedifference model appears superior because it reduces mass and energy balance errors and exhibits less numerical oscillation.

Faust, Charles R. and Mercer, James W. "Mathematical Modeling of Geothermal Systems." Second U.N. Symposium on the Development and Use of Geothermal Resources. San Francisco, 20-29 May 1975: 1635-1641.

The continuity equations for mass, momentum, and energy in porous media are reduced to two nonlinear, partial differential equations in which the dependent variables are fluid pressure and enthalpy. These equations include the effects of phase change and are applicable to both hot-water and vapor-dominated geothermal systems. The equations are solved by a numerical method that combines a Galerkin finite element approximation in space and finite-difference approximation in time. This method yields results which compare favorably with an analytical solution for the one-dimensional steady vertical flow of hot water in a porous medium. Simulation of horizontal flow in a hypothetical reservoir in one and two dimensions indicates the model will adequately treat the boiling phenomenon, the propagation of a two-phase zone around a production site, and subsequent change to a superheated steam state.

Faust, Charles R. Numerical Simulation of Fluid Flow and Energy Transport in Liquid-and Vapor-Dominated Hydrothermal Systems. Ph.D. dissertation, Pennsylvania State University (1976).

A mathematical model for simulating the pressure, temperature and phase-saturation behavior of geothermal reservoirs is rigorously derived on the basis of the balance equations for mass, momentum, and energy in porous media. With appropriate assumptions these equations are reduced to two nonlinear partial-differential equations in which the dependent variables are fluid pressure and enthalpy.

Two alternative numerical models (Galerkin, finite-element and finite-difference) are developed for solving the combined pressure-enthalpy equations. Specifically, these models describe the two-dimensional (areal) unsteady-state flow of mass and energy within a heterogeneous, anisotropic, porous medium containing a single component, single or two-phase fluid (water). Several example problems are presented to illustrate various types of reservoir behavior and to demonstrate the accuracy of the two alternative numerical models.

The results of these examples suggest that the pressure-enthalpy models can be used for geothermal reservoir simulation, that productive geothermal reservoirs require high porosity and permeability, that the Galerkin, finite-element model is better suited for single-phase applications, and that the finite-difference model is better suited for two-phase applications. 
Faust, Charles R. "Summary of Our Research in Geotherma 1 Reservoir Simulation." Workshop on Geothermal Reservoir Engineering. stanford. (Stanford University Press, 1975): 198.

The reservoir modeling effort at the U.S. Geological Survey is reviewed. The initial single-phase finite-element model was successfully used to model the Wairakei field during the initial single-phase period of production. A two-phase model has since been developed by a combined finite-element finite-difference approach. The model has been compared with analytical solutions and experimental results, and is now being applied to an extended simulation of the Wairakei system.

Garg, S. K. and others. Simulation of Fluid-Rock Interactions in a Geothermal Basin. (Systems, Science and Software Report SSS-R74-2431, La Jol1a, 1974).

General balance laws and constitutive relations are developed for convective hydrothermal geothermal reservoirs. A fully interacting rock-fluid system is considered; typical rock-fluid interactions involve momentum and energy transfer and the dependence of rock porosity and permeability upon the fluid and rock stresses. The mathematical model also includes multiphase (water/steam) effects. A simple analytical model based on concentric rock fluid cylinders is employed to study heat transfer into or from a fluid moving in a porous medium. Numerical results show that for fluid velocities typical of geothermal systems (Reynolds number $\ll 10$ ), the fluid and the solid may be assumed to be in local thermal equilibrium. Mathematical averaging techniques of Anderson and Jackson are used to derive a continuum species transport equation for flow in porous media. This method allows the convective and diffusive mechanisms in the continuum representation of species transport to be delineated. An existing computer program is applied to study upwelling of hot water from depth along a fault; the numerical results can be used to explain local temperature inversions occasionally observed in bore hole measurements.

Gou1d, Thomas L. "East Mesa Reservoir." Workshop on Geothemal Reservoir Engineering. Stanford. (Stanford University Press, 1975): 146-152.

Intercomp's reservoir engineering program for the East Mesa reservoir is reviewed. Existing data are being evaluated and correlated with geological information so that an effective well test program can be designed which will provide accurate data for reservoir simulation studies. A combined reservoir and wellbore model is being developed to investigate the effects of various exploitation strategies on the lifetime of the resource, and to investigate aspects of reservoir management such as production well design, spacing and injection well siting and pumping performance. 
Grant, M. A. Asymptotic Solution for the One-Phase Field Model and Lifetime Estimate. (Department of Scientific and Industrial Research, Applied Mathematics Division, Technical Report No. 47, Wellington, New Zealand, 1976).

A single phase geothermal reservoir is modeled by conceptualizing a cylindrical zone of hot water surrounded by cold water. The hot fluid is produced and this generates a drawdown of the surface. Hot fluid flow from the bottom and cold from margins of the hot zone are induced under the pressure gradient. Single-phase one-dimensional conservation equations are written for the hot and cold zones and coupled by maintaining pressure and mass continuity at the hot-cold boundary. Viscosity contrasts between hot and cold water are included and shown to have a significant effect on fluid motion. The model is based on observations at Wairakei, and the resulting predictions closely match the measured performance. A similar approach at Broadlands, however, does not adequately match the observed response.

Grant, M. A. Broadlands - A Gas-Dominated Geothermal Field (Department of Scientific and Industrial Research, Applied Mathematics Division, Technical Report No. 46, Wellington, New Zealand, 1976).

The Broadlands geothermal field in New Zealand is a hot-water system containing a few percent of non-condensable gas (carbon dioxide). This gas has a marked effect on the field response to production. The gas pressures cause boiling at depths of about $2 \mathrm{~km}$, so that bores exploit only the two-phase zone. The initial pressure response to exploitation is dominated by changes in gas pressures, so that conventional resource assessments are misleading. The fact that bores exploit the two-phase region means that they exploit only a confined area near a bore and so are very sensitive to local variations in permeability. This paper presents a model of the response of this field to exploitation and its recovery.

Grant, M. A. Discharge of a Porous Block (Department of Scientific and Industrial Research, Applied Mathematics Division, Technical Report No. 21, Wellington, New Zealand).

Conservation equations are presented and analytically solved for the discharge of a porous block initially containing water at boiling point. The rate of extraction is assumed to be small enough that no spatial variation in properties occurs within one block. The temperature change in the block is plotted against saturation. Diffusivities are calculated as a function of separation and shown to be greater for steam bearing rock than water bearing rock. 
Grant, M. A. Exploitation of a Limited Two-Phase Reservoir (Department of Scientific and Industrial Research, Applied Mathematics Division; Technical Report No. 41, Wellington, New Zealand, 1976).

The pressure, temperature and enthalpy of discharge from a porous block in which boiling occurs are calculated and plotted. The presence of significant gas content is shown to greatly influence the initial pressure response of the field. Comparisons are drawn with the Broadlands geothermal field in New Zealand. The observed large pressure drawdown is attributed to the effects of high gas concentration and the appreciable pressure recovery at shutdown is due to significant recharge into the field.

Gringarten, A. C. "Extraction of Heat from Multiple-Fractured Dry Hot Rock." Geothermics 2 (1973): 119-122.

A brief summary is presented of theoretical solutions for the extraction of heat from multiple-fractured dry hot rock. The analysis is based on an infinite series of parallel uniformly spaced vertical fractures separated by blocks of homogeneous and isotropic impermeable rock. Cold water enters at the bottom of the fractures and the solution is given in terms of dimensionless water temperature at the exit. An example illustrates how a multiple-fractured system provides a more efficient mechanism for heat extraction than a single fracture in dry hot rock.

Gringarten, A. C. and Sauty, J. P. "The Effect of Reinjection on the Temperature of a Geothermal Reservoir Used for Urban Heating." Second U.N. Symposium on the Development and Use of Geothermal Resources (1976): 1370-1374.

A simplified mathematical model is derived for determining the temperature characteristics of an arbitrary number of production wells in a heterogeneous aquifer under arbitrary boundary conditions when the removed water is reinjected at a temperature which is different from the initial temperature. The governing two-dimensional conservation equations are written for a horizonta1, constant thickness and isotropic aquifer with the assumptions that the upper and lower confining beds have infinite thickness and a well defined thermal conductivity in the vertical direction. In addition, the aquifer is assumed to have infinite thermal conductivity in the vertical direction, physical properties independent of temperature and a steady-state flow field. These simplifications allow analytical solutions to be obtained for the time-dependent thermal characteristics of the reservoir for arbitrary production - injection well configurations. 
Gringarten, A. C. and Sauty, J. P. "A Theoretical Study of Heat Extraction from Aquifers with Uniform Regional Flow." Journal of Geophysical Research 80 (1975): 4956-4962.

A mathematical model is presented for investigation of non-steady state temperature behavior of a pumped aquifer during reinjection of a fluid at a temperature different from that of the native water. The two-dimensional heat equation is solved analytically with the assumptions that the flow field is steady, the aquifer is isotropic, convection dominates in the aquifer zone, and fluid properties are independent of temperature. Results are presented in terms of dimensionless parameters for time and temperature and should be helpful in the design of geothermal space-heating projects. Applications to practical cases are also included.

Hardee, Harry C. Heat Transfer in Magma Bodies. (National Technical Information Services Report SAND 76-0011, Springfield, 1976).

Analytical heat transfer techniques are used to relate geological surface evidence and observations to conditions that are likely to exist in magma chambers. The calculations indicate that an upward melting magma system is approximately equidimensional and that injected magmas with very high vertical length/width ratios are likely formed by a forced intrusion process which involves little if any melting or natural convection. Calculations and surface heat flow measurements indicate that the steady-state heat extraction rates for shallow magma chambers with low superheat will probably be of the order of $10 \mathrm{~kW} / \mathrm{m}^{2}$ or less.

Holst, P. H. "Theoretical and Experimental Study of Natural Convection in a Confined Porous Medium." Canadian Journal of Chemical Engineering 50 (1972): 232-241.

Natural convection in a porous medium was modeled by writing force, mass, and energy balances on a differential element of porous medium. The resultant set of partial differential equations, which accounts for temperature-dependent physical properties, was solved numerically after the introduction of a pseudo-stream function. Good agreement was obtained between theoretical and experimental results. Theoretical calculations show that under certain conditions variation of fluid properties with temperature has a significant effect on convective motions. In addition, the orientation of the cell has a significant effect on the flow and temperature patterns.

Holst, P. H. "Transient Three-Dimensional Natural Convection in Confined Porous Media." Intermational Journal of Heat and Mass Transfer 15 (1972): 73-90.

This paper deals with results obtained from the finite difference solution of the equations describing transient natural convection in porous media. A homogeneous, isotropic medium is assumed where fluid 
property variations are considered negligible except where density changes create buoyancy and a combination of constant temperature and insulated boundary conditions are used. The equations are made more amenable to a numerical solution by introducing a vector potential, which may be regarded as the three-dimensional counterpart of the stream function. The equations are initially solved using the linear perturbation approximation, and the critical Rayleigh number is established for the onset of convection.

Numerical solutions of the nonlinear equations indicate that under certain conditions three-dimensional motion. would result in significantly higher heat transfer rates across the porous medium than two-dimensional motion at the same Rayleigh number. The convection pattern of the three-dimensional motion is illustrated by isometric projections of isothermal surfaces and two-dimensional projections of streaklines which trace the path of a fluid particle.

Horne, Roland N. and O'Sullivan, Michael J. "Numerical Modeling of the Wairakei Geothermal Reservoir." Society of Petroleum Engineers Paper No. 6536. Dallas. (1977).

A hypothetical geothermal aquifer that depletes the fluid saturating its upper regions during production is considered. This production process is modeled after the Wairakei geothermal aquifer, where water table and consequent pressure drawdown have been observed in the regions of maximum fluid extraction. The conservation equations are presented in twodimensional axisymmetric form for a single-phase reservoir with a free boiling surface moving at a rate determined by the rates of production and recharge (both natural and by injection). The equations are solved numerically using a finite difference technique. Two exploitation strategies were considered. Dwindling production over a long period of time where recharge is from natural seepage from the surface and sustained production over a shorter period where cold water injection is used to maintain reservoir pressures were found to be fairly similar in terms of the total energy recovered. However, dwindling production does have the advantage that fluid withdrawal from deeper levels is stimulated. The model can be used in conjunction with physical parameters of a particular aquifer to predict the time taken to reach pressure equilibrium and the output at that time for different production strategies.

Horne, R. N. "Oscillatory Convection in a Porous Medium Heated from Below." Journal of Fluid Mechanics 66 (Part 2, 1974): 339-352.

The stability of natural convective flow in a porous medium heated both uniformly and non-uniformly from below is studied in order to determine the possibility of oscillatory and other unsteady flows, and to explore the conditions under which they may occur. The resuits of the numerical work are directly comparable with experiments using a Hele-Shaw cell and also, in the uniformly-heated case, with the results of other. investigations. It is shown that for the uniformly heated case there exist two distinct possible modes of flow, one of which is fluctuating, 
the other being steady. However, in the non-uniformly heated case, the boundary conditions force the solution into a unique mode of flow which is regularly oscillatory when there is considerable non-uniformity in the heat input at the lower boundary, provided that the Rayleigh number is sufficiently high.

Horton, C. W. and Rogers, F. T., Jr. "Convection Currents in a Porous Medium." Journal of Applied Physics 16 (1945): 367-370.

Convection of fluid through a permeable medium as a result of a vertical temperature gradient where the aquifer is bounded above and below by a perfectly conducting medium is considered. The conservation equations are solved for the onset of convection by approximating the temperature field with a small linear deviation from equilibrium. The minimum temperature gradient required for the onset of convection is compared with the value for convection in a viscous fluid. Application of the theory to a practical case does not appear to be consistent with observed phenomenon.

Hunsbedt, Anstein and others. "Energy Extraction Experiments in the SGP Reservoir Model." Third Stanford Geothermal Reservoir Engineering Workshop. Stanford. (Stanford University Press, 1977): 299-301.

Heat and fluid extraction experiments were conducted in a laboratory model containing rock systems with permeabilities of approximately 1 darcy and porosities of $21 \%$. The nonisothermal fluid-production energy-extraction process defined as in-place boiling, where fluid is drawn from the top of the reservoir, was compared with previously reported similar experiments (which also included sweep and steam drive recovery strategies) in fractured rock of high porosity and permeability. A significant improvement in energy extraction efficiency was noted, and it was concluded that natural convection and heat transfer in the rock is not impeded by the lower permeability formation. Further investigations will reveal to what point the permeability can be lowered without significantly deteriorating the energy extraction effectiveness of the in-place boiling process.

Hunsbedt, Anstein. "Energy Extraction Experiments in the SGP Reservoir Model." Geothermal Resources Council Iransactions 2 (Part 1, 1978): 299-301.

Experiments were conducted in a laboratory geothermal reservoir model utilizing rock systems with several characteristics resembling high permeability, fracture-stimulated systems. Three non-isothermal energy extraction and production processes, referred to as in-place boiling, sweep, and steam-drive, were considered during the early phases of the study. The results indicate that the sweep process, where cold water is injected into the formation, is the most efficient extraction process. The in-place boiling process (pressure reduction induces 
boiling in the formation) is slightly less efficient with the steam drive process being considerably inferior to the others. Future work will concentrate on modeling of systems with representative porosities and permeabilities.

Hunsbedt, Anstein. Laboratory Studies of Stimulated Geothermal Reservoirs. Ph.D. dissertation, Stanford University (1976).

Improved recovery of geothermal energy from artificially stimulated systems by in-place flashing was studied experimentally. The laboratory model used produced steam at initial pressures and temperatures up to $800 \mathrm{psig}$ and $500^{\circ} \mathrm{F}$, either with or without simulated geofluid recharge. Analytic models based on mass and energy balances assuming uniform axial temperature distribution were developed to describe the experimental pressure and temperature transients. Results show that thermal energy stored in fractured rock can be extracted effectively by reducing system pressure to allow in-place flashing. Recovery of energy from the laboratory model systems studied ranged from 1.25 to 2.57 times the energy extractable by flashing the fluid alone. The recovery factors depend on the initial pressure, the rock porosity, the rock energy extraction fraction, the external heat transfer parameters, and the enthalpy of the recharge fluid. Although improved geothermal energy recovery from stimulated reservoirs by in-place flashing appeared promising, the feasibility of this extraction scheme is yet to be demonstrated in field applications.

Hunsbedt, A., Kruger, P. and London, A. L. Laboratory Studies on NonIsothermal Fluid Production from Fractured Geothermal Reservoirs. (American Society of Mechanical Engineers, New York, 1977). Nonisothermal processes are identified as the means of enhanced energy extraction from geothermal systems. Two methods of enhanced energy extraction are proposed-artificial circulation of water and a "steam drive" process where production occurs from a location below the steam/liquid interface. The feasibility of these two processes was studied in a $17 \mathrm{ft}^{3}$, high permeability, laboratory model of artifically fractured rock. The system was capable of operating at a maximum pressure of 800 psig at $500^{\circ} \mathrm{F}$ temperature.

It is concluded that by recharging cold water at one location while producing hot water at a different location at pressures higher than saturation, the energy stored in the fractured rock media could be successfully extracted. The steam drive process, however, proved ineffective because boiling of the natural or recharged water present in the reservoir tended to be suppressed by noncondensable gases in the steam cap. The experimental results were used to build an analytical model which was successfully applied to large-scale hypothetical systems. 
Hunsbedt, Anstein. "Recovery of Energy from Fractured Geothermal Reservoirs." Society of Petroleum Engineers Paper No. 5875. Dallas (1976).

This paper presents investigations designed to explore (1) conditions for optimum energy extraction, (2) rock heat transfer characteristics, (3) moving flash fronts, (4) fluid withdrawal reservoir pressure behavior, (5) effects of cool and hot fluid recharge, and (6) cyclic production/recharge operation of fracture stimulated geothermal systems. The experiments were carried out in a large-scale laboratory model simulating the highly fractured region of an originally low permeability hydrothermal reservoir. The paper clearly shows that the thermal energy. stored in fractured rock can be extracted effectively by lowering the system pressure by steam production such that boiling is initiated within the rock matrix. The experimental rock energy extraction fractions ranged between 0.75 and 1.18 with an estimated uncertainty interval of $\pm 3.5 \%$.

Hunsbedt, A., Kruger, P. and London, A. L. "Recovery of Energy from Fracture-Stimulated Geothermal Reservoirs." Joumal of Petroleum Technology 29 (1977): 940-946.

Geothermal energy recovery by in-place boiling was studied in a laboratory simulated model of a fracture-stimulated geothermal reservoir heated to $500^{\circ} \mathrm{F}$ and at a pressure of $800 \mathrm{psig.} \mathrm{Nonisothermal} \mathrm{heat-}$ transfer processes in fractured rock systems, as opposed to porous media, are the principle interest of the paper. The study was designed to explore (1) conditions for optimum energy extraction, (2) rock heattransfer characteristics, (3) moving flash fronts, (4) fluid-withdrawal reservoir pressure behavior, (5) effects of cool and hot fluid recharge, and (6) cyclic production recharge operation.

An analytical model to evaluate rock energy extraction was developed, and agreement between predicted and experimental results was satisfactory.

Isita, J. "Low Permeability of a Thick Geothermal Aquifer in a Highly Fractured Area as Necessary Requisite for High Steam Production." U.N. Symposium on the Development and Utilization of Geothermal Resources. Pisa, 22 September-1 October 1970: 684-697.

The factors affecting storage of heat in a geothermal aquifer are discussed. The combinations of high reservoir transmissability, low. natural groundwater flow, and a high density of fractures are identified as being important for satisfactory reservoir performance. Well drawdown interference, and long-term performance are discussed in terms of reservoir characteristics. Examples of well reservoir interaction in the Cerro Prieto geothermal field are given. 
Isokrari, Ombo F. "Natural Gas Production from Geothermal Geopressured Aquifers." Society of Petroleum Engineers Paper No. 6037. Dallas. (1976)

A two-phase two-dimensional numerical simulator is used to model the production performance of isothermal and non-isothermal deformable reservoirs. The dynamic response of the rock is treated by approximate constitutive relationships for porosity as a function of pressure and temperature. Three types of reservoir are considered: (1) reservoir water is undersaturated with natural gas, (2) reservoir water is fully saturated, and (3) free gas exists with the water being nearly immobile in the reservoir.

Finite difference techniques are employed to solve the equations and either areal or cross-sectional studies can be performed. Simulations were performed with and without influx and the simulated production history plotted in terms of a bottom hole pressure to compressibility ratio. It is concluded that large volumes of natural gas can be produced from geopressurized reservoirs. Reservoir $11 \mathrm{fe}$ is prolonged if gas is produced from the water-saturated area in reservoirs having gas caps. Cumulative production and pressure decline curves are difficult to interpret and fluid expansion, formation compressibility, and compaction are the main production driving forces.

Isokrari, Ombo F. Nhonerical Simulation of United States Gulf Coast Geothermal Geopressured Reservoirs. Ph.D. dissertation, University of Texas (1976).

Multiphase fluid flow equations and constitutive relationships are derived to describe deformable, anisotropic, heterogeneous and nonisothermal reservoirs such as the Gulf Coast geopressured geothermal aquifers. Reservoir drive mechanisms considered include fluid and rock matrix compressibilities, formation compaction, shale-water influx, fluid expansion, and natural gas in solution. Gravity, capillarity, and pressure and temperature dependence of fluid densities, viscosities, natural gas solubility in water, and gas deviation factors are also considered. The reliability of the model was tested by simulating the production history of the Anderson " $L$ " reservoir. Good agreement was obtained between observed and simulated performance.

James, Russe11. "Wairakei and Larderello: Geothermal Power Systems Compared." New Zealand Joumal of Science 11 (1968): 708-719.

A comparative study is made of the exploitation for electric power of the superheated steam reservoir at Larderello, Italy, and the pressurized hot-water aquifer at Wairakel, New Zealand. It is concluded that these apparently dissimilar fields are both based on a liquid phase, the only significant difference being the greater relative depth of the underlying water at Larderel10. It is submitted that dry-steam reservoirs can only exist with their top levels comparatively close to the ground surface and at a pressure not exceeding about 30 atmospheres. This is due to the nature of the steam-water-saturation curve in this 
region. Production trends for both systems are predicted. The influence of dissolved chemicals in the water phase and noncondensable gases in the steam phase is also considered.

Kasameyer, P. W. and Schroeder, R. "ThermaI Depletion of Liquid-Dominated Geothermal Reservoirs with Fracture and Pore Permeability." Workshop on Geothexmal Reservoir Engineering (1975): 249-257.

A geothermal reservoir is modeled as a combination of rock, fluid in interstitial pore space, and fluid in fractures. Three coupled linear rate equations are solved for the time dependence of the average temperature of the rock, pore fluid and fracture fluid. By averaging temperature over the entire reservoir and assuming all the fluid is reinjected, effects such as drawdown are ignored, and an upper bound for the reservoir heat content and average wellbore temperature is determined as a function of time. Heat transfer from the rock to the fluid by conduction, both in fractures and in pores and by the flow of fluid through the pores, is considered. The lifetime of a geothermal power plant is found to be strongly dependent on the fracture spacing and fracture permeability of the geothermal reservoir.

Kassoy, D. R. "Heat and Mass Transfer in Models of Undeveloped. Geothermal Fields." Second U.N. Symposium on the Development and Use of Geothermal Resources. San Francisco, 20-29 May 1975: 1707-1711.

A brief summary is given of the nondimensional equations and relevant parameters to be used for describing convection processes in the geothermal setting. Characteristic estimates of flow rates and pressure variation (beyond the hydrostatic value) are given. It is shown that the complete equation system can be reduced in complexity by neglecting terms which describe physically insignificant effects. The resulting equations are used to consider the onset of convection in a horizontal saturated porous slab when a realistic model of viscosity variation is used. It is shown that the classical constant viscosity prediction is in error both quantitatively and qualitatively. A calculation is made for the rate of convective upflow in a model fault zone. It is shown that under appropriate conditions it is possible to transport as much as 2 to $3 \times 10^{6} \mathrm{~kg} /$ day up a fault system with a horizontal crosssectional area of only $0.3 \mathrm{~km}^{2}$.

Kassoy, D. R. "Summary Description of Research Activities." Workshop on Geothexmal Reservoir Engineering. Stanford. (Stanford University Press, 1975): 23-25.

The geothermal reservoir engineering research program at the University of Colorado is described. Physical characterization studies of the Mesa anomaly in Imperial Valley, models of cyclic stress variations induced by tidal forces, pore collapse due to reduction in pore pressure, and heat and mass transfer studies in reservoirs exhibiting some faultcontrolled features are mentioned. 
Kassoy, D. R. Transport of Mass and Energy in Porous Media Due to Natural Convection: The Geothermal Basin Problem (National

Technical Information Service Report PB-212917., Springfield, 1976).

A research program is outlined which deals with the modeling of physical processes occurring in liquid-dominated geothermal systems. General heat and mass transport equations are derived along with a model describing deformation due to periodic earth tide loading and constitutive relations for the deformation and failure of rocks in saturated, high temperature and pressure environments. An analys is of the onset of convection and resulting motions and heat transfer in fault-dominated geothermal systems is presented.

Field data from the East Mesa anomaly in California are used to characterize the physical and structural nature of the system. It is submitted that the East Mesa system is a fault-dominated single-phase convection system and is treated accordingly. Temperature and flow fields are described for the development of steady state conditions in a two-dimensional numerical simulation of the system. It is concluded that flow and temperature configurations in faulted systems depend upon fault-zone geometry, Rayleigh number, and horizontal aquifer characteristics.

Kassoy, D. R. and Zebib, A. "Variable Viscosity Effects on the Onset of Convection in Porous Media." Physice of Fluide 18 (1975): 1649-51.

The two-dimensional conservation equations are solved for the onset of convection in a porous slab using a linearized stability analysis technique. The critical Rayleigh number for the onset of convection is dramatically reduced from the constant viscosity value of $4 \pi^{2}$ when a realistic viscosity-temperature relationship is included. The result is that the temperature differences required for instability in a given slab are less than that predicted by constant property theory; or, for a given temperature difference, the critical permeability and/or the slab height is reduced. The streaml ine and isotherm patterns are also distorted in a vertical direction.

Kent, Thomas L. and Pierson, Roy G. "Three Dimensional Geothermal Reservoir Simulation." Society of Petrolewon Engineers Paper No. 6104. Dazlas. (1978)

A three-dimensional, finite-difference model for the simulation of geothermal reservoirs is described. The model is designed to simulate geothermal reservoirs that contain water in any of its vapor or liquid states and provisions are included for properly treating changes of state during a time step.

Mass and energy balances are solved simultaneously using an implicit pressure/explicit saturation (IMPES) formulation. An implict treatment of production rates, capillary pressure, and transmissibilities is included as an option. Thus, entire field, cross-sectional, or individual well studies can be performed. 
The model was applied to four specific problems in one, two and three dimensions. The one-dimensional solution compared favorably with experimental results obtained from flow in a core initially filled with subcooled liquid. The two- and three-dimensional examples traced the production performance of a reservoir from subcooled liquid through a two-phase condition to superheated steam.

Knapp, R. M. and others. "An Analysis of Production from Geopressured Geothermal Aquifers." Society of Petroleum Engineers Paper No. 6825. Dallas. (1977)

A comprehensive theoretical formulation is presented for the important thermomechanical processes operative in a geopressured geothermal reservoir. The formulation includes the effects of four major drive mechanisms (pore fluid compressibility, reservoir rock compaction, the evolution of dissolved natural gas, and the influx of water from adjacent shale formations) expected to be operative during the productive life of the reservoir. Finite difference techniques were used to solve the governing equations describing mass conservation, momentum and energy transport for two flowing phases in a multidimensional heterogeneous reservoir. Constitutive equations were used to describe the changes of fluid properties and reservoir parameters with changes in reservoir pressure and temperature. A series of test calculations were performed to assess the sensitivity of reservoir performance to the gas in solution, sediment compaction, and the reinjection of waste fluids. It is concluded that sediment compaction and water from interbedded shales can be significant depletion drive mechanisms in geopressured aquifers. The natural gas drive will probably not exceed the water expansion unless there is a significant initial gas saturation. Reinjection of produced fluids into geopressured geothermal aquifers will be desirable to increase the recovery of both thermal energy and natural gas.

Knapp, Roy M. "Aspects of Numerical Simulation of Future Performance of Geopressured Geothermal Reservoirs." Second Geopressured Geothermal Energy Conference. University of Texas, Austin, 23-25 February 1976: 102-161.

Two-dimensional areal and cross-sectional numerical models are developed to simulate the performance of geothermal geopressured reservoirs. The effects of anisotropy, water influx, and compaction as a result of production are included. Constitutive equations relating pore pressure and rock compaction, and equations of state for the gas-water mixture are presented. The coupled conservation-constitutive equation set is solved numerically using finite difference techniques to describe the behavior of water and gas phases in the reservoir fluids and the behavior of the rock matrix. The results indicate that geopressured reservoirs of the type located along the Gulf Coast of the United States are capable of sustaining significant fluid production over a number of: years. 
Kononenko, G. N. "Coolant Filtration in Man-made Underground Circulation Systems." Journal of Fluid Mechanics 3 (1974): 95-98.

Based on potential theory, an electric analog of filtration flow in porous media was performed. Recommendations are given on determining the pressures in boreholes as a function of their number for a given volume of pumped coolant, and the pressure field is presented for a system consisting of two cavities.

Kruger, Paul. "Physical Models of Stimulated Geothermal Reservoirs." Workshop on Geothermal Reservoir Engineering. Stanford. (Stanford University Press, 1975): 169-173.

Physical models have been developed at Stanford University to evaluate optimum performance of fracture-stimulated geothermal reservoirs. Three such efforts reported in this summary are: laboratory simulation of an explosion-produced rubble chimney to obtain experimental data on the extractability of heat from hot rock by in-place boiling; heat and mass transfer transients with individual porous rock fragments to compare their relative importance in stimulated systems; and measurement of radon emanation from geothermal reservoirs as a tracer for reservoir engineering studies.

Kruger, Paul. "Stimulation and Reservoir Engineering of Geothermal Resources." Stanford Geothermal Program, TR-1. (Stanford University Press, 1974).

The geothermal reservoir engineering research program at Stanford University is reviewed. The laboratory experimental program is discussed, including investigation of rock-water interactions and energy extraction from fracture systems, thermal stress fracture initiation and capillary pressure and absorption effects caused by the nonisothermal flow of bolling fluids. The development of methods for using radon as an internal tracer for examination of reservoir properties is outlined and recent developments in well test analysis for two-phase reservoirs are presented. Details of progress in the above research areas have been made available to the geothermal community through meetings and written publications. In addition, the form and purpose of the weekly geothermal seminars and the annual reservoir engineering workshop held at Stanford are outlined. The program objectives for the next year are established and their relevance to the timely development of geothermal energy is discussed.

Kuo, Ming-Ching T, Kruger, Paul, and Brigham, William E. "Heat and Mass Transfer in Porous Rock Fragments." Stanford Geothermal Program, TR-10. (Stanford University Press, 1975).

To evaluate the important mechanisms related to heat extraction from highly fractured or porous media, separate experiments were designed and conducted to determine the mass transfer and the heat transfer rates 
from well defined artificial porous rocks to surrounding fluid under controlled transient conditions. The rate of mass transfer between tritiated-water saturated rocks to surrounding fluid was determined by radioactivity measurement. The rate of heat transfer was determined by quartz thermometry. The measured thermal diffusivity of the rock fragments was of the order of $10^{-2} \mathrm{~cm}^{2} / \mathrm{sec}$ and the measured effective mass diffusivity of the micropore water was of the order of $10^{-6} \mathrm{~cm}^{2} / \mathrm{sec}$. The data were used to analyze energy transfer for a model involving both intrasphere and interface transport steps.

Lapwood, E. R. "Convection of a Fluid in a Porous Medium." Proceedings of the Cambridge Philosophical Society 44 (1948): 508-521.

The onset of fluid convection in a two-dimensional porous layer heated from below was studied. With the assumption that Darcy's law applies and using the Boussinesq approximation, the equations for heat and mass transfer were formulated. These were solved using linear perturbation approximations. Marginal stability and convective motion were studied for a number of boundary conditions. It was found that marginal stability was characterized by a Rayleigh number of $4 \pi^{2}$. Finally, heat flow in the presence of convection is analyzed and the influence on borehole temperature is discussed.

Lasseter, T. J. "Multiphase Multidimensional Simulation of Geothermal Reservoirs." Second U.N. Symposizon on the Development and Use of Geothermal Resources. San Francisco, 20-29 May 1975: 1715-1723.

A mathematical method of modeling geothermal reservoirs has been developed using a computer program called SHAFT (Simultaneous Heat and Fluid Transport). This program solves numerically, using an integrated finite difference technique, the coupled equations describing the simultaneous transport of mass and energy by a one- or two-phase fluid in porous media for transient or steady-state systems in one, two, or three dimensions. The governing equations are set up in terms of two expressions, one for flow and one for internal energy. Solutions are obtained by solving for two unknowns, density and internal energy, as a function of time and position within the system. Details of the deve1opment of flow and energy equations are presented. Two examples are given of the application of SHAFT to a liquid and vapor dominated geothermal reservoir which both operate in the two-phase region.

Lasseter, T. J. The Nrmerical Simulation of Heat and Mass Transfer in Multi-Dimensional Two-Phase Geothermal Reservoirs. (75-WA/HT-71) (American Society of Mechanical Engineers, New York, 1975).

This paper describes the development of a computer program to solve numerically, using an integrated finite difference technique, the coupled equations describing the simultaneous transport of mass and energy by a one- or two-phase fluid in porous media for transient 
or steady-state systems in one, two, or three dimensions. The equations are solved for the two dependent parameters, density and internal energy, by alternatively solving the decoupled equations for each variable at each time step. The program will allow different fluids as long as they are confined to separate regions in the system. In general, the fluids must be pure or of uniform composition in both phases. For systems where the flow field is essentially independent of the temperature distribution, the flow field is only computed once at the beginning of the problem and subsequently only energy calculations are made. A two-dimensional mesh generator and graphics capabilities are also included. The accuracy of the program is shown by comparison with analytic and other numerical solutions for single-phase convective heat transfer in porous media systems. Detailed results for three different types of hypothetical geothermal systems are presented: a liquid-dominated, a vapor-dominated, and a liquid-vapor system.

Leverett, M. C. Dimensional Model studies of Oil Field Behavior. (AIME Technical Publication No.' 1413, New York, 1942).

Two dimensionally scaled models for idealized oil fields are presented. One simulates the well and sand under radial flow and the other simulates linear flow through layered sand. Construction and operation of the models are described and typical data are presented. Permeability-viscosity ratio is treated as a single variable. The models are designed to study the desirability of various methods of well completion and the effects of various factors of oil recovery from layered sands. The superiority of these models over unscaled models is pointed out.

Lowell, R. P. "Circulation in Fractures, Hot Springs, and Convective Heat Transport on Mid-Ocean Ridge Crests." Geophysical Joumal of the Royal Astronomical Society 40 (1975): 351-365.

Thermal springs are discussed on the basis of fluid flow in fractures. A steady-state model of one-dimensional free convective vertical flow in fractures shows that a fracture a few millimeters wide can carry a substantial convective flow. The steady-state model was also used to estimate conductive temperature losses and the depths of circulation for thermal springs in southeastern United States. Timedependent models were also developed to show the effect of circulation on the regional geothermal gradient. Both hydrostatically driven and free convective flows are discussed. Hydrothermal circulation in midocean ridges is interpreted using the time-dependent model.

Marsha11, D. C. "Development of a Theory of the Wairakei Geothermal Field by the 'Simplest Cases First' Technique." U.N. Symposizm on the Development and Utilization of Geothermal Resources. Pisa, Italy, 22 September - 1 October 1970: 669-676.

A theory is required to relate the pressure at depth in the Wairakei geothermal field to the history of hot water discharge. This 
will enable prediction and control of the performance of the field. The aim is to discover the simplest theory which relates pressure to discharge, in the hope that some of the phenomena which are undoubtedly occurring may nevertheless be having a neglible effect.

A simple model is assumed, incorporating a rising, vertical, axisymmetrical column of hot water which remains as pure liquid phase right to the ground surface. This model gives results consistent with the hypothesis that convective buoyancy forces cause the upward flow of hot water. The results also show that drawdown of the liquid surface would occur, which again fits the observed performance.

Marshal1, D. C. "Preliminary Theory of the Wairakei Geothermal Field." New Zealand Joumal of Science 9 (1966): 651-673.

Hydrostatic theories cannot account for the pressure changes which have occurred in the Wairakei geothermal field. The pressures at three representative dates can, however, be predicted on the theory that the hot water flows according to Darcy's law under the influence of buoyancy forces resulting from the surrounding cold groundwater, allowance being made for the effect of the uncased parts of the bores in increasing the vertical permeability of the hot region. A steady rate of drawoff from the bores will result after a period on the order of one year, giving an almost steady pattern of pressure with depth, lower pressures corresponding to greater drawoff. The ultimate source of most of the water withdrawn is the cold groundwater surrounding the hot region, which is heated by the hot rock through which it flows and in so doing, cools the outer parts of the hot region. The contraction of the hot region will cause a slow fall in pressure if the rate of drawoff is held constant.

Martin, J. C. "Analysis of Internal Steam Drive in Geothermal Reservoirs." Joumal of Petroleum Technology 27 (1975): 1493-1499.

Petroleum reservoir analysis methods are applied to simple closed geothermal reservoirs that produce by internal steam drive. The fundamental assumption is that fluid flow in a geothermal reservoir can be treated as flow through a porous medium, and Darcy's law and relative permeabilities are applicable. The relationship between pressure, temperature, and production as a function of fluid mobility is derived. Pressure and temperature relationships versus cumulative production and saturation are calculated for a range of initial reservoir conditions from single-phase steam to pressurized water. The hot water reservoirs show complicated characteristics changing from hot water to steam as they are produced. Gravity segregation of the steam and water phases in the upper portion of the reservoir indicates that wells should be completed at fairly shallow horizons to maximize the energy recovery. 
Martin, J. C. "Fluid Flow in Geothermal Reservoirs." Workshop on Geothermal Reservoir Engineering. Stanford. (Stanford University Press, 1975): 42-44.

The pressure-temperature behavior of geothermal reservoirs produced from various initial thermodynamic conditions is discussed. Internal steam drive in hot water systems and the essentially isothermal production from steam reservoirs is qualitatively described.

Marconcini, R. and others. "Modeling Vapor-Dominated Geothermal Reservolrs." Proceedings of the Larderello Workshop on Geothermal Resource Assessment and Reservoir Engineering. Pisa, Italy, 1977: 256-298.

The development of a numerical model of the Serrazzano geothermal field in Italy is described. The model uses an integrated finite difference method to solve the three-dimensional conservation equations. The simulator grids are constructed to approximate the geology of the field, and boundary conditions are based on observed structural features. Production data are used to define the physical parameters and initial conditions of the reservoir. The results of the simulation are compared with avallable production history to verify the applicability of the simulator. The model is reputed to be the first attempt to numerically model the performance of a vapor-dominated reservoir. There is much speculation over the role of water in vapor-dominated systems, and it is felt that the model will help clarify the physical processes taking place in such systems.

McNabb, A. Geothermal Physics. (Department of Scientific and Industrial Research Technical Report No. 32, Wellington, New Zealand, 1975).

Various models are postulated concerning the size and dynamics of the geothermal heat source in the Taupo Volcanic Zone. The transport of heat and mass (water, gases, and chemical species) is discussed in terms of free convection and thermal diffusion of intrusive magmas. Various results from surface geological, chemical, and hydrological measurements are used in support of these models. Transport of heat from the deep hot pluton to the shallow groundwater aquifer and the subsequent aquifer temperature is postulated on the basis of a layer of concentrated salt solution. In addition, the depth to the heat source is estimated on the basis of the heat flux and thermal gradients in the aquifer.

The groundwater hydrology is also discussed and the contribution of a hydrostatic driving force in addition to buoyancy-generated convection is outlined. It is concluded that the convective field is considerably complicated by the chemical deposition and thermal stress fracturing. 
Meng, C. Y. and Shaw, P. T. "Study of Heat Conduction Models of Geothermal Energy Reservoirs." U.N. Symposizm on the Deveiopment and Vtilization of Geothermal Resources. Pisa, Italy, 22 September - 1 October 1970: 1270-1274.

Temperature anomalies in a rock formation are considered as direct evidence of the existence of geothermal reservoirs. Thermal conduction models of different presumed geothermal reservoir shapes are discussed. A geothermal reservoir is considered to be a constant hot body overlain by uniform rock formations. The temperature distribution and conduction heat flow in the overlying formation are expressed theoretically with the Laplace equation.

Temperature distribution in the overlying rock formation may be represented by the surfaces of constant temperature whose shapes are closely related to the shape of the hot body. The shape of temperature curves at equal depth in a vertical cross section also reflects the depth, shape, and size of the geothermal energy reservoir. Groundwater flow will disturb the shape of the isothermal surfaces away from the purely conductive solution.

Mercer, James W. Jr. Finite-EZement Approach to the ModeZing of Hydrothermal Systems. Ph.D. dissertation, University of Illinois (1973).

The equations of mass and energy transport are derived for singlephase flow in porous media. A numerical scheme is developed to solve the equations for a two-dimensional areal model of a hydrothermal system which incorporates a Galerkin finite-element approximation for spatial derivatives and finite difference approximation for temporal derivatives. The assumptions used in deriving the model are discussed in detail. The model is used to simulate both the steady-state and nonsteady-state production performance at the Wairakei field in New Zealand. The steadystate simulation reveals that an overlying aquitard is an important prerequisite for the formation of hydrothermal fields. Reservoir performance for the first ten years of production at Wairakei was well correlated with the nonsteady-state model.

Mercer, James W. Jr., Pinder, George, and Donaldson, Ian G. "A Galerkin Finite Element Analysis of the Hydrothermal System at Wairakei, New Zealand." Journal of Geophysical Research 80 (1975): 26082621.

A two-dimensional areal model was developed in which the heat and mass transport equations were solved using finite difference approximations for the time derivatives and Galerkin finite element approximations for the space derivatives. A single-phase simulation was carried out of a structurally simplified representation of the hot-water hydrothermal field at Wairakei, New Zealand. The two-dimensional areal analysis was performed for steady state and transient flow conditions, allowing vertical flow of heat and fluid through an overlying aquitard. Calculated temperature and pressure patterns correlate well with observed patterns 
for the first ten years of production when increasing quantities of steam in the production aquifer invalidated the assumption of singlephase flow.

Mercer, James W. Jr. "Galerkin Finite Element Simulation of a Geothermal Reservoir." Geothermice 2 (Numbers 3-4, 1973): 81-89.

The equations describing fluid flow and energy transport in a porous medium were used to formulate a model capable of simulating the transient response of a hot-water geothermal reservoir. The resuiting equations were solved using a numerical scheme which combined the finite element approach with the Galerkin method of approximation. Application of this numerical model to the Wairakei geothermal field demonstrates that hot-water geothermal fields can be simulated using numerical techniques currently available and under development.

Mercer, James W. Jr., Faust, Charles R., and Pinder, George F. "Geothermal Reservoir Simulation." Conference on Resecrch for the Development of Geothermal Energy Resources, 23-25 September 1974: 256-267.

The governing equations for energy and mass transport in porous media are presented. The assumptions used to obtain the equations are outlined and include: Darcy's law is applicable, capillary pressure between the steam and water is negligible, compression work is negligible, the properties of steam and water are described by the saturation curve in the two-phase zone, porosity is a function of temperature, and thermal equilibrium exists locally between the rock and fluid allowing a generalized thermal dispersion tensor to be defined. The equations are written in terms of the two dependent variables, pressure and enthalpy. Techniques for solving the resulting coupled nonlinear partial differential equations are briefly discussed.

Mercer, James W. Jr. and Faust, Charles R. "Simulation of Water- and Vapor-Dominated Hydrotherma 1 Reservoirs." Society of Petroleum Engineers Paper No. 5520. Dalzas. (1975).

This paper presents a two-dimensional (areal) two-phase (steamwater) model for the numerical simulation of both water- and vapordominated hydrothermal reservoirs. The model describes transport of heat and flow of compressed water, steam-water mixtures, and superheated steam in a porous medium. The conservation equations for steam and for water are combined thereby eliminating mass transfer terms and, assuming that capillary pressure is negligible and that conditions of local thermal equilibrium prevail, are reduced to two nonlinear partial differential equations in which the dependent variables are fluid pressure and enthalpy.

The two partial differential equations are solved simultaneously using a Galerkin finite element technique for the spatial terms and finite difference techniques for the temporal terms. The model is 
applied to example problems consisting of a hypothetical hot-water reservoir and a hypothetical two-phase reservoir. Model calculations are compared with calculations from other numerical work involving a finite-element model that is restricted to the flow of compressed water, and a finite-difference model that is restricted to the two-phase region.

Miller, F. G. and others. "Reservoir Engineering Aspects of Fluid Recharge and Heat Transfer in Geothermal Reservoirs." Geothermal Resources Council Transactions 2 (Part 2, 1978): 449-452.

Natural recharge of fluid-filled geothermal reservoirs is shown to be important in the developing of these reservoirs and in increasing geothermal energy reserves. Literature is discussed dealing with water influx in oil and gas reservoirs which rely on pressure-time history. The heat transfer processes important to geothermal reservoir production are discussed in terms of an analogy with the methods developed for water and steam injection stimulation processes in the oil industry. A problem with the application of these techniques to geothermal systems concerns the definition of the reservoir boundary. It appears the definition of "cool" water must be based on the lower temperature limit at which water can be economically used. An example illustrates the ease with which the established oil industry techniques can be adapted to the problem of cool water encroachment in geothermal reservoirs.

Moench, Allen F. Simulation of Steam Transport in Vapor-Dominated Geothermal Reservoirs (U.S. Geological Survey Open File Report 76-607, Men1o Park, California, 1976).

A vapor-dominated geothermal reservoir is conceptualized as a one-dimensional column of porous or highly fractured rock initially filled with a mixture of saturated steam and liquid water under high pressure. A simple finite-difference technique is used to solve the flow and energy equations simultaneously for temperature and pressure. Analysis involves evaluation of pressure, temperature, and liquid-water saturation distributions when steam is produced at either constant discharge or constant pressure.

Comparisons of computed saturation profiles with previously published results lead to the conclusion that movement in the liquid phase can be neglected at low moisture content. Temperature and pressure changes when steam is produced at constant or variable discharge, temperature- and discharge-versus-time variations when steam is produced at constant pressure, and the existence of superheated steam is investigated. Results also demonstrate that temperature changes due to fluid expansion or compression may be significant when dealing with superheated steam. 
Morrison, H. L. "Convection Currents in Porous Media. II. Observation of Conditions at Onset of Convection." Journal of Applied Physics 20 (1949): 1027-1029.

Small-scale experiments were carried out for determining the minimum vertical thermal gradient which is required to cause convection in liquids entrapped in porous media. Observations relative to the onset of convective flow in unconsolidated sands indicate that theories which assume constant fluid viscosity and thermal expansibility predict minimum gradients which are excessive by considerable amounts. The ratio of theoretical to observed gradients is derived on the basis of fluid and porous media properties.

Mundry, E. "Mathematical Estimation Concerning the Cooling of a Magmatic Intrusion." U.N. Symposizm on the Development and Utilization of Geothermal Resources. Pisa, Italy, 22 September 1 October 1970: 662-668.

The cooling of intruded magmatic bodies has been calculated for a number of simple models according to the heat conduction theory. The effect of latent heat being released during the solidification of the magma can be determined by means of numerical models. Assuming specific values for the latent heat, the temperature distribution is graphically presented as a function of time for spherical, cylindrical and plateshaped models. Calculations of this kind have been carried out for specific basaltic intrusions in the western part of Germany in order to explain the coalification observed.

Nathenson, Manuel. "Reservoir Factors Determining the Fraction of Stored Energy Recoverable from Hydrothermal Convection Systems."

Workehop on Geothermal Reservoir Engineering. Stanford. (Stanford University Press, 1975): 50-51.

Two possible methods for extracting energy from a liquid-filled volume of porous and permeable rocks are analyzed. First, a porous, permeable volume virtually closed to inflow of water is produced by bolling to steam using the energy in the rock. The second method assumes that natural and artificial recharge of cold water is used to recover much of the heat from the reservoir by means of a sweep process.

The fraction of stored energy recovered in the process of boiling water in a porous volume of rock depends on the amount and pressure of the produced steam, which in turn are determined by the porosity and the initial temperature of the system. The fraction of stored energy recovered for the sweep process is a function of the movement of the cold temperature front. 
Nogucki, T. and others. "Some Theoretical Considerations on Hydrothermal Systems Due to Cracks." U.N. Symposizm on the Development and Utilization of Geothermal Resources. Pisa, Italy, 22 September1 October 1970: 655-661.

Porous Miocene sediments appear to form the major production reservoir at the Otake geothermal field. This is overlain by volcanic cap rock which is highly altered at shallow depths. The permeability of this formation is mainly due to cracks and fractures. : The above system was studied by assuming a horizontal hot water reservoir of infinite extent with associated cracks and production wells in various combinations. The velocity distribution, equipotential surface and stream function for each case are calculated and presented graphically.

Norton, D. and Knight, J. "A Preliminary Analysis of the Coso Geothermal System." Exploration Criteria for Low Permeability Geothermal Resources, Progress Report, June 1975 -March 1977, University of Arizona, Tucson, (1977): 27-53.

An analysis of the Coso geothermal system is presented. A hypothetical geological cross section which includes a thermal energy source is outlined on the basis of synthesized geological, geophysical and geochemical data. Initial and boundary conditions are defined and the problem solved using a two-dimensional finite difference approximation. The analysis suggests the Coso system may contain regions of sufficient permeability and energy content to permit direct production and some other high energy content zones which may require in situ fracturing prior to production. Other observations are reported concerning convective and conductive heat flux and surface energy distribution. In addition, exploration data from the Long Valley thermal system is synthesized, and a geological cross section suitable for defining numerical model parameters is outlined.

Pinder, George F. "The Princeton Geothermal Energy Research Program." Workshop on Geothermal Reservoir Engineering. Stanford. (Stanford University Press, 1975): 199-200.

The geothermal research program at Princeton University is outlined. Topics under study include:

- An extension of the single-phase two-dimensional Galerkin finite element model of the Wairakei reservoir to three dimensions.

- Development of a model for two-phase steam-water systems.

- Development of a model to describe subsidence based on viscoelastic properties of rocks.

- Verification of the conservation equations by applying volume averaging techniques to the continuum equations.

- Simulation of the thermal properties of combined fracturepore systems into which cold water is being injected. 
Prats, M. "Effect of Horizontal Fluid Flow on Thermaliy Induced Convection Currents in Porous Mediums." Jounnal of Geophysical Research 71 (1966): 4835-4838.

Free convection in a porous medium due to a positive temperature with depth gradient was studied using a linear perturbation analys is in the region of the onset of instability. The critical Rayleigh number characterizing the onset of instability is independent of the magnitude of a horizontal fluid current. However, the presence of a horizontal fluid current will cause temperature and velocity fluctuations with time. Recognition of these fluctuations is important to the correct interpretation of geothermal heat flow data. Application of the reservoir simulator to a two-dimensional vertical model of the Wairakei geothermal system has successfully reproduced the total fluid production history over time interval 1953 to 1967. The ground surface subsidence history of the field is examined in terms of the calculated two-phase fluid flow and the local geology.

Pritchett, J. W. "Numerical Calculation of Multiphase Fluid and Heat Flow in Hydrothermal Reservoirs." Workshop on Geothermal Reservoir Engineering. Stanford. (Stanford University Press, 1975): 201-205.

Described here is a multidimensional multiphase finite difference simulator of heat and mass transfer in hydrothermal systems. The flexibility of the code in dealing with one, two, or three dimensional problems for fluid flow in anisotropic porous media with a variety of boundary conditions is discussed. The simulator has been tested using simplified analytical techniques and experimental results for one-dimensional flow through a porous core. Conservation of mass is assured by using density and internal energy as the independent variables.

Pritchett, J. W., Garg, S. K., and Riney, T. D. "Numerical Simulation of the Effects of Reinjection Upon the Performance of a Geopressured Geothermal Reservoir. "Geothermal Resources Council Transactions 1, (7977): 245-247.

Results are presented for the computer simulated performance of a geopressured geothermal reservoir under various exploitation strategies. The reservoir is simplified as a rectangular section of finite thickness with initial uniform fluid and matrix properties and simple no-heat or mass-flow boundary conditions. The produced fluid temperature and methane production are maintained for a much longer period if water is reinjected after use. The production efficiency and life of the reservoir is greatly increased and hence the energy benefit of reinjection is clearly demonstrated. This must be compared wi th the energy cost of pumping brine into the formation which depends on the permeability of the system and details of the surface equipment. 
Ramey, Henry J. Jr. and others. "Geothermal Reservoir Engineering Research." Conference on Research for the Development of Geothermal Energy Resources. 23-25 September 1974: 268-280.

The broad objectives of the geothermal reservoir engineering research at Stanford are: (1) the development of experimental and computational data to evaluate the optimum performance of fracturestimulated geothermal reservoirs; (2) the development of a geothermal reservoir model to evaluate important thermophysical, hydrodynamic, and chemical parameters based on fluid-energy-volume balances as part of standard reservoir engineering practice; and (3) the construction of a laboratory model of an explosion-produced chimney to obtain experimental data on the processes of in-place boiling, moving flash fronts, and two-phase flow in porous and fractured hydrothermal reservoirs.

Current research in support of the above objectives is presented. The chimney model is briefly described and preliminary results on the heat transfer characteristics of the model are outlined. The linear bench-scale model is described and a test series including single-phase and two-phase nonisothermal flow is outlined. In addition, a study on the use of naturally occurring radon as a diagnostic tool for describing the performance of geothermal reservoirs is outlined. Mathematical models are also being developed for comparison with the experimental results in order to identify the principal physical parameters relevant to geothermal reservoir performance.

Ramey, Henry J. Jr. and London, A. Louis. "Stimulation and Reservoir Engineering of Geothermal Resources." Stanford Geothermal Program, TR-8. (Stanford University Press, 1975).

Experimental efforts were directed towards understanding highly fractured rock/water systems. The objective was the study of energy extraction from fractured rock. Fluid recharge was limited so that energy addition by fluid recharge was less than energy extraction from the rock media. The design and construction of a laboratory model for a stimulated geothermal reservoir (the chimney model) was part of the work. Efforts were made to analytically model and predict the observed transient behavior.

Tests were run with two different rocks - gabbro and granitic rock. A total of 29 runs was made with the chimney model. Early runs of this test series had no recharge while most of the later runs had recharge of either cool or hot water, or hot water at nearly constant inlet temperature.

Ribando, R. J. "Natural Convection in a Porous Medium: Effects of Confinement, Variable Permeability and Thermal Boundary Conditions." American Society of Mechanical Engineers Paper No. 76-HT-Y. New York (1976).

Two-dimensional calculations are reported for steady-state natural convection of a fluid in a porous, horizontal layer heated from below. 
Finite difference techniques are used to solve the transport equations for stream function and temperature. Effects of the following parameters are examined: rigid (impermeable) and constant-pressure (permeable) upper boundaries; isothermal and uniform heat flux at the lower boundary; and permeabilities which are constant, or which vary with depth to simulate compaction of a porous medium or property variations of real fluids within the medium. Steady state results are presented for the heat flux distribution on the upper surface, as well as for flow and temperature fields in the interior.

Rinehart, John S. Alterations of Flow Characteristics Within Geothermal Areas by Tidal Forces. (National Technical Information Publication PB-247-190, Springfield, 1975).

Tidal forces produce changes in the state of stress and strain within the earth. This paper studies the effect of tidal forces on the flow characteristics of geothermal systems. Although the tidal stresses are smal1, the longer period tides could produce significant dimensional changes in the separation of fracture surfaces and the size of flow channels, and could appreciably influence the distribution of pore pressure.

Robinson, John. Modeling of Convective Flows. (Department of Scientific and Industrial Research, Applied Mathematics Division Report No. 35, Wellington, New Zealand, 1975).

Modeling of natural convective flows is descriptively summarized. Contributions to the literature are cited and include examples of analytical, numerical and experimental approaches. The basic physical principles which determine the nature of thermal convection are outlined. The change in character of the convective motions from steady two-dimensional roles to oscillatory convection with increasing Rayleigh number is demonstrated, and the effects of cell geometry and boundary conditions on these motions are discussed.

Estimates of the oscillation time constant for Wairakei suggests that for practical purposes the field can be considered to be steady state. It is further submitted that the expected amount of recharge that could be induced by production is small compared with the initial natural flow, and, as a result, the exploitation of the field should be considered a mining operation.

Robinson, Ronald. A Study of the Effects of Various Reservoir Parameters on the Performanoe of Geothermal Reservoirs. Ph.D. dissertation, Texas A \& M University (1974).

A one-dimensional numerical model was developed to simulate geothermal reservoirs. The effects of gravity, capillary pressure, phase change, heat conduction, and heat convection are considered in the model. 
Continuity of species and energy across a boiling front is handled by the use of a buffer cell. It was found that the total amount of heat which can be produced from a geothermal. reservoir is limited and that extraction efficiency is enhanced if the reservoir pressure is reduced to allow water to flash to steam; also the recharge of heat to the reservoir by conduction or from water influx resulted in little additional heat recovery. Reinjection of water into a liquid dominated geotherma 1 reservoir is shown to have little effect on the ultimate heat recovery except in the case of a high porosity reservoir.

Rogers, F. T. Jr. "Convection Currents in Porous Media. III. Extended Theory of the Critical Gradient." Journal of Applied Physics 21 (1950): $1177-1179$.

The theory of the critical gradient for onset of thermal convection in a fluid entrapped in a porous medium is extended to allow for the exponential dependence of viscosity upon temperature and for nonl inear vertical temperature distributions which characterize transients in heat flow. Although there are approximations in the mathematical treatment, the extended theory agrees with experimental data. New experimental data are reported for the critical gradient, obtained largely with silicone fluids in unconsolidated sands.

Rogers, F. T. Jr. and Schilberg, L. E. "Convection Currents in Porous Media. IV. Remarks on the Theory." Journal of Applied Physics 22 (1951): 1476-1479.

The one-dimensional differential equations for critical stability of a fluid in a porous medium and heated from below are solved by using a potential function definition which allows for density variations with temperature. A single formula is obtained for the thermal environment at critical stability allowing for temperature dependence of viscosity and nonlinear temperature distributions. New experiments are reported in which initial convection has been detected by visual, thermal, and radioactive-trace means. Experimental thermal gradients for critical stability deviated from values predicted by theory by the following average amounts: visual, $150 \%$, thermal, $132 \%$, radioisotopic, $11 \%$.

Sammis, Charles G. "Numerical Modeling of Hydrothermal Reactions in Geothermal Reservoirs." Workshop on Geothermal Reservoir Engineering. Stanford. (Stanford University Press, 1975): 240-241

Water-rock chemistry can be expected to affect the evolution of a reservoir in at least three ways: (a) the thermodynamic properties of water are affected by the dissolved solids, (b) the porosity and permeability change with time due to dissolving, precipitation and volume changes associated with alteration, and $(c)$ the heat of reaction may contribute directly to the energy production. 
This paper summarizes the important hydrothermal reactions in a granitic source rock and discusses the evolution of chemical energy due to the dissolving, precipitation, and alteration reactions common in geothermal systems. It is proposed that changes in porosity and permeability associated with both dissolving and alteration reactions can be easily incorporated into the standard finite difference schemes commonly used in numerical reservoir modeling.

Scherer, Charles R. "On the Optimal Rate of Geothermal Energy Extraction." Workshop on Geothermal Reservoir Engineering. Stanford. (Stanford University Press, 1975): 161-166.

The simple single phase steady flow model of a hydrothermal production-injection system developed by Gringarten and Sauty is used to develop economic models for the optimal rate of heat extraction." The investment in well, piping, conversion equipment, and operating costs are considered, and optimal extraction policies are derived for the cases of steady and non-steady fluid withdrawal rates. The quantitative economic models are derived, and it is shown that iterative solutions can be obtained which maximize the discounted net revenues of the system.

Schrock, V. E. and Laird, A. D. "Physical Modeling of Combined Forced and Natural Convection in Wet Geothermal Formations." Joumal of Heat Transfer 98 (1976): 213-220.

Some general considerations for physical modeling are presented as well as the results obtained from a laboratory model having two wells to simulate a geothermal energy extraction loop. The governing dimensionless parameters are deduced from the macroscopic partial differential equations expressing conservation of mass, momentum and energy in a porous medium. The experiments were carried out using an insulated rectangular box heated from below, with temperature histories being recorded for a number of different production injection rates. Significant three-dimensional effects were recorded making numerical comparison difficult. It was concluded that although the technique showed some promise, the experiments were cumbersome to set up and time consuming to perform.

Shen, H. W. "A Hele-Shaw Model of Heat Convection in Porous Media Under Geothermal Conditions." Workahop on Geothermal Reservoir Engineering. Stanford. (Stanford University Press, 1975): 213-218.

Experimental studies of two-dimensional free convection using a Hele-Shaw cell are described. The experiments were designed to investigate heat and mass flow characteristics under variable fluid viscosity conditions at high Rayleigh numbers. These conditions are difficult to analyze by mathematical techniques. An experimental relationship is established between the Nusselt number and Rayleigh number, and the deep groundwater motion is visualized for a model based on physical character- 
istics typical of the Taupo volcanic zone in New Zealand. The results are consistent with the hypothesis of an initial phase of volcanism generated convecting water system still present after 1.5 million years.

Sinnokrot, Ali A. "Effect of Temperature Level Upon Capillary Pressure Curves." Society of Petroleum Engineers Journal 11 (1971): 13-22.

The paper concentrates on a systematic study of temperature effects upon capillary pressure. Equipment was constructed to permit capillary pressure measurements at temperatures ranging from room temperature to about $350^{\circ} \mathrm{F}$. For three consolidated sandstones and one limestone sample, drainage and imbibition capillary pressure curves were measured at three or four temperature levels from $70^{\circ}$ to $325^{\circ} \mathrm{F}$. Fluids used were a filtered white oil and distilled water. The practical irreducible water saturation for the sandstone samples increased significantly as temperature was raised. However, the hysteres is between drainage and imbibition cycles decreased as temperature increased and was almost absent at $300^{\circ} \mathrm{F}$. All capillary pressure-saturation curves for limestones for various isotherms were found to lie within the envelope of the room-temperaure drainage and imbibition curves.

Somerton, W. H. "Effects of Temperature and Stress on Fluid Flow and Storage Capacity of Porous Rocks." Site Characterization: Proceedings 17th U.S. Symposizm on Rock Mechanics. Snowbird, Utah, 25-27 August 1976: 2A2-1 to 2A2-8.

Measurements of sonic velocities, bulk and matrix compressibilities and thermal conductivities were made on a group of outcrop sandstones and a group of siltstone cores obtained from wells drilled in the Imperial Vailey, California. The measurements were made at temperatures to $200^{\circ} \mathrm{C}$ and pressure to 16,000 psi. Sonic velocities decreased with increased temperature and increased with increased pressure and with liquid saturation. Both bulk and matrix compressibilities increased with increased temperature. Thermal conductivities were of the expected order of magnitude for sandstones and siltstones of the type tested. Vaporization-condensation reactions caused a more than two-fold increase in apparent thermal conductivity for the higher permeability sandstones but had essentially no effect on the conductivities of the low permeability siltstones. The results are discussed relative to their application in high temperature underground reservoir studies.

Sondergeld, Carl H. and Turcotte, D. L. "An Experimental Study of TwoPhase Convection in a Porous Medium with Applications to Geological Problems." Joumal of Geophysical Researeh 82 (1977): 2045-2053.

Two-phase thermal convection in a porous medium was carried out in a sandbox. The experimental study was initiated by heating the watersaturated porous layer from below and cooling from above. When the lower layer reached $100^{\circ} \mathrm{C}$, a two-phase steam-water zone was developed. Study 
of the variable parameters, such as the thickness of the porous layer and the heat flux, indicated that there could exist a stable form of two-phase convection in porous media. The two-phase region was of variable geometry and had a clear non-horizontal steam-water interface with the overlying water zone. Occurrences of the phase change initiated convective instabilities. At high heat fluxes a dry steam zone developed in which the temperature exceeded $110^{\circ} \mathrm{C}$. Location of the dry steam zone was close to the base plate with the wet steam above. The experimental data were analyzed with a one-dimensional heat and mass transfer model.

Sorey, M. L. "Numerical Modeling of Geothermal Systems," Geological Society of America Abstracts with Programs 6 (1974): 962.

Numerical solutions to the equations describing the transport of heat and water in porous media are being used to study steady state conditions in areas of potential geothermal development. Using preliminary data on temperature distributions, geochemistry, and hydrology, various models for the natural conditions in these systems can be tested and additional data requirements can be evaluated.

A computer program has been developed to treat problems in one, two or three dimensions involving simple or complex geometries. Model application in the Long Valley. California, area includes studies of heat transfer in an upflowing hot spring system and a rechargingdischarging reservoir. Studies of natural (cellular) convection in porous media have also been made.

Sorey, M. L. Numerical Modeling of Liquid Geothermal Systems.: Ph.D. dissertation, University of Callfornia, Berkeley (1976).

A mathematical model is developed for single-phase natural convection in an isotropic porous medium. The equations are solved in two and three dimensions using an integrated finite difference technique. The resulting computer code is used to study the onset of convection at critical Rayleigh numbers and cellular convection in a hypothetical "base" model. The resulting Rayleigh number/Nusselt number relationships are compared with other published experimental and numerical results. The model is then extended to study the effect of various temperature-dependent fiuid parameters such as viscosity and compressibility at the onset and magnitude of convection. The effects of various boundary conditions and surface hot spring configurations on the thermal characteristics of the reservoir and surroundings were also studied. The surface heat flow profile is found to depend on the nature of the hot spring conduit and the structural and hydrological conditions. This can be used to define the probable subsurface environment and subsequently help direct further exploratory work when assessing geothermal systems. 
Sorey, M. L. "Research on Numerical Modeling of Liquid Geothermal

Systems." Workshop on Geothermal Reservoir Engineering. Stanford.

(Stanford University Press, 1975): 225-231.

The conservation equations are solved numerically using an integrated finite difference technique to model slightly compressible fluid flow and heat transfer in multidimensional porous media. Nusselt number/Rayleigh number plots for circulatory convection compare favorably with numerical and experimental results from other authors.

The model is used to study the onset of convection and reveals that the critical Rayleigh number is substantially reduced if variations in fluid viscosity and expansivity with temperature are included.

Heat and mass transfer associated with hot spring systems is analyzed to determine the amount of heat lost by conduction and convection to the rocks surrounding the spring conduit. Both cylindrical and plane conduit models surrounded by permeable and impermeable rock are considered. It is concluded that the surface temperature distribution around a hot spring area could outline some of the structural features of the underground system and assist in evaluating methods for further exploration. Ultimately the imodel can be used to define an optimum reservoir exploitation strategy.

Spencer, James W. Jr. "Effects of Pressure, Temperature, and Pore Water on Velocities in Westerly Granite." Joumal of Geophysical Research 81 (1976): 899-904.

Sonic velocities were measured with the ultrasonic pulse transmission method in an internally heated pressure vessel. At a given temperature, confining pressure increase results in an increase in wave velocity of dry samples. At a given confining pressure, increased temperature results in a reduction in the shear wave velocity. Poisson's ratio of dry rock increases with the combined effects of temperature and pressure. At low pressures, with increasing temperature, saturated samples show a continuous decrease in the bulk modulus. Shear modulus is affected oniy by the temperature of the crystalline matrix. Thus, the low compressional wave velocity and nearly constant shear wave velocity cause a decrease in the Poisson's ratio. Under the same pressure and temperature conditions, 'samples with high pore fluid pressure result in a decrease in shear wave velocity, while compressional wave velocity is less affected. Poisson's ratio increased. Time-dependent compressional velocities were attained in the laboratory samples by a solution of quartz in supercritical pore water.

Straus, Joe M. "Large Amplitude Convection in Porous Media." Journal of Fluid Mechanics 64 (1974): 51-63.

The properties of convection flow driven by an adverse temperature gradient in a fluid-filled porous medium are investigated. The Galerkin technique is used to treat the steady-state two-dimensional problem for 
Rayleigh numbers as large as ten times the critical value. The stability of the finite amplitude two-dimensional solutions is treated and the dependence of Nusselt number on Rayleigh number determined. At a given value of the Rayleigh number, stable two-dimensional flow is possible for a finite band of horizontal wave numbers as long as the Rayleigh number is small enough. For Rayleigh numbers larger than about 380, however, no two-dimensional solutions are stable. Good agreement with experimental results is demonstrated.

Straus, Joe M. and Schubert, G. "Thermal Convection of Water in a Porous Medium: Effects of Temperature- and Pressure-Dependent Thermodynamic and Transport Properties." Journal of Geophysical Research 82 (1977): 325-333.

Natural convection of water in thick geothermal layers, across which there are temperature differences as large as $345 \mathrm{~K}$ and pressure differences as great as $1 \mathrm{kbar}$, is investigated. Complete account is taken of the variable thermodynamic and transport properties of water as well as of non-Bouss inesq effects. The increase of thermal expansivity with temperature and the decrease of viscosity are primarily responsible for the enhanced instability to convection of a water-saturated porous layer compared with a porous layer saturated wi th a Boussinesq fluid having the constant properties of surface water. The critical Rayleigh number, critical wave number, and streamline and isotherm patterns are determined at the onset of convection for a range of temperature gradients in layers as thick as $10 \mathrm{~km}$. The critical surface Rayleigh number is reduced by as much as a factor of 31 below the value of $4 \pi^{2}$, appropriate for a constant property Boussinesq fluid. The horizontal scale of convection is somewhat reduced, and the flow is concentrated toward the bottom of the porous layer by effects of variable expansivity and viscosity.

Takahashi, Patrick K. and Chen, Bi11., "Geothermal Reservoir Engineering." Geothermat Energy Magazine 3 (1975): 243-257.

The activities which can be defined by the title Geothermal Reservoir Engineering are discussed. In particular, a reservoir engineering program is outlined and the various topics which make up this program including conceptual modeling, well testing, physical and mathematical modeling are discussed. Significant contributions in each of the areas outlined are referenced and briefly summarized.

Tansev, Erdal 0. "Modeling the Heber Geothermal Reservoir." Geothermat Resources Council Iransactions 2 (Part 2, 1978): 645-648.

A single-phase numerical model of the Heber Geothermal Field 'is presented in which the reservoir is conceptualized as a roughly circular shaped stack of interbedded sandstones and shales. Production of the reservoir is maintained at the level necessary to service a 250 MWe power station. Brine is injected after use at the boundary of the system 
at a temperature of $200^{\circ} \mathrm{F}$ and production is from the center of the system which is divided into radial slices. It is assumed the fluid flows as a homogeneous two-phase mixture.

Some gravity segregation of steam and water, which hastened the formation of a superheated region in the upper portion of the reservoir, is noted in the cross sectional simulations. This effect is countered by the generation of two-phase regions below a continuous water layer due to the presence of deep producing wells. In addition, it is noted that superheated regions formed more quickly for reservoirs of low porosity and permeability.

Tezcan, A. Kenan. "Dry Steam Possibilities in Saraykov-Kizildere Geothermal Field, Turkey." Second U.N. Symposium on the Development and Use of Geothermal Resources. San Francisco, 20-29 May 1975: 1805-1813.

A conceptual model of the Kizildere geothermal field, Turkey, is proposed. Topographic conditions, geothermal gradient surveys, and deep drilling results are used to formulate the model. It appears the reservoir could consist of a steam cap trapped beneath a low permeability confining layer. This cap is produced from a boiling zone with the temperature of water below this zone roughily following the boiling point depth curve. It is concluded that the field could be successfully produced from the dry steam zone.

Toronyi, R. and Ali, S. M. Farouq. "Two-Phase, Two-Dimensional Simulation of a Geothermal Reservoir." Society of Petroleum Engineers Paper No. 5521. Dallas (1977).

Two-dimensional areal and vertical cross-sectional simulations were carried out on the heat and mass flow in a two-phase geothermal reservoir with isotropic permeability and no heat or mass flow boundary conditions. The conservation equations were solved numerically using finite difference approximations for the reservoir and wellbore system. Passage of steam through already dried rock produces superheating. After an initial vaporization of liquid water in the reservoir, the decrease in pressure produces increased boiling below the deep water table. With heavy exploitation, boiling extends deeper into hotter rock and the temperature of the steam increases.

Truesde11, A. H. "Production of Superheated Steam from Vapor-Dominated Geothermal Reservoirs." Geothermics 2 (Number 3-4, 1973): 154-173.

Conceptual models for vapor-dominated geothermal systems such as The Geysers, Larderello and Matsukawa are discussed. Production data and the thermodynamic properties of steam and water are used to examine. the heat and mass transport phenomena which occur in these systems.

It is concluded that these systems initially consist of a water and 
steam filled reservoir, a water-saturated cap rock, and a water or brinesaturated deep reservoir below a water table. Most liquid water in all parts of the system is relatively immobilized in small pores and crevices; steam dominates the large fractures and voids of the reservoir and is the continuous, pressure-controlling phase.

Tsang, Chin Fu, Lippman, M. J., and Witherspoon, P. A. "Production and Reinjection in Geothermal Reservoirs." Geothermal Resources Council Transactions 1 (1977): 301-303.

Recent work at the Lawrence Berkeley Laboratory in the study of fluid production and injection in liquid-dominated geothermal reservoirs is reviewed. Two major approaches, a semi-analytical method and a three-dimensional numerical model are described. Five examples are discussed: (1) a production-injection doublet, (2) a system with nine production and five injection wells, (3) temperature recovery after a period of injection, (4) consolidation during fluid withdrawal, and (5) transient pressure responses during production-injection-production procedures.

Weres, Oleh and Schroeder, Ron C. Documentation for Progran OGRE. (Lawrence Berkeley Laboratory Report LBL-7060, 1978).

A listing and brief description of a computer program designed to generate discrete grids for integrated finite difference computer programs is presented. The program is specifically intended for large scale two or three dimensional reservoir simulation. The operation of the program is demonstrated on a simple sample problem. A grid structure for the Larderello geothermal field in Italy is also generated using the program.

Wooding, R. A. "An Approach to Geothermal Development." Workshop on Geothermal Reservoir Engineering. Stanford. (Stanford University Press, 1975): 216-219.

This paper outlines the need for a broad data bank and how past production histories can help in getting a view of how the future is going to look. Performance modeling of geothermal fields has been proposed and applied to Broadlands field, New Zealand, with experience gained from Wairakel field, New Zealand. Some of the pitfalls encountered in doing such performance modeling are outlined. The importance of geology, geophysics, petrology, geochemistry, hydrology, structural models and tectonics on the exploitation strategy and future performance is acknowledged. 
Wooding, R. A. "An Experiment on Free Thermal Convection of Water in Saturated Permeable Material." Joumal of Fluid Mechanics 3 (1957): 582-600.

A small scale experimental model was constructed to study the steadystate slow free convection of water in saturated sand. The convection field was confined between coaxial cylinders maintained at constant temperature differences $\left(T_{1}-T_{0}\right)$ of (1) $18.40^{\circ} \mathrm{K},(2) 32.70^{\circ} \mathrm{K}$, and (3) $46.68^{\circ} \mathrm{K}$, where measurements of the temperature distribution within the convection space were obtained. The least square method was used to fit the theoretical temperature values predicted from perturbation theory. A good agreement between theoretical and experimental results was obtained. Separate determination of convection parameter or modified Rayleigh number, temperature coefficient of thermal conductivity of the sand-water mixture, and coefficient of radiation loss at the upper insulated boundary by direct physical measurement were in agreement with the least square estimates.

Wooding, R. A. "Free Convection of Fluid in a Vertical Tube Filled with Porous Material." Journal of Fluid Mechanics 13 (1962): 129-144.

Study is directed to the free convection of a fluid in a vertical tube filled with porous material. The problem of an unstable fluid overturning in the vertical tube is considered. It is shown that the fluid can experience a pseudo-inertial effect, in which variations in density across the tube exhibit properties analogous to variations of momentum in an inertial flow. Changes in the mean density of the fluid can be propagated as discontinuities, and for discontinuities of finite amplitude, two jump conditions are derived, one of which is identified to involve an undetermined parameter.

Wooding, R. A. "Instability of a Viscous Liquid of Variable Density in a Vertical Hele-Shaw Cell." Joumal of Fluid Mechanics 7 (1960): 501-515.

Approximation equations of motion, continuity, and mass transport are required to calculate approximate stability criteria for a viscous liquid of variable density moving very slowly between vertical and impermeable parallel planes. The results are applicable to the problem of the stability of a viscous liquid of variable density to two-dimensional disturbance in a porous medium. The study also provides an exact stability analysis for the liquid between parallel planes. An asymptotic expansion is derived for the critical Rayleigh number at neutral stability in a long vertical channel, or duct, with a thin rectangular section. The critical Rayleigh number for a long vertical channel of rectangular cross-section is determined experimentally by comparing the density gradient of the liquid in the channel at neutral stability with the corresponding density gradient in a vertical capillary tube. 
Wooding, R. A. "Rayleigh Instability of a Thermal Boundary Layer in Flow Through a Porous Medium." Joumal of Fluid Mechanics 9 (1960): 183-192.

It is supposed that heated liquid is rising by natural convection very slowly through a semi-infinite porous medium towards the permeable horizontal surface, where it mixes with a layer of cool overlying fluid. In the steady state, a thermal boundary layer of exponential form exists in the medium. It is shown that the layer is stable provided the Rayleigh number for the system does not exceed a critical positive value and that the wave-number of the critical neutral disturbance is finite. The stability properties of the layer are explained qualitatively from physical considerations.

Wooding, R. A. "The Stability of a Viscous Liquid in a Vertical Tube Containing Porous Material." Proceedings of the Royal Society of London 252 (1959): 120-134.

The density gradient at neutral stability was compared experimentally with Taylor's value for the corresponding density gradient in a vertical capillary tube. For a porous medium consisting of randomly packed glass spheres of mean diameter of about $0.2 \mathrm{~mm}$ and $36.5 \%$ porosity $(\phi)$, it was found that the two results were consistent providing the ratio $k / D \emptyset=$ 0.633 , where $k$ is the diffusivity of the solution and $D$ is the molecular diffusivity of the solute when the porous medium is absent. Since this dimensionless ratio is a property of the porous material alone, diffusion measurements were employed to determine the ratio directly.

Wooding, R. A. "Steady-State Free Thermal Convection of Liquid in a Saturated Permeable Medium." Journal of Fluid Mechanics 2 (1957): 273-285.

The partial differential equations which describe steady flow of fluid in saturated homogeneous permeable solid material under nonisothermal conditions are stated. From these are derived the equations for flow of liquid (in particular, water) using suitable approximations and making use of empirical laws for constitutive properties when necessary. It is then postulated that the only mass moving forces present are those due to thermal expansion effects. Free convection results. An approximate solution of the equations is attemped for plane flow by means of classical perturbation methods, the temperature and stream-function variables being represented by power series in a convection parameter proportional to the Rayleigh number.

A numerical example of the method is given with boundary conditions based on a geothermal area at Wairakel, New Zealand. The results show features which are in fair agreement with temperature measurements made in the area. 


\subsection{Exploitation Strategies}

Early geothermal development concentrated more on the immediate problems of a new technology than on the consequences of long term exploitation of a finite resource. An example of this is at Wairakei, New Zealand, where the high pressure turbines in the power station were designed to accommodate steam at near maximum deliverable pressure of the reservoir. The reservoir, however, has drawn down considerably during production, and it has become increasingly difficult to maintain generation in the high pressure sets.

It is essential to match the energy conversion process to the reservoir and ensure the two will remain compatible over the life of the development. Bolton [1973] outlines some of the factors which must be considered in assessing the power potential of a reservoir. In addition to the obvious reservoir production related factors, he stresses the importance of considering the indirect effects of long term development such as subsidence, pollution, corrosion and deposition. Nathenson [1975] studied the various factors which affect the fraction of stored heat that can be recovered by various strategies and concludes that reservoir production can be optimized by correct choice of the process. James [1965] compared the power life of a hydrothermal system for the two strategies of induced boiling in the formation and subsequent steam draw-off, and compressed water production. He concludes that more rock heat is extracted in the case of boiling. Papadopulos [1975] uses hydrological techniques to define the optimum exploitation strategy for a given geothermal geopressured reservoir. It appears that shale-water influx has a significant effect on reservoir deliverability in this case.

Recent studies show that it is feasible to maintain pressures and enhance the long term deliverability of a reservoir by injecting spent fluid back into the formation. Chasteen [1974] reports that steam condensate has been successfully injected at The Geysers and proposes that similar strategies would be appropriate for the hot water fields in the Imperial Valley, California, and Valles Caldera, New Mexico. Einarsson [1975] reports the results of an extensive injection test at the Ahauchapan geothermal field and submits that since fluid fronts move faster than thermal fronts in a porous medium, significant chemical change in production fluids could be a precursor to thermal breakthrough of injected water.

A single-phase steady-flow model is used by Tsang [1978] to study the effects of various exploitation strategies at Cerro Prieto. It is concluded that water could be injected safely without causing premature quenching of the production formation. Kasameyer [1977] calculates flow and temperature fields for arbitrary production injection strategies at the Salton Sea geothermal field. It is shown that anisotropic flow properties can alter the predicted flow patterns considerably. Tsang [1975] uses a simplified reservoir model to show that a screening well placed between injection and production wells through which fluid is 
rerouted back into the injection well can significantly prolong the time before thermal breakthrough occurs.

Many hot water resources are produced by self-pumping with a boiling. steam-water mixture produced at the well head. To maximize the availability of fluid reaching the well head, the borehole must be designed to pass these fluids with minimum energy loss. Gould [1974] developed a model for a two-phase flow in a wellbore which compared favorably with experimental results. Ridgway [1977] uses established correlations to predict pressure drops in a flowing wellbore. The methods, however, produce inconsistent results; one suggesting an increase in diameter will improve performance, while the other indicates a decrease in diameter.

For fields where natural fluid circulation is small or absent, artificial circulation of a working fluid to extract heat is being considered. A large research effort in this direction is currently being conducted by the Los Alamos Scientific Laboratory at a hot-dry rock resource on the flanks of the Valles Caldera. Tester [1976] describes the fundamentals of this program and outlines the individual areas of importance to research. Sarda [1975] discusses the rock mechanics aspects of creating and maintaining a hydraulic fracture in hot rock. Murphy [1975] calculates the heat transfer from rock to fluid circulating in a hydraulic fracture and submits that rock greater than 40 meters away from the fractured surface is not affected even after ten years of production. McFarland [1976] shows that natural buoyancy forces play a major role in the fluid-flow pattern in a hydraulic fracture and submits that in the absence of short circuiting due to areas of stationary fluid, the rate at which energy can be produced is limited only by the size of the fracture. The additional heat exchange surface created by thermal contraction-induced secondary cracks is studied by Hsu [1978].

Explosively enhanced permeability has also been considered. Aladiev [1975] submits that the amount of energy that can be extracted from an explosively generated zone of high permeability is determined by the degree of natural convection induced. Diadkin [1976] calculates the size of zone created by explosive stimulation and considers suitable economic sizes and depths. McKee [1975] considers the propagation of an explosion from a wellbore in two stages. The first stage comprises a stress wave which initiates fractures. These are then propagated by a pressure wave of expanding gas.

It is evident that considerable research is being directed toward enhancing the productivity of geothermal reservoirs and increasing the Tongevity of systems through optimized production management strategies. The practical results of this work are now being realized as more geothermal systems are developed. 
Aamodt, R. Lee. Artificial Geothermal Reservoirs in Hot Volcanic Rock. (Los Alamos Scientific Laboratory Report LA-UR-73-1995, 1973).

Various methods of extracting energy from hot dry rock are discussed. The creation of a significant heat exchange surface is emphasized, and the rock mechanic principles involved in hydraulic fracturing are outilined. The advantages of multiple hydraulic fractures and themal stress cracking to increase the heat exchange area are also discussed.

Aladiev, I. T. and others. "Heat and Mass Transfer Processes in Aquifer Systems with Artificially Increased Fracturing." Second U.N.

Symposium on the Development and Use of Geothermal Resources. San Francisco, 20-29 May 1975: 1529-1535.

A mathematical model of an aquifer system with artificially increased permeability is constructed. The results of experiments on thermal disturbance propagation carried out in a groundwater aquifer with explosively enhanced permeability confirm the predictions given by the mathematical model. It appears that the characteristics of natural convection in the zone of high permeability will determine the degree of heat transfer and exploitation potential of the system.

Bodvarsson, Gunnar. "Evaluation of Geothermal Prospects and the Objectives of Geothermal Exploration." Geoexploration 8 (1970): 7-17.

The extraction of heat from a geothermal reservoir is discussed. The necessary volume, temperature, permeability and hydrological features of the reservoir are outlined on the basis of power cycle requirements. The accessibility of the reservoir is emphasized, and the practical importance of moderate chemical content of the recovered waters is stressed.

Bolton, R. S. "Management of a Geothermal Field." UNESCO Earth Sciences 12 (1973): 175-184.

The important factors in managing a geothermal development are discussed. The methods for estimating the energy potential of a geothermal field are qualitatively outlined. Some significant factors in assessing this potential include: estimates of natural heat flow and stored heat, the anticipated method of exploitation, and the method of disposal. The primary effects of exploitation, such as well output characteristics, pressure and temperature drawdown, and cumulative production history are identified and examples of these factors are given for Wairakei, New Zealand, Larderello, Italy, and Laugarnes, Iceland. Important secondary effects of exploitation are also discussed. These include subsidence, chemical and thermal pollution, chemical deposition and corrosion. It is noted that the relative importance of these factors will vary from field to field. Finally the importance of recording field performance is stressed. This includes well head and downhole measurements and monitoring of immediate area topography and natural thermal features. 
Budd, Chester. F. Jr. "Steam Production at The Geysers Geotherma1 Field." In Geothermal Energy: Resources, Production, Stimulation (Chapter 6). Edited by Paul Kruger and Carel Otte. (Stanford University Press, 1973).

The reservoir performance of The Geysers geothermal field is discussed. Techniques adapted from the 011 and gas industry have been used to predict the characteristics of the reservoir and producing wells. The importance of determining these characteristics before choosing a specific power station design and of monitoring the changes in reservoir characteristics with production is stressed. In view of this, pressure drawdown, buildup, and flow tests are carried out, interference between wells is assessed, and production is monitored continuously while the wells are in service.

Calamai, A. and others. "A Reinjection Experiment in Vico 1 We11." Geothermics 2 (Numbers 3-4, 1973): 117-118.

Injection tests near the Larderello and Mt. Amiata geothermal areas were carried out. Cold water was injected at various rates by gravity flow. The chemistry of formation fluids, hole pressure, temperature, and microseismicity in the immediate area were monitored in order to detect injection induced variations. No increased seismic activity was recorded.

Campbe11. Thomas A. "Analys is of Geothermal Well Pumping." Geothermaz Resource Counoil Transactions 2 (Part 1, 1978): 63-66.

A system study of geothermal well pumping includes the evaluation of the problems in determining the net power output of pumped wells for various productivity indices defined as flowrate per unit drawdown. The principle parameters needed are presented, and the present value income of two common well casing programs are compared.

Chasteen, A. J. "Geotherma1 Steam Condensate Reinjection." Conference on Research for the Development of Geothermal Energy Resources. 23-25 September 1974: 340-344.

Steam condensate at The Geysers has been reinjected into the steam reservoir since 1968. A total of $3,150,000,000$ gallons of condensate has been reinjected with no noticeable effect on the adjacent producing wells. Currently, 3,700,000 gallons/day from $412 \mathrm{MW}$ of installed capacity are being injected into five wells. Reinjection has also proven to be a satisfactory method of disposing of geothermal condensate at Imperial Valley, Callfornia, and at Valles Caldera, New Mexico. 
Chen, Che Jen. "Man-made Geothermal Energy Source." Nuclear Instruments and Methods 150 (1978): 365-366.

The power available from an underground thermal nuclear fusion explosion is calculated. The system is conceptualized as a spherical chamber of magma created by a fusion explosion surrounded by a heat exchange system which circulates heat to the surface. The dimensions of the chamber and life of the system are estimated for a number of working fluid temperatures.

Diadkin, Y. D. and Pariisky, Y. M. "Theoretical and Experimental Grounds for Utilization of Dry Rock Geothermal Resources in the Mining Industry." Second U.N. Symposium on the Development and Use of Geothermar Resources. San Francisco, 20-29 May 1975: 1609-1618.

Fractured zone sizes for the explosive stimulation of geothermal resources and their dependence on the physical properties of rock, depth, and weight of explosive charge is based on laboratory test results and analys is of available data on underground nuclear explosions. A thermodynamic method of calculation of the number of fractures and rock permeability is developed, taking into consideration experimental data on the energy consumed in forming new surfaces. A coupled problem on nonstationary heat exchange and nonisothermal water filtration in an underground boiler is solved by means of the approximate finite-element method. Analys is of the circulation systems economic-mathematical model confirms their efficiency at a well depth of no more than $4 \mathrm{~km}$.

Einarsson, Sveinn S., Vides, Alberto R., and Cuellar, Gustavo. "Disposal of Geothermal Waste Water by Reinjection." Second U.N. Symposizm on the Development and Use of Geothermal Resources. San Francisco, 20-29 May 1975: 1349-1362.

Reinjection tests carried out at the Ahuachapan geothermal field are discussed. Deposition problems were alleviated by maintaining the temperature above the silica saturation level. The cooling effect in the vicinity of the well was monitored and shown to rapidly equilibrate with the injected water temperature. Chemical breakthrough of injected water into production wells is a precursor to thermal breakthrough and regular monitoring of production well fluids is recommended to identify major chemistry changes. It is concluded that reinjection is a technically feasible and potentially useful strategy for disposing of waste fluids and for increasing the long-term productivity of a geothermal field.

Gould, Thomas L. "Vertical Two-Phase Steam-Water Flow in Geothermal Wells." Journal of Petroleum Technology 26 (1974): 833-842.

Equations for two-phase steam-water flow in a wellbore are presented. The interactions of heat transfer, fluid flow, phase change, and flow regime are studied. Several correlations for vertical two-phase flow 
are compared with field data for wells discharging steam-water mixtures. A combination Griffith and Wallis/Hagedorn and Brown/Turner Ros model is shown to give the most consistent results. It is concluded that calculations of the flowing pressure and temperature drop can have important applications in well design as well as for the assessment of well deliverability.

Hanson, Jonathan M. "Predicting Production Temperature Using Tracer Methods." Geothermal Resources Council Transactions 2 (Part 1, 1978): 247-258.

The paper presents a simple reservoir model in which heat transfer is due to convection only, showing that the length of time required for an injected fluid element to heat to a given temperature is independent of the streamline path taken by the fluid element. This suggests that a knowledge of the distribution of fluid residence time between injection and production wells is sufficient to estimate the production temperature history for reservoirs for which the model is applicable.

The paper also proposes the use of superposition to evaluate the distribution of fluid resident time in multiple well situations. A general expression for the production temperature is obtained based on this observation and the assumption that the porosity-thickness product. is known or can be estimated.

Hsu, Y. C. and others. "Engineering Methods for Predicting Productivity and Longevity of Hot-Dry-Rock Geothermal Reservoir in the Presence of Thermal Cracks." Geothermal Resources Coincil Iransactions 2 (Part 1, 1978): 297-298.

This paper presents investigation of a hot-dry rock geothermal reservoir regarding: (1) study of the main hydraulic fracture that can be extended through secondary thermal cracks, and (2) the development of methods for predicting the productivity and longevity of a geothermal reservoir.

The model is based on the conversion of mass, momentum, and energy; solution procedures were developed for estimating the additional heat energy which was extracted from stable secondary thermal cracks in a geothermal energy reservoir. The reservoir assumes the form of a large penny-shaped vertical main, hydraulic fracture (crack).

James, Russe11. "Power-Life of a Hydrothermal System." Second Australasian Conference in Bydraulice and Fluid Mechanics. (1965): 211B233B.

A lumped parameter representation of a sealed finite hot water aquifer is used to show that steam withdrawal from a shallow boiling zone is a more effective production strategy than with water production from below the boiling level. Steam draw-off from the top reduces the 
heat content of the formation as well as that of the water to a marked extent while for hot water draw-off from the bottom of the aquifer, a very large amount of residual heat is left in the formation uncovered by the falling water level. The effect of these modes of draw-off on the chloride ion concentration in the water phase is also studied and compared with the actual changes in the Wairakei aquifer.

Juprasert, Sirisak. "A Numerical Simulator for Flow in Geothermal Welibores." Geothermal Resources Couneil Transactions 1 (1977): 159-161.

A computer program has been developed to simulate the flow of hot water or steam-water mixture in geothermal wellbores. Given the downhole fluid conditions and the geometry of the well, the program calculates the pressure, temperature, enthalpy and quality of the discharge at the we11head or any specified depth in the wellbore. Existing two-phase correlations are used with a heat transfer term determined by back-correlating a fit to obsenved data. The program has been tested successfully for two wells from the East Mesa geothermal field.

Kasameyer, Paul and Thorson, Lewis. "Modeling Thermal and Flow Fronts for Arbitrary Well Arrays." Geothermal Resources Counciz Transactions 1 (1977): 163-165.

Flow and temperature fields are calculated for arbitrary productioninjection strategies at the Salton Sea geothermal field. A computer code has been developed which is based on an analytical solution for pressure distribution to determine the temperature distribution and flow fronts of injected fluid within a single homogeneous, nonleaky, liquid-filled aquifer of uniform thickness and infinite extent. It is shown that anisotropic flow properties can alter the predicted flow patterns considerably.

Kubota, Katsundo. "Reinjection of Geochemical Hot Water at the Otake Geothermil Field." Second U.N. Symposizm on the Devezopment and Use of Geothermal Resources. San Francisco, 20-28 May 1975: 1379-1383.

Since March 1972, geothermal waters have been injected after use at the Otake geotherma.7 field. In addition to providing a convenient method of disposing of the effluent, it is hoped that the injection will provide some recharge to the system and thereby increase the longevity of the field. The location of the injection wells is discussed, which in one case is only 150 meters from a producing we11. Thus far, injection has not influenced the ground water and surrounding hot springs, and there has been no indication of leakage to the surface. In addition, the suspected increase in earthquake activity has not occurred. 
Kuwada, J. T. "Geothermal Reservoir Pressure Requirements for Production." Workshop on Geothermal Reservoir Engineering. Stanford. (Stanford University Press, 1975): 157-160.

The production aspects of reservoir engineering are discussed. For example, well spacing and wellbore design are reservoir-controlled parameters of specific interest to field production management. Degradation of the availability of produced fluids between the reservoir and the process can be reduced by artificially lifting fluids to suppress flashing. This has the added advantage of maintaining the chemical integrity of the fluids and reducing the possibility of scaling in surface equipment.

Laughtin, A. W. and others. "Status of the Los Alamos Experiment to Extract Geothermal Energy from Hot Dry Rock." Geology 4 (1977): 237-240.

Two deep holes (GT-2 and EE-1) have been drilled at the Fenton Hill site in northern New Mexico in support of the Los Alamos Scientific Laboratory's hot dry rock geothermal development project. The first hole, GT-2, has a depth of $2,928 \mathrm{~m}$ and a bottom hole temperature of $197^{\circ} \mathrm{C}$. The second hole, EE-1, has been temporarily halted at a depth of $3,062 \mathrm{~m}$ where the temperature is $205.5^{\circ} \mathrm{C}$. Hydraulic fractures have been created in both holes and fluid communication between the holes was established in October 1975. Circulation experiments are now being conducted between the two holes.

McFarland, R. D. and Murphy, H. D. "Extraction Energy from HydraulicallyFractured Geothermal Reservoirs." Eleventh Intersociety Conversion Engineering Conference. 12-17 September 1976: 828-835.

The governing equations for heat and mass transfer are derived for flow in a vertical hydraulic fracture. The equations are simplified by assuming inertia forces are sma 11 and convection dominates conduction in the crack. Numerical solutions are obtained for non-steady heat transfer from the wall rock to the circulating fluid. Buoyancy is shown to play a major role in the resulting filow streamlines. It is concluded that in the absence of short-circuiting due to areas of stationary fluid, the rate at which energy can be produced is limited only by the size of the fracture. The extent of short-circuiting is determined by the location of the fluid inlet and outlet and buoyancy effects. Thermal contraction will reduce the tendency for short-circuiting to occur.

McKee, C. R. "Predicting Explosion-Generated Permeability Around Geothermal Wells." Workshop on Geothermal Reservoir Engineering. Stanford. (Stanford University Press, 1975): 192-197.

Detonating explosives in a borehole is one technique for stimulating tight geothermal formations. Explosives may also have an application 
where precipitation of solids near the producing well has significantly reduced the permeability around it. . A theory is outlined which considers the propagation of an explosion in two stages. The first stage comprises a stress wave which initiates fractures in the reservoir rock which are then propagated by the pressure of expanding gas. The theory is found to correlate well with existing measurements of permeability enhancement.

Miller, Richard J. "Enhanced Recovery Reservoir Engineering Aspects of Geothermal Development." Geothermal Resources Council Transactions 2 (Part 2, 1978): 457-459.

The exploitation of geothermal reservoirs is discussed on the basis of the original thermodynamic nature of the system, the estimated reserves, and the efficiency of secondary recovery. The production of primary fluids may recover only a small fraction of the reserves, and reservoir engineering techniques should be used to define and evaluate the potential of injection for enhanced recovery of heat.

Murphy, H. D. "Hydraulic-Fracture Geothermal Resérvoir Engineering." Workshop on Geothermal Reservoir Engineering. Stanford. (Stanford University Press, 1975): 174-177.

Simplified field equations are presented for the extraction of heat by circulating fluid in a hydraulic fracture in hot rock. "It is assumed that heat is conducted from the rock to a fluid circulating at a constant rate in a vertically oriented circular crack with an elliptical width profile. The equations can be solved numerically for fluid temperature and pressure with time for given crack radii and initial rock temperature. For a crack radius of $500 \mathrm{~m}$ it is shown that $25 \mathrm{MW}(+)$ can be extracted for ten years, at which time the initial rock temperature has only diminished by $5 \%$ for distances $40 \mathrm{~m}$ away from the fracture surface.

Murphy, Hugh D. and others. Preliminary Assessment of a Geothermal Energy Reservoir Formed by Hydraulic Fracturing. (Los Alamos Scientific Laboratory Report LA-UR-76-1672, Los Alamos, New Mexico, 1976)

Two 3-km-deep boreholes were drilled into hot granite in northern New Mexico to extract geothermal energy from hot dry rock. Both boreholes were hydraulically fractured to establish a flow connection. Fractureto-borehole intersection locations and in situ thermal conductivity were determined from flowing temperature logs. In situ measurements of rock permeability and compressibility showed a strong dependence on pore pressure. This was attributed to a reduction in effective stress in the intermediate principal stress direction and consequent opening of microfractures. An estimate of the minimum horizontal earth stress was derived from fracture extension pressures and found to be one-haif the overburden stress. It was found that fracture growth was remarkabiy simple to achieve in the low-permeability granite and that these fractures appear 
to be "self-propped." It is concluded that redrilling to achieve direct fracture intersection will be required before a prototype heat exchange system can be established.

Nathenson, Manuel. Physical Factors Determining the Fraction of Stored Energy Recoverable from Bydrothermal Convection Systems and Conduction-Dominated Areas. (USGS Open File Report 75-525, U.S.Geological Survey, Menlo Park, CA, 1975).

The effects of various physical reservoir properties and the thermodynamic performance of conversion equipment on the fraction of stored energy recoverable from hydrothermal convection systems and conductiondominated (hot rock) areas are discussed. Natural recharge of heat by convection and conduction as mass recharge by cold water influx is considered for water-dominated, steam-dominated, and conduction-dominated systems. It is concluded that in most cases the natural recharge potential of reservoirs is insignificant, and the heat recovery process is one of extracting stored thermal energy from a body of rock and fluids.

Various factors which affect the overall efficiency of recovery are studied using the finite stored energy concept. Boiling water or steam drive and cold water sweep processes are considered in relation to water and steam dominated hydrotherma 1 systems; forced flow through fractures is considered for extracting heat from conduction systems.

The efficiency of various power cycles as a function of the availability of the reservoir fluid is determined. Cycles considered include single and double flash steam, total flow and isobutane binary. Various design alternatives which include separation pressure, number of flash stages, and heat exchanger approach temperature are discussed. In addition, the optimum temperature conditions for process and home heating are outlined.

Papadopulos, Stavros S. "Energy Potential of Geopressured Reservoirs: Hydrogeologic Factors." First Geopressured Geothermal Energy Conference. Austin, Texas, 2-4 June 1975: 173-192.

An analysis is made to determine the possible well yields in a geopressured reservoir in Hidalgo County, Texas. A simplified reservoir model is postulated as a permeable sand layer confined above and below by shale aquitards. Production from the reservoir is analyzed using the method developed by Hantush for leaky aquifers. Pressure drop in the wellbore is also considered. Reservoir and well deliverabilities are calculated and an optimum production strategy outlined. Estimates of subsidence under this 18-well production scheme indicate that the average subsidence over the reservoir area would be about 1 meter ( 3 feet) at the end of the 20-year production period. The convertible energy delivered by the reservoir is also established. A sensitivity analysis reveals that reservoir transmissivity is the most important hydrogeological factor in the development of geopressured reservoirs. 
Ridgway, Stuart L. "Energetics of Geothermal Fluid Delivery via Two-Phase Fiow." Geothermal Resources Council Transactions 1 (1977): 225.

For geothermal well Mesa 6-1 the Hagedorn-Brown correlation and Wallis' drift flux slug flow model both predict pressure drops in reasonable agreement with experiments, but the Hagedorn-Brown method suggests increasing the well bore diameter to improve performance and Wallis' model suggests reducing it.

Sarda, Jean-Paul. Angle of Crack Propagation for a Vertical Hydraulic Fracture. (Los Alamos Scientific Laboratory Report LA-6175-MS, Los Alamos, New Mexico, 1975).

The work presented in this paper involves a model description and numerical examples for calculating fracture angle and the critical uniform borehole fluid pressure required to initiate fracture at such an angle for the present LASL Dry Hot Rock Geothermal Energy Program.

The strain-energy-density-factor (S) theory was used. The positive fracture angle $+\theta_{0}$ (the initial fracture angle of crack propagation) of a near vertical crack was predicted by using the opening and sliding node stress-intensity factors in the presence of the overburdened pressure, the last in situ horizontal principal stress, and borehole fluid pressure. The crack spread in the positive $\theta_{0}$ direction (counterclockwise) in the plane for which $S$ was minimum, $S_{\min }$. It was verified that $S_{\min } \geqslant S_{c}$. The quantity $S_{c}$ has been defined as the critical value of $S$, and remains essentially constant.

Sato, Ko. "On the Structural Characters and Simulations of Rock Fracturing of Geothermal Areas in Northeastern Japan." Second U.N. Symposizm on the Development and Use of Geothermal Resources. San Erancisco, 20-29 May 1975: 575-581.

Geothermal reservoir rocks are often hard and nonporous with the result that wells produce from fractures rather than from a porous matrix. To obtain knowledge of the rock fracturing system in the geothermal areas, computer simulations have been attempted on the basis of the finite element method. The results of the study have shown that the formation of rock fracturing is related to the condition of stress and rock properties in each area. To analyze simulations composed of two-dimensional factors, the Coulomb-Mohr criterion has been applied. It is concluded that the fractures tend to develop near the upper parts of anticlinal structures, and near the lower and upper parts of synclinal structures in the case of folding, and at the roof and hood of the intrusive body during intrusion. 
Sharma, Devrag and Maini, Tidu. "Geothermal Energy from a Borehole in Hot Dry Rock - A Preliminary Study." Workshop on Geothermaz

Reservoir Engineering. Stanford. (Stanford University Press, 1975): 258-266.

The heat transfer between circulated water and hot rock in a single concentric annulus geothermal well is studied. In a simplified initial approach, the heat gained by the water is calculated for steady flow conditions and rock temperatures. The heat transferred when fluid is pumped down the outside annulus and back up the central pipe is shown to be superior to the reverse process.

A more sophisticated approach, where cooling of the rock is considered, is also presented; and the coupled fluid-rock energy equations are solved numerically using an integrated finite difference technique. It is concluded that more rock contact area must be provided through hydraulic fracturing or explosive stimulation for effective energy yields to be obtained.

Tester, Jefferson W. GeothermaZ Energy from Dry Hot Rock Reservoirs. (Los Alamos Scientific Laboratory Report LA-UR-75-2315), LoS Alamos, New Mexico, 1976).

The concept and potential of creating and utilizing man-made geothermal reservoirs in dry hot rock is discussed. Specific references are made to the current progress of the Los Alamos Scientific Laboratory's program directed toward the extraction of geothermal heat from the hydraulically-fractured regions of hot granite underlying the Jemez Plateau of northern New Mexico. The engineering aspects of the downhole reservoir, including permeation, fluid circulation, geochemistry, heat extraction rates, thermal stress cracking, and reservoir lifetime are qualitatively discussed.

Tomasson, Jens. "Use of Injection Packer for Hydrothermal Drit Thole Stimulation in Iceland. "Second U.N. Symposium on the Development and Use of Geothermat Resources. San Francisco, 20-29 May 1975: 1821-1827.

The use of injection packers to increase the productivity of geothermal drill holes in Iceland is described. Water is pumped into producing formations which are sealed from surrounding horizons with inflatable packers. Three to four-fold increases in production have been obtained which are attributed to the opening of producing horizons clogged by drill cuttings and circulation materials and to the removal of soluble minerals near the wellbore. The injection packer has also been useful for determining the hydrologic characteristics of producing formations separated by impervious boundaries. 
Tsang, Chin Fu and Witherspoon, P. A. "An Investigation of Screening Geothermal Production Wells from Effects of Reinjection." Workshop on Geothermal Reservoir Engineering. Stanford. (Stanford University Press, 1975): 62-64.

The steady-state reservoir model of Gringarten and Sauty, which is based on single-phase potential flow in an isotropic homogeneous reservoir, is used to study the effects of screening geothermal production wells from the effects of injection. It is shown that a screening well placed between an injection and production well through which fluid is withdrawn and placed back into the injection well can significantly increase the time taken for cool injected water to break through into the production well. The breakthrough times are calculated for a range of extraction rates and well position, and it is shown that breakthrough times can be doubled with proper selection of these parameters. In addition, the thermal front propagation rate is studied with significant increases in the energy extraction being obtained when screening wells are used.

Tsang, Chin Fu and others. "A Study of Alternative Reinjection Schemes for the Cerro Prieto Geothermal Field, Baja, California, Mexico." Geothermal Resources Council Iransactions 2 (Part 2, 1978): 659-662.

Injection of brine into the Cerro Prieto geothermal reservoir is studied. A number of different injection strategies are explored which are based on the existing production well array in the reservoir. The single-phase two-dimensional steady-state flow model of Gringarten and Sauty is used to predict the "breakthrough" time when cool water reaches the production wells. It is concluded that the injection of cool brines could be safely performed without premature reduction in temperature of the produced fiutds.

Weertman, J. and others. Heat Extraction from Hot Dry Rock Masses, Progress Report, August 1, 1975 to January 31, 1976. (Northwestern University, Evanston, Illinois, 1976).

Progress on theoretical support for the Los Alamos Hot Dry Rock project is described. The growth rate of a penny-shaped crack during hydraulic fracturing has been investigated. It is verified that the fracturing fluid cannot penetrate the entire domain of a crack when the crack is moving. Model experiments have shown that joining of two hydraulic fractures may easily. lead to unsatisfactory results because the connection between the two cracks tends to be very small in size. The moment the cracks join, fluid flows into the larger crack making it grow, and further pressurization does not increase the size of the connection." Based on the differential equations for the two-dimensional water flow in a crack, a variational principle was applied in conjunction with quadrilateral fournode finite elements to obtain matrix ordinary differential equations in time for the nodal values of pressure, water flux and temperature. The results indicate the possibility of formation of closed loop eddy currents. In addition, the formation of secondary cooling cracks which could greatly increase the rates of heat withdrawal was studied by finite elements. 


\subsection{Interpretation of Production Trends}

As would be expected, data on observed trends in reservoir properties due to production has resulted from experience at The Geysers, Larderel1o, and Wairakei areas: For example, Bolton [1970] describes the changes which have taken place in underground pressures and temperatures and in the nature of the discharge due to extensive exploitation at Wairakei. Atkinson [1977] adapts oil industry-developed reservoir decline curve techniques to production data from the Serrazzano reservoir. An energy balance equation is developed and used to match past production history and predict future trends. Brigham [1977] has also adapted oil industry techniques and applies standard pressure/compressibility plots to hydrothermal systems to demonstrate the effect of a boiling phase on the behavior of these systems. A similar approach has been used by Rivera-R [1978] to describe production trends at Cerro Prieto.

Correlation of pressure-temperature trends with structural features has been useful in defining the hydrological characteristics of the reservoir and delineating areas of recharge to the system. For example, Cataldi [1970] attributes water level and temperature changes in the Travale field to recharge through aquifer type rock on the periphery of the field. Ferrara [1970] describes the decline in pressures at Caste1nuovo, Italy, in terms of a reservoir with minimal recharge and a boiling surface which is being progressively lowered due to production. Pressure transient analysis and production history are used by Lipman [1977] to assess the future potential at The Geysers. The data indicate a number of sink areas within which wells have similar responses. Boundaries permit pressure propagation between sinks.

A number of indirect techniques have been established for monitoring flow and heat transfer in producing geothermal systems. Mahon [1970] and Ellis [1964] outline chemlcal sampling and interpretive methods for describing movement of fluids, recharge areas and boiling zones in hydrothermal areas. Glover [1970] describes changes in gas composition over a ten-year production period at Wairakei in terms of boiling and cold water encroachment to the reservoir.

Nathenson [1976] outlines a method whereby recharge induced by reservoir drawdown can be determined from the nature of shallow temperature gradients in cold wells on the periphery of the field. Wooding [1974] evaluates temperature and pressure changes in deep wells drilled up to five miles from the Wairakei geothermal reservoir on the basis of production history of Wairakei and the general geology of the area.

Hunt [1970] and Isherwood [1977] correlate gravity changes at Wairakei with mass withdrawal from these reservoirs. Isherwood submits that the boiling level in vapor dominated systems can be established using this method. Hunt proposes that local anomalies in gravity can indicate the areas in which major production has occurred. 
Monitoring and interpretation of production trends will continue to be a valuable tool for defining important reservoir mechanisms which cannot be assessed directly. When incorporated into reservoir models, these effects will improve the reilability of predicted performance. The data bank that will be accumulated as a result of field monitoring during production will provide the experience from which a more general understanding of geothermal phenomena will evolve.

Atkinson, P. G. and others. "Analysis of Reservoir Pressure and Decline Curves in Serrazzano Zone-Larderello Geothermal Field." Proceedings of the Iarderello Workshop on Geothermal Resoirce Assessment and Reservoir Engineering (1977): 209-232.

This paper presents the application of production decline curve analyses to vapor-dominated geothermal fluid reservoirs. The need for incorporation of an energy balance equation to the wel1 accepted oil and gas reservoir pressure and decline curve analyses is presented. Use of the $p / Z$ curves for the vapor dominated Serrazzano Zone-Larderello geothermal reservoir indicate the noticeable effect of a curved steam-water interface on $p / Z$ behavior. Production decline curves for the same field are compared with "type curves" and future production trends are assessed. A compilation of geology, $\mathrm{p} / \mathrm{Z}$ graph and flow rate decline is used to define the exploitation history of the Serrazzano geothermal field.

Bolton, R. S. "Behavior of the Wairakei Geothermal Field During Exploitation." U.N. Symposizm on the Development and Use of Geothermal Resources. Pisa, Italy, 22 September - 1 October 1970: 1426-1439.

This paper describes the changes which have taken place in the underground pressures and temperatures and in the nature of the discharge from the Wairakei geothermal field during exploitation. The relationship between these factors is discussed, and it is concluded that the Wairakei system consists basically of a highly permeable hot water aquifer contained within almost impermeable boundaries. Under exploitation, the dominant influence on the system is the saturation temperature-pressure relationship for water, but the withdrawal of steam from the upper levels and the existence of a hot inflow at the lower levels have also played an important part.

Brigham, William E. "p/Z Behavior for Geothermal Steam Reservoirs." Society of Petrolezon Engineers Journal 17 (December 1976): 407-412.

A lumped parameter model is developed to predict the pressure and temperature versus production history of vapor-dominated reservoirs. These predictions are compared with normal hydrocarbon gas reservoirs using the standard $p / Z$ plots. The results show that the presence of a boiling water phase will have a considerable effect on the pressure 
behavior of such systems. Further, the porosity of the system will have a marked effect. Extrapolations of early data will be optimistic if the porosity is low and pessimistic if the porosity is high. In all cases, the steam zone will remain at the original temperature, though the temperature of the boiling water drops as the pressure declines.

Cataldi, R. "Contribution to the Knowledge of the Larderello Geothermal Region: Remarks on the Travale Field." U.N. Symposium on the Development and Utilization of Geothermal Resources. Pisa, Italy, 22 September-1 Oatober 1970: 587-602.

Geological, hydrogeological and physical data obtained from studies, prospects, and deep drilling carried out in the last two decades at the Travale geothermal field, Italy, are summarized. The existence of a large recharge area close to this field has brought about a rapid change in geothermal phenomena due to the drainage of producing wells. This is evidenced by a change in the physical characteristics of the fluid from superheated steam to saturated steam and/or hot water. The time scale of the evaluation can be determined on the basis of systematic measurement of hydrostatic levels and temperatures in wells.

Celati, R. and others. "Interactions Between the Steam Reservoir and Surrounding Aquifers in the Larderello Geothermal Field." Geothermics 2 (Numbers 3-4, 1973): 174-185.

Isotopic analyses of steam samples from Larderello geothermal field are studied in order to reveal the interference between the steam field and surrounding aquifers. The steam produced is made up of a mixture of waters which have undergone different circulation or hydrogeological conditions. Variations with time reveal the possibility of utilizing isotopic analyses for studying the field's evolution and the effects on the field of exploitation.

Ellis, A. J. "Geothermal Drillholes: Chemical Investigations." Proceedings of U.N. Conference on New Sources of Energy 2 (1964): 209-217.

The chemistry of spring and drillhole discharges from a geothermal system are used to infer some of the fluid and heat transport processes occurring in the system. Chemical analyses of steam and water from about 60 deep production holes at Wairakei, New Zealand, are used to derive patterns of water and steam movement. From this type of information, improved positions for future drill holes can be selected.

Ratios of ions, such as $\mathrm{Na} / \mathrm{K}, \mathrm{Na} / \mathrm{Rb}, \mathrm{Na} / \mathrm{Ca}$, are useful indicators of water movement while the composition $\left(\mathrm{CO}_{2}, \mathrm{H}_{2} \mathrm{~S}, \mathrm{NH}_{3}\right)$ and content of gas in the steam give details on conditions of steam separation from hot water. 
Ferrara, G: C. and Panichi, C. "Remarks on the Geothermal Phenomenon in an Intensively Exploited Field. Results of an Experimental Well." U.N. Symposizm on the Development and Utilization of Geothermal Resoirces. Pisa, Italy, 22 September-1 October 1970: 578-586.

The decrease of pressure and flows in the steam areas of Castelnuovo and Larderello have been caused by the progressive lowering of the evaporating surface. This phenomena occurs because of the ever-increasing drainage due to the continuous exploitation and because of the low waterfeed from the deeper layers to the steam producing layers. An attempt was made using a deep well, drilled in the most heavily worked area, to find out whether there was any possibility of tapping steam at higher pressure from depths below the present productive layers. This well reached 1097 meters and went into production at three different depths. The fluid obtained at each producing level was separately tested and different physical, chemical and isotopic characteristics were recorded.

Glover, R. B. "Interpretation of Gas Composition from the Wairakei Field Over Ten Years." U.N. Symposizm on the Development and Utilization of Geothermal Resources. Pisa, Italy, 22 September-1 October 1970: 1355-1366.

Data from surveys of the gas content of Wairakei drillhole discharges over a period of ten years were used to calculate theoretical curves relating the percentage of steam separation from the original water to the carbon dioxide content, hydrogen sulfide content, and the carbon dioxide/hydrogen sulfide ratio in both the separated steam and the residual water. From a comparison of the actual gas concentrations of drillhole discharges with the theoretical curves, it was possible to deduce the underground separation and missing processes taking place and to follow changes in downhole pressures and temperatures. Changes in $\mathrm{CO}_{2}$ and $\mathrm{H}_{2} \mathrm{~S}$ concentrations were attributed to two possible causes: nonequilibrium steam separation or multistage separation prior to discharge. In addition, the monitored change in $\mathrm{CO}_{2}$ and $\mathrm{H}_{2} \mathrm{~S}$ levels during a partial field shutdown was indicative of encroachment of $\mathrm{coOl}$ water into the aquifer.

Hunt, T. M. "Net Loss from the Wairakei Geothermal Field, New Zealand." U.N. Symposium on the Development and Utilization of Geothermal Resources. Pisa, 22 September - 1 October 1970: 487-491.

Using a gravimeter, a method of monitoring the net mass loss from a geothermal field under exploitation is described. Measurements of the value of gravity at 50 benchmarks at the Wairakei geothermal field, New Zealand, show that differences of up to $0.5 \mathrm{mgal}$ have occurred between 1961 and 1967, and up to $0.1 \mathrm{mgal}$ between 1967 and 1968. These differences, corrected for known changes in elevation, reflect the net mass of water lost from the aquifer. The net loss between 1961 and 1967 is determined as being about $2.9 \times 10^{14} \mathrm{~g}$ and hence only about $20 \%$ of the water drawn off was replaced, but between 1967 and 1968 there was little or no net loss. The gravity method can also give an indication of the area from which the water has been drawn. 
Isherwood, WHIliam F. "Reservoir Depletion at The Geysers, California." Geothermal Resources Coincil Transactions 1 (1977): 149.

Decreases in gravity measured at The Geysers between June 1974 and February 1977 indicate that water vapor being withdrawn from the field is not being replenished significantly by natural recharge. Mass balance equations based on the estimated withdrawal and the observed gravity decrease provide constraints to the recharge rate and the depth at which liquid water flashes to steam.

Lipman, S. C., Strobel, C. J., and Gulati, M. "Reservoir Performance of The Geysers Field." Proceedings of the Larderello Workshop on Geothermal Resource Assessment and Reservoir Engineering (1977): 234-255.

The production performance of The Geysers geothermal reservoir is discussed. The pressure response of the reservoir is graphically presented and used to estabilish the major drainage areas. The use of transient pressure analysis and monitoring of static water levels in shut-in wells to estabi ish reservoir boundary properties, production horizons, permeability and porosity is also outlined. It appears that the reservoir consists of a number of sinks within which propagation of pressure fluctuations is rapid; however, these are separated by boundaries which prevent pressure communication between sinks. From the available production and exploration data, it is apparent that The Geysers area will be able to support future expanston of the generation capacity in addition to the already planned 908 MWe.

Mahon, W. A. J. "Chemistry in the Exploration and Exploitation of Hydrothermal Systems." U.N. Symposizm on the Development and Utilization of Geothermal Resources. Pisa, 22 September-1 October 1970: $1310-1322$.

Experimental information on the geochemistry of individual elements in high temperature rock-water environments, and thermodynamic data for solution equilibria and gas solubilities have enabled detailed interpretations to be made of natural hot water and steam composition.

The lateral extent and chemical uniformity of the deep system can often be judged, and zones of high rock permeability connecting the aquifer at depth to the surface is outlined. Minimum estimates of the deep water temperature may be made from the concentration of constituents such as silica, magnesium and fluoride, and from the ratio of sodium to potassium in the spring waters. The tendency for deposition of minerals, particularly calcite, in the deep aquifer during exploitation can sometimes be assessed from the carbon dioxide and ion concentrations in the surface flows; information on rock types in the deep system may be obtained from the surface water chemistry.

The chemistry of fluids discharged from drillholes during exploration and production drilling provides information on the temperature of the 
water supplying the drillholes, the migration of fluids and the distribution of steam and water in the system, changes in temperatures and pressures in the aquifer during exploration, and the possibility of minerals deposition. Examples are given of interpretations of this type from the New Zealand hydrothermal areas of Wairakei, Broadlands, Waiotapu, Orakeikorako, and Ngawha.

Martin, J. C. "Fluid Flow in Geothermal Reservoirs." Workshop on Geothermal Reservoir Engineering. Stanford. (Stanford University Press, 1975): 42-44.

The pressure-temperature behavior of geothermal reservoirs produced from various initial thermodynamic conditions is discussed. Internal steam drive in hot water systems and the essentially isothermal production from steam reservoirs is qualitatively outlined.

Nathenson, Manuel. "The Effects of a Step Change in Water Flow on an Initially Linear Profile of Temperature." Second Workshop on Geothermal Reservoir Engineering. Stanford. (Stanford University Press, 1976): 40-43.

It is proposed that estimates of the surface recharge into a hydrothermal system which has drawdown under production can be made by monitoring changes in temperature distribution. The one-dimensional energy equation is written and solved analytically for the cases of flow through a semiconfining layer with a fixed upper surface temperature. It is shown that measurable transient temperature effects are produced for realistic seepage velocities and these can be used to estimate the level of local recharge.

R-Rivera, Jesus. "Application of Type Curve Procedures for the Analys is of Production Data from Geothermal Wells." Geothermal Resources Council Transactions 2 (Part 2, 1978): 575-578.

Decline curve analysis was applied to production data from 18 wells in the Cerro Prieto geothermal field. Type curve matching of the data showed that future production of different wells could be forecast on the basis of one of the three common types of decline - exponential, hyperbolic, or harmonic.

R-Rivera, Jesus. "Decline Curve Anąlysis - A Useful Reservoir Engineering T001 for Predicting the Performance of Geothermal We11s." Geothermal Resources Council Transactions 1 (1977): 257-259.

The theory of decline curve analysis is used to evaluate the production histories of several wells in the Cerro Prieto geothermal field. Fracture flow, well damage and mineral deposition are identified. It is concluded that decline curve analysis provides a useful tool for interpreting production trends in hydrothermal systems. 
Seki, Arthur S., Chen, Bill H. and Takahashi, Patrick K. "Geothermal Reservoir Engineering: Performance Matching and 'Predicting." American Society of Civil Engineers, Joumal of the Power Division 104 (1978): 169-181.

A lumped parameter computer model for production from a hydrothermal system is developed. The model is applied to interpretation of production performance in order to estimate initial reservoir conditions. The unknown initial conditions are optimized using a least square fit between predicted and measured data. The model is tested for the cases of compressed liquid, saturated liquid and dry steam reservoirs. A sensitivity analysis is performed in order to establish upper and lower constraints on each parameter. The performance matching program was found to be most sensitive to the initial mass parameters.

Tolivia, Enrique M. "Evaluation of the Geothermal Potential of Cerro Prieto, Baja, California, Mexico." Second U.N. Symposizm on the Development and Use of Geothermal Resources. San Francisco, 20-29 May 1975: 279-281.

The geothermal potential of the Cerro Prieto geothermal field is estimated on the basis of existing production data and the results of drilling on the periphery of the production area. Permeability and porosity estimates for the reservoir are based on the known stratigraphy and results from analysis and geophysical logging. The production required to sustain a 150 MWe power station is determined and the corresponding reservoir life is calculated for the case of drainage from the immediate already exploited area only, and from a larger hydraulically influenced area which is calculated from well drawdown data. In both cases the estimated life is sufficient to sustain production, and it is concluded that these conservation estimates could be significantly increased by including the effect of recharge.

Wilson, S. H. "Statistical Interpretation of Chemical Results from Drillholes as an Aid to Geothermal Prospecting and Exploitation." U.N. Symposium on the Development and Utilization of Geothermal Resources. Pisa, 22 September - 1 October 1970: 1323-1339.

Methods for monitoring the trends in chemical data from producing geothermal reservoirs are studied. It is suggested that changes in chloride, silica, $\mathrm{Na} / \mathrm{K}$ ratios, and $\mathrm{CO}_{2}$ content of production well discharges can be used to estimate average changes in downhole temperature and enthalpy. Graphical and statistical methods for determining these trends are discussed. 
Wooding, R. A. and Grant, M. A. Taupo Geothermal Bydrology. (Department of Scientific and Industrial Research, Applied Mathematics Division, Report 22, Wellington, New Zealand, 1974).

Measured pressure drops in deep bores drilled in the Tauhara-Taupo area appear to be due to drawoff from the Wairakei geothermal field, (approximately 5 miles away) and suggest a model of pressure-wave propagation through a compressible aquifer composed of saturated volcanic ash. The expected displacements in the aquifer are calculated and shown to be consistent with observed features.

Changes in the temperature of warm springs at Taupo, together with decreased flow and chloride levels, imply changes in the steam supply heating groundwater. The increase in enthalpy associated with pressure drop in the discharge of steam from a porous block is considered as a possible explanation for the observed field phenomena. 
The 1 ist of references includes all items annotated in Part II. Additional citations were included. While these references were not relevant to the four subject areas, it was the opinion of the authors, that the literature did contain valuable material in geothermal reservoir-related areas. Such references have been indicated by an asterisk (*). 


\section{REFERENCES}

Aamodt, R. Lee. Artificial Geothermal Reservoirs in Hot Volcanic Rock. (LA-UR-73-1995). LoS Alamos: Los Alamos Scientific Laboratory, [1973].

*Addoms, J. R. and Gracey, C. M. "Wellsite Verification Testing of an Advanced Geothermal Primary Heat Exchanger (APEX)." Geothermal Resources Council Transactions 2 (Part 1 1978): 1-4.

*Aktan, T. and Farouq Ali, S. M. Effect of Cyclic and In Situ Heating on the Absolute Permeabilities, Elastic Constants, and Electrical Resistivities of Rocks. Dallas: Society of Petroleum Engineers Paper No. 5633, 1975.

Aladiev, I. T. and others. "Heat and Mass Transfer Processes in Aquifer Systems with Artificially Increased Fracturing." 2nd U.N. Symposium on the Development and Use of Geothermal Resources. San Francisco, CA., U.S.A., 20-29 May, 1975: 1529-1535,

*A1-Hussainy, R. "The Flow of Real Gases Through Porous Media." A.I.M.E. Transactions 237 (1966): 624-636.

*Altseimer, John H. Technical and Cost Analysis of Rock-Melting Systems for Producing Geothermal Wells. (LA-6555-MS). Los Alamos: Los Alamos Scientific Laboratory, [1976].

Arihara, Norio. A Study of Non-Isothermal Single and Two-Phase Flow Through Consolidated Sandstones. (SGP-TR-2). Stanford: Stanford University, [1974].

*Aruna, Muhammadu, and others. Effect of Temperature and Stress on the Absolute Permeability of Sandstones and Limestones. Springfield: National Technical Information Service, [1977].

Assens, Guy E. "Derivation, by Averaging, of the Equations of Heat, Mass and Momentum Transfer in a Geothermal Reservoir." 2nd Workshop on Geothermal Reservoir Engineering. Stanford: Stanford University, December 1-3, 1976: 268-289.

Atherton, Robert, W. "The Use of General Sensitivity Theory to Analyze the Geothermal Reservoir Model's Sensitivity to the Permeability Functions." Workshop on Geothermal Reservoir Engineering. Stanford: Stanford University Press, 1975.

*Atkinson, David J. "Geothermal Development in Reno." Geothermal Resources Council Transactions 2 (Part 1 1978): 21-22.

Atkinson, P. G. and others. "Analysis of Reservoir Pressure and Decline Curves in Serrazzano Zone-Larderello Geothermal Field." Proceedings of the Larderello Workshop on Geothermal Resources Assessment and Reservoir Engineering (1977): 209-232. 
Atkinson, Paul G. Mathematical Modelling of Single-Phase Non-Isothermal. Fluid Flow Through Porous Media. Ph.D. dissertation, Stanford University, 1976.

Atkinson, P. and others. "Well-Testing in Travale-Radicondoli Field." Proceedings of the Larderello Workshop on Geothermal Resources Assessment and Reservoir Engineering (1977): 1-12.

Austin, Arthur L. and others. LLL Geothermal Energy Program Status Report January 1976 - January 1977. (Lawrence Livermore Laboratory Report UCR[-50046-76). Springfield: National Technical Information Service, [1977].

Averiev, V. V. "The Technique of Testing Geothermal Wells." Proceedings of U.N. Conference on New Sources of Energy 2 (1964): 54-59.

Baba, K. and others. "A Study of the Reservoir at the Matsukawa Geothermal Field." U.N. Symposium on the Development and Utilization of Geothermal Resources, Pisa, Sept. 22-0ct. 1, 1970: 1440-1447.

Balagna, John. Geothermal Chemistry Activities at LASL. January - December 1975. (LA-6443-PR). Los ATamos: Los ATamos Scientífíc Laboratory, [1976].

*Bannerman, J. K. "Geothermal Drilling Fluid Systems." Geothermal Resources Council, Transactions 2 (Part 1 1978): 27-29.

Banwell, C. J. "Geothermal Drillholes: Physical Investigations." Proceedings of U.N. Conference on New Sources of Energy 2 (1964): 60-71.

Barelli, A. and others. "Build-Up and Back-Pressure Tests on Italian Geothermal Wells." U.N. Symposium on the Development and Utilization of Geothermal Resources, Pisa, Sept. 22-0ct. 1, 1970: 1537-1546.

Bare11i, A. and others. "Horner Method Applied to Buildup Tests on Travale 22 We11." Workshop on Geothermal Reservoir Engineering. Stanford: Stanford University Press, 1975: 101-112.

Barelli, Antonio and others. "An Interference Test in Alfina Geothermal Fieid (Northern Latium, Italy)." Workshop on Geothermal Reservoir Engineering. Stanford: Stanford University Press, 1975: 93-100.

Barelli, A. and others. "Progress Report on a Mathematical Model of a Parallelepiped Reservoir with No Penetrating Wellbore and Mixed Boundary Conditions." Workshop on Geothermal Reservoir Engineering. Stanford: Stanford University Press, 1975: 242-246.

*Barkman, James H. "Learning Curve Concept Applied to the Development of a Hydrothermal Resource." Geothermal Resources Council, Transactions 2 (Part 1 1978): 31-33. 
*Barnes, H. L. and Rimstidt, J. P. "Control of Silica Scaling." Workshop on Geothermal Reservoir Engineering. Stanford: Stanford University Press, 1975: $185-191$.

*Batchelor, G. K. "Heat Transfer by Free Convection Across a Closed Cavity Between Vertical Boundaries at Different Temperatures." Quarterly of Applied Mathematics 62 (1954): 209-233.

*Batzle, Michael L. and Simmons, Gene. "Assessing Geothermal Areas During Drilling." Geothermal Resources Council, Transactions 2 (Part 1 1978): 13-15.

Bazant, Z. P. and Nasser, Nemat and Ohtsubo H. "Finite Element Solution of Geothermal Energy Extraction." Workshop on Geothermal Reservoir. Engi neering. Stanford: Stanford University Press, 1975: 232-239.

Bernard, William J. "Reservoir Mechanics of Geopressured Aquifers." First Geopressured Geothermal Energy Conference. Austin, Texas, 2-4 June; 1975: $157-169$.

Black, H. T. A Subsurface Study of the Mesa Geothermal Anomaly, Imperial Valley, California. Springfield: National Technical Information Service, 1975.

*Blake, Thomas R. and Garg, Sabodh K. "On the Species Transport Equation for Flow in Porous Media." Water Resources Research 12 (1976): 748-750.

*Bloomster, C. H. "Economic Modeling for Geothermal Reservoirs and Powerplants." Workshop on Geothermal Reservoir Engineering. Stanford: Stanford University Press, 1975. p. 167-168.

Bodvarsson, Gunnar. "Evaluation of Geothermal Prospects and the Objectives of Geothermal Exploration." Geoexploration 8 (1970): 7-17.

Bodvarsson, Gunnar. "Fracture Flow in Geothermal Reservoirs." Workshop on Geotherml Reservoir Engineering. Stanford: Stanford University Press, 1975: 45-49.

Bodvarsson, Gunner. "Geothermal Resource Energetics." Geothermics 3 (No. 3, 1974): 83-92.

Bodvarsson, Gunnar. "On the Temperature of Water Flowing Through Fractures." Journal of Geophysical Research 74 (1969): 1987-1992.

*Bodvarsson, Gunnar. "Physical Characteristics of Natural Heat Resources in Iceland." Proceedings of U.N. Conference on New Sources of Energy 2 (1964): 82-89.

Bodvarsson, Gunnar. A Secondary Recovery Method for the Extraction of Geothermal Energy. Corvallis: Oregon State University, 1977.

Bodvarsson, Gunnar. "Thermoelastic Phenomena in Geothermal Systems." 2nd U.N. Symposium on the Development and Use of Geothermal Resources. San Francisco, CA. , U.S.A., 20-29 May, 1975: 903-907. 
Bolton, R. S. "Behavior of the Wairakei Geothermal Field During Exploitation." U.N. Symposium on the Development and Utilization of Geothermal Resources. Pisa, Sept. 22-0ct. 1, 1970: 1426-1439.

Bolton, R. S. "Management of a Geothermal Field." UNESCO Earth Sciences 12 (1973): 175-184.

Bories, Serge. "Fundamental Study of Changing of Phase in Porous Materials." Workshop on Geothermal Reservoir Engineering. Stanford: Stanford University Press, 1975: 247-248.

Bories, S. A. and Combarnous, M. A. "Natural Convection in a Sloping Porous Layer." Journal of Fluid Mechanics 57 (1973): 63-79.

*Botset, H. G. "Effect of Pressure Reduction upon Core Saturation." A.I.M.E. Transactions 132 (1939): 172-183.

*Bourgoyne, A. T. and others. Shale Water as a Pressure Support Mechanism in Superpressure Reservoirs. Dallas: Society of Petroleum Engineers Paper No. 3851 .

*Brace, W. F. "Resistivity of Saturated Crustal Rocks to $40 \mathrm{~km}$ based on Laboratory Measurements." In The Structure and Physical Properties of the Earth's Crust, pp. 243-255. Washington D.C.: American Geophysical Union, 1971.

Bredehoeft, J. D. and Papadopulos, I. S. "Rates of Vertical Groundwater Movement Estimated from the Earth's Thermal Profile." Water Resources Research 1 (1965): 325-238.

Brigham, William E. "Heat and Fluid Flow Experiments to Measure Geothermal Reservoir Physical Parameters." Workshop on Geothermal Reservoir Engineering. Stanford: Stanford University Press, 1975: 26-36.

Brigham, Willian $E_{\text {. }}$ and Morrow, B. William. "p/Z Behavior for Geothermal Steam Reservoirs." Society of Petroleum Engineers Journal 17 (December 1976): 407-412.

*Brooks, Royal H. and Corey, Arthur T. "Properties of Porous Media Affecting Fluid Flow." ASCE Journal of Irrigation and Drainage Division IR2 (1966): 61-88.

*Brown, R. F. and Signor, D. C. "Artificial Recharge--State of the Art." Underground Waste Management and Artificial Recharge 2 (1973): 668-686.

Browne11, D. N., Garg, S. K. and Pritchett, J. W. Computer Simulation of Geothermal Reservoirs. Dallas: Society of Petroleum Engineers Paper No. $5381,1975$.

*Buckley, S. E. and Leverett, M. C. "Mechanism of Fluid Displacement in Sands." A.I.M.E. Transactions 146 (1942): 107-116. 
Budd, Chester F. Jr. "Steam Production at the Geysers Geothermal Field." In Geothermal Energy: Resources, Production, Stimulation, Chapter 6. Edited by Paul Kruger and Carel Otte. Stanford: Stanford University Press, 1973.

*Burrows, W. "Geothermal Energy Resources for. Heating and Associated Application in Rotorua and Surrounding Areas." U.N. Symposium on the Development and Utilization of Geothermal Resources. Pisa, Sept. 22-0ct. 1, 1970: 1662-1669.

*Busse, F. H. "On Howard's Upper Bound for Heat Transport by Turbulent Convection." Journal of Fluid Mechanics 37 (1969): 457-477.

Cady, C. V., Bilhartz, H. L., and Ramey, Henry J. Jr. "Model Studies of Geothermal Stream Production." Water--1972 AIChE Symposium Series. New York: American Institute of Chemical Engineers, 1972: 445-452.

Cady, Gilbert. Model Studies of Geothermal Fluid Products. Ph.D. dissertation, Stanford University, 1969.

Calamai, A. and others. "A Reinjection Experiment in Vico 1 Well." Geothermics 2 (Numbers 3-4, 1973): 117-118.

*Calhoun, John C. Jr., Lewis, Maurice Jr, and Newman, R. C. "Experiments on the Capillary Properties of Porous Solids." A.I.M.E. Transactions 186 (1949): 189-196.

Campbe11, Thomas A. "Analysis of Geothermal Well Pumping." Geothermal Resource Counci1, Transactions 2 (Part 1, 1978): 63-66.

*Cardwell, W. T. Jr. and Parsons, R. L. "Average Permeabilities of Heterogeneous 0 il Sands." A.I.M.E. Transactions 160 (1945): 34-42.

*Case, Charles W. and Wilde, Pat. Methods for Geothermal Reservoir Detection Emphasizing Submerged Environments. (LBL 4495). Berkeley: Lawrence Berke1ey Laboratory, [1976].

*Casse, Francis Jose. Effect of Temperature and Confining Pressure on Fluid Flow Properties of Consolidated Rocks. Ph.D. dissertation, Stanford University. 1975.

*Cassou, Anne-Marie., Connan, Jacques and Porthault, Bernard. "Relations Between Maturation of Organic Matter and Geothermal Effect as Exemplified in Canadian Est Coast Offshore Wells." Bulletin of Canadian Petroleum Geology 25 (1977): 174-194.

Cataldi, R. and others. "Contribution to the Knowledge of the Larderello Geothermal Region: Remarks on the Travale Field." U.N. Symposium on the Development and Utilization of Geothermal Resources. Pisa, Sept. 22Dct. 1, 1970: 587-602. 
Celati, Romano and others. "Analysis of Water Levels and Reservoir Pressure Measurements in Geothermal Wells." 2nd U.N. Symposium on the Development and Use of Geothermal Resources. San Francisco, CA., U.S.A., 20-29 May, 1975: 1583-1590.

*Celati, R. and others. "An Attempt to Correlate Kh Distribution with Geological Structure of Larderel10 Geothermal Field." Workshop on Geothermal Reservoir Engineering. Stanford: Stanford University Press, 1975: $37-41$.

Celati, R. and others. "Interactions between the Steam Reservoir and Surrounding Aquifers in the Lardere110 Geothermal Field." Geothermics 2 (Numbers $3-4 ;$ 1973): 174-185.

Celati, R. and others. "Study of Effect of Reinjection with a Mathematical Mode 1. " Proceedings of the Lardere110 Workshop on Geothermal Resource Assessment and Reservoir Engineering (1977): 308-326.

*Chan, B. K. C., Irvey, C. M. and Barry, J. M. "Natural Convection in Enclosed Porous Media with Rectangular Boundaries." Journal of Heat Transfer 92 (1970): : 21-27.

*Chappe11, Robert N. and others. "Multi-Purpose Geothermal Test and Experimental Activities at Raft River, Idaho." Geothermal Resources Council, Transactions 2 (Part 1, 1978): 83-85.

Chasteen, A. J. "Geothermal Steam Condensate Reinjection." Conference on Research for the Development of Geothermal Energy Resources, September 23-25, 1974: 340-344.

*Chatas, Angelos T. "A Practical Treatment of Nonsteady-State Flow Problems in Reservoir Systems." Petroleum Engineer 24 (In 3 Parts).

Chen, Bill. Geothermal Reservoir and Well Test Analysis: A Literature Survey. Report Prepared for National Science Foundation Research Applied to National Needs by University of Hawaii, Hilo College, 1974.

Chen, Bill H. and others. "Well Test Results from HGP-A." Geothermal Resources Council Transactions 2 (Part 1, 1978): 99-102.

Chen, Che Jen. "Man-Made Geothermal Energy Source." Nuclear Instruments and Methods 150 (1978): $365-366$.

Chen, H. K., Counsil, J. R. and Ramey, H. J., Jr. "Experimental Steam-Water Relative Permeability Curves." Geothermal Resources Council Transactions 2 (Part 1, 1978): 103-104.

Chen, Hsiu-Kuo. Measurement of Water Content in Porous Media Under Geothermal Fluid Flow Conditions. Ph.D. dissertation, Stanford University, 1977.

Chen, Jay C., Conel, James, E. "Finite-Element Solutions for Geothermal Systems." Journal of Energy 1 (1977): 364-369. 
Cheng, Ping and Lau, K. H. "The Effect of Steady Withdrawl of Fluid in Confined Geothermal Reservoirs." 2nd U.N. Symposium on the Development and Use of Geothermal Resources. San Francisco, CA., U.S.A., 20-29 May, 1975: 1591-1598.

Cheng, Ping. "Heat and Mass Transfer in Liquid Dominated Geothermal Reservoirs." Letters in Heat and Mass Transfer 3 (1976): 81-88.

Cheng, Ping. "Numerical and Analytical Studies on Heat and Mass Transfer in Volcanic Island Geothermal Reservoirs." Workshop on Geothermal Reservoir Engineering. Stanford: Stanford University Press, 1975: 219-224.

Cheng, Ping and Lau, K. H. "Numerical Modeling of Hawai ian Geothermal Resources." Geothermics 2 (Numbers 3-4, 1973): 90-93.

Cheng, Ping and Teckchandani, Lall. Numerical Solution for Transient Heating and Withdrawal of Fluid in a Liquid-Dominated Geothermal Reservoir. Honolulu: University of Hawaii, 1976.

Cheng, Ping and Lau, K. H. "Numerical Solutions for Steady Free Convection in Island Geothermal Reservoirs." Future Energy Production Systems: Heat and Mass Transfer Processes II (1976): 429-448.

Cheng, Ping and Lau, K. H. "Steady-State Free Convection in an Unconfined Geothermal Reservoir." Journal of Geophysical Research 79 (1974): 44254431.

Chicoine, S. D. and Ramey, Henry J. Vapor Pressure Lowering in Two-Phase Geothermal Systems. Dallas: Society of Petroleum Engineers Paper No. 6767, 1977.

Coats, K. H. Geothermal Reservoir Modelling. Dallas: Society of Petroleum Engineers Paper No. 6892, 1977.

Coats, K. H. and others. "Three-Dimensional Simulation of Steamflooding." A.I.M.E. Transactions 257 (1974): 573-592.

Combarnous, Michel A. and Bia, Paul. "Combined Free and Forced Convection in porous Media." Society of Petroleum Engineers Journal 11 (1971): 399-405.

Combarnous, Michel A. and Bories, S. A. "Hydrothermal Convection in Saturated Porous Media." Advances in Hydroscience 10 (1975): 231-307.

*Cooke, W. L. "Some Methods of Dealing with Low Enthalpy Water in the Rotorua Area of New Zealand." U.N. Symposium on the Development and Utilization of Geothermal Resources. Pisa, Sept. 22-0ct. 1, 1970: 2 (Part 2, 1970): 1670-1675.

*Danesh, A. and Ramey, H. J., Jr. "The Effect of Temperature Level on Absolute Permeability of Unconsolidated Silica and Stainless Stee1." Geothermal Resources Council Transactions 2 (Part 1, 1978): 137-139. 
*Davis, Stephen H. "Finite Amplitude Instability of Time-Dependent Flows." Journal of Fluid Mechanics 45 (1970): 33-48.

*Deardorff, J. W. and Willis, G. E. "The Effect of Two-Dimensionality on the Suppression of Thermal Turbulence." Journal of Fluid Mechanics 23 (1965): 337-353.

*Deardorff, J. W. and Willis,G. E. "Investigation of Turbulent Thermal Convection Between Horizontal Plates.", Journal of Fluid Mechanics 28 (1967): 675-704.

Delisle, George. Determination of Permeability of Granitic Rocks in GT-2 From Hydraulic Fracturing Data. (LA-6169-MS). Los Alamos: Los Alamos Scientific Laboratory, [1975].

*Demarest, Harold H. Jr. Application of Stress Corrosion to Geothermal Reservoirs. (LA-6148-MS). LoS Alamos: Los ATamos Scientific Laboratory [1975].

*Dennis, Bert R. and Hugh, Murphy, D. "Borehole Temperature Survey Analysis Hot Dry Rock Geothermal Reservoir." Geothermal Resources Council Transactions 2 (Part 1, 1978): 149-152.

*de Swaan 0. A. "Analytic Solutions for Determining Naturaliy Fractured Reservoir Properties for We11 Testing." Society of Petroleum Engineers Journal 16 (1976): 117-122.

Diadkin, Y. D. and Pariisky, Y.M. "Theoretical and Experimental Grounds for Utilization of Dry Rock Geothermal Resources in the Mining Industry." 2nd U.N. Symposium on the Development and Use of Geothermal Resources, San Francisco, CA., U.S.A., 20-29 May, 1975: 1609-1618.

Dodson, C. R., Goodwill D. and Mayer, E. H. Application of Laboratory PVT Data to Reservoir Engineering Problems." A.I.M.E. Transactions 198 (1953): 287-298.

Donaldson, I. G. and M. G. Grant. "An Estimate of the Resource Potential of New Zealand Geothermal Fields for Power Generation." Proceedings of the Lardere110 Workshop on Geothermal Resource Assessment and Reservoir Engineering (1977): 413-427.

Donaldson, I. G. "Estimation of Subsurface Flows and Permeabilities from Temperature and Pressure Data". U.N. Symposium on the Development and Utilization of Geothermal Resources. Pisa, Sept. 22-0ct. 1, 1970: 677683.

Donaldson, I. G. "The Flow of Stream Water Mixtures Through Permeable Beds: A Simple Simulation of Natural Undisturbed Hydrothermal Region." New Zealand Journal of Science 11 (1968): 3-23.

Donaldson, I. G. "Simulation of Geothermal Systems with a Simple Convection Mode1." U.N. Symposium on the Development and Utilization of Geothermal Resources. Pisa, Sept. 22-0ct. 1, 1970: 649-654. 
Donaldson, I. G. "Temperature Gradients in the Upper Layers of the Earth's Crust Due to Convective Water Flows." Journal of Geophysical Research 67 (1962): 3449-3459.

*Downs, W. F., Barnes, H. L. and Rimstidt, J. D. "Field Scaling Tests on Geothermal Brines." Geothermal Resources Council Transactions 2 (Part 1, 1978): $165-166$.

*Dudarev, A. N. "Endoclave, a New Device for the Study of Heat and Mass Transfer by Simulation of Geologic Bodies and Processes Under Dynamic Conditions." U.N. Symposium on the Development and Utilization of Geothermal Resources. Pisa, Sept. 22-0ct. 1, 1970: 1275-1279.

*Dundurs, J. "Model Experiments in Hydraulic Fracture." Workshop on Geothermal Reservoir Engineering. Stanford: Stanford University Press, 1975: 178-179.

Einarsson, Sveinn S., Vides, Alberto R., and Cuellar, Gustavo. "Disposal of Geothermal Waste Water by Reinjection." 2nd U.N. Symposium on the Development and Use of Geothermal Resources. San Francisco, CA., U.S.A., 20-29 May, 1975: 1349-1362.

Elder, J. W. "Numerical Experiments with Free Convection in a Vertical Slot." Journal of Fluid Mechanics 24 (1960): 823-843.

Elder, J. W. "Steady Free Convection in a Porous Medium Heated from Below." Journal of Fluid Mechanics 27 (1967): 29-48.

Elder, J. W. "Transient Convection in a Porous Medium." Journal of Fluid Mechanics 27 (1967): 609-623.

*Elder, J. W. "The Unstable Thermal. Interface." Journal of Fluid Mechanics 32 (1968): 69-96.

*Elders, W. A. "Petrology as a Practical Tool in Geothermal Studies." Geothermal Resources Council Transactions 1 (1977): 85-87.

Ellis, A. J. "Geothermal Drillholes: Chemical Investigations." Proceedings of U.N. Conference on New Sourcesof Energy 2 (1964): 208-217.

*Ellis, A. J. "Quantitative Interpretation of Chemical Characteristics of Hydrothermal Systems." U.N. Symposium on the Development and Utilization of Geothermal Resources. Pisa, Sept. 22-0ct. 1, 1970: 516-528.

Facca, G. and Tonani, F. "Natural Steam Geology and Geochemistry." Proceedings of U.N. Conference on New Sources of Energy 2 (1964): 219-228.

Faust, Charles R. and Mercer, James W. An Analysis of Finite-Difference and Finite-Element Techniques for Geothermal Reservoir Simulation. Dallas: Society of Petroleum Engineers Paper No. 5742, 1976.

Faust, Charles R. and Mercer, James W. "Mathematical Modeling of Geothermal Systems." 2nd U.N. Symposium on the Development and Use of Geothermal Resources. San Francisco, CA., U.S.A., 20-29 May, 1975: 1635-1641. 
Faust, Charles R. "Numerical Simulation of Fluid Flow and Energy Transport in Liquid-and Vapor-Dominated Hydrothermal Systems. Ph.0. dissertation, Pennsylvania State University, 1976.

Faust, Charles R. "Summary of our Research in Geothermal Reservoir Simulation." Workshop on Geothermal Reservoir Engineering. Stanford: Stanford University Press, 1975: 198.

*Fehlberg, E. L. "Shell's Activity in the Geysers Area." Workshop on Geothermal Reservoir Engineering. Stanford: Stanford University Press, 1975: 84-88.

Ferrara, G. C., Panichi, C. R. and Stefani, G. "Remarks on Geothermal Phenomenon in all Intensively Exploited Field. Results of an Experimental Well." U.N. Symposium on the Development and Utilization of Geothermal Resources. Pisa, Sept. 22-0ct. 1, 1970: 578-586.

Fisher, Leonard A., Black, Allan, R. and Fogleman, Sam. F. "Total Energy Recovery System for Agribusiness: Lake County Study." Geothermal Resources Council Transactions 2 (Part 1, 1978): 193-194.

*Foster, Theodore $D$. "Onset of Convection in a Layer of Fluid Cooled from Above." Physics of Fluids 8 (1965):-177-1774.

*Foster, Theodore D. "Stability of a Homogeneous Fluid Cooled Uniformly from Above." Physics of Fluids 8 (1965): 1249-1257.

*Fromm, Jacob E. "Numerical Solutions of the Nonlinear Equations for a Heated Fluid Layer," Physics of Fluids 8 (1965): 1757-1769.

*Frye, George. "Water Entry Below Steam Production: A Case History at the Geysers." Workshop on Geothermal Reservoir Engineering. Stanford: Stanford University Press, 1975: 39-92.

*Furumoto, Augustine S. "The Relationship of a Geothermal Reservoir to the Geological Structure of the East Rift of Kilauea Volcano, Hawaii." Geothermal Resources Council Transactions 2 (Part 1, 1978): 199-201.

*Garg, S. K. "Land Surface Subsidence Associated with Geothermal Energy Production." Workshop on Geothermal Reservoir Engineering. Stanford: Stanford University Press, 1975: 65-68.

Garg, S. K. "Pressure Transient Analys is for Two-Phase Geothermal Reservoirs." Geothermal Resources Council Transactions 2 (Part 1, 1978): 203-206.

Garg, S. K. and others, Simulation of Fluid-Rock Interactions in a Geothermal Basin. (SSS-R-74-2431). La Jolla: Systems, Science and Software, [1974].

Garg, S. K. "Two-Phase Flow in Geopressured Geothermal Wells." Energy Conversion 18 (1978): 45-51. 
Glover, R. B. "Interpretation of Gas Composition from the Wairakei Field over Ten Years." U.N. Symposium on the Development and Utilization of Geothermal Resources. Pisa, Sept. 22-0ct. 1, 1970: 1355-1366.

*Goldstein, Marvin E. and Siegel, Robert. "Analysis of Heat Transfer for Compressible Flow in Two-Dimensional Porous Media." International Journal of Heat and Mass Transfer 14 (1971): 1677-1689.

*Goss, R. D. Empirical Relationships Between Thermal Conductivity and Other Physical Parameters in Rocks. Ph.D. dissertation, University of California, Riverside; 1974.

Gould, Thomas L. "East Mesa Reservoir." Workshop on Geothermal Reservoir Engineering. Stanford: Stanford University Press, 1975: 146-152.

Gould, Thomas L. "Vertical Two-Phase Steam-Water Flow in Geothermal Wells." Journal of Petroleum Technology 26 (1974): 833-842.

Grant, M. A. Asymptotic Solution for the One-Phase Field Model and Lifetime Estimate. Technical Report No. 47). Wellington: New Zealand. Department of Scientific and Industrial Research. Applied Mathematics Division, [1976].

Grant, M. A. Broadlands - A Gas-Dominated Geothermal Field. (D.S.I.R. Report No. 46). Wellington: New Zealand. Department of Scientific and Industrial Research. Applied Mathematics Division, [1976].

Grant, M. A. Discharge of a Porous Block. (Technical Report No. 21), Wellington: New Zealand. Department of Scientific and Industrial Research. Applied Mathematics Division, [no date].

Grant, M. A. Exploitation of a Limited Two-Phase Reservoir. (Technical Report No. 41). Wellington: New Zealand. Department of Scientific and Industrial Research. Applied Mathematics Division, [1976].

*Gray, William G, and $0^{\prime}$ Neil, Kevin, "On the General Equations for Flow in Porous Media and their Reduction to Darcy's Law." Water Resources Research 12 (1976): 148-154.

*Grijalva, Ramon L. "The Fossile Fuel/Geothermal Hybrid Cycle Applied to Marginally Useful Liquid Dominated Hydrothermal Resources." Geothermal Resources Council Transactions 2 (Part 1, 1978): 227-231.

*Grindley, G. W. "Subsurface Structures and Relation to Steam Production in the Broadlands Geothermal Field, New Zealand." U.N. Symposium on the Development and Utilization of Geothermal Resources. Pisa, Sept. 22oct. 1, 1970: 248-261.

Gringarten, A. C. and Sauty, J. P. "The Effect of Reinjection on the Temperature of a Geothermal Reservoir Used for Urban Heating." 2nd U.N. Symposium on the Development and Use of Geothermal Resources. San Francisco, CA. , U.S.A., 20-29 May, 1975: 1370-1374. 
Gringarten, A. C. and Witherspoon, P. A. "Extraction of Heat from MultipleFractured Dry Hot Rock." Geothermics 2 (Numbers 3-4, 1973): 119-122.

*Gringarten, A. C. "Study of a Geothermal Field in the ASAL Active Volcanic Rift Zone (French Territory of Affars and Issas, East Africa)." Workshop on Geothermal Reservoir Engineering. Stanford: Stanford University Press, 1975: 113-116.

Gringarten, A. C. and Sauty, J. P. "A Theoretical Study of Heat Extraction from Aquifers with Uniform Regional Flow." Journal of Geophysical Research 80 (1975): 4956-4962.

Gulati, M. S. "Pressure and Temperature Buildup in Geothermal Wells." Workshop on Geothermal Reservoir Engineering. Stanford: Stanford University Press, 1975: 69-73.

*Hall, Frederick F. "Solar, Geothermal, Hydrogen, and Hydraulic Power." American Society of Civil Engineers. Journal of the Power Division 104 (1978): 71-82.

Hanson, Jonathan M. and Kasameyer, Paul W. "Prediciting Production Temperature Using Tracer Methods." Geothermal Resources Council Transactions 2 (Part 1, 1978): 257-258.

*Harban, D. C. "Geothermal Well Testing at Roosevelt KGRA, Beaver County, Utah." Workshop on Geothermal Reservoir Engineering. Stanford: Stanford University Press, 1975: 77-83.

Hardee, Harry C. and Larson, David, W. Heat Transfer in Magma Bodies. (SAND 76-0011). Springfield: National Technical Information Services, [1976].

*Hedstrom, W. E., Corey, A. T. and Duke, H. R. Models for Subsurface Drainage. (Colorado State University Hydrology Paper No. 48), Fort Collins: Colorado State University, [1971].

*Hickel, Walter J. Geothermal Energy: A National Proposal for Geothermal Resources Research. Anchorage: University of Alaska, 1972.

Hinrichs, Thomas C. "Niland Reservoir Monitoring and Evaluation Operating Program." Workshop on Geothermal Reservoir Engineering. Stanford: Stanford University Press, 1975: 143-145.

*Hoagland, James R." "Applications of Solute Equilibrium Models to the Study of Geothermal Reservoirs." Geothermal Resources Council Transactions 1 (1977): 143-145.

*Hochstein, Manfred Paul. "Geophysical Exploration of the Kawah Kamojang Geothermal Field, West Java." 2nd U.N. Symposium on the Development and Use of Geothermal Resources. San Francisco, CA., U.S.A., 20-29 May, 1975: 1049-1058. 
Holst, P. H. and Aziz, K. "Theoretical and Experimental Study of Natural Convection in a Confined Porous Medium." Canadian Journal of Chemical Engineering 50 (1972): 232-241.

Holst, P. H. and Aziz, K. "Transient. Three-Dimensional Natural Convection in Confined Porous Media." International Journal of Heat and Mass Transfer 15 (1972): 73-90.

Horne, R. N. and O'Sullivan, Michael, J. Numerical Modelling of the Wairakei Geothermal Reservoir. Dallas: Society of Petroleum Engineers Paper No. 6536, 1977.

Horne, R. N. and O'Sullivan, M. J. "Oscillatory Convection in a Porous Medium Heated from Below." Journal of Fluid Mechanics 66 (part 2, 1974): 339-352.

Horne, R. N. and Ramey, Henry, J. Jr. "Steam/Water Relative Permeabilities from Production Data." Geothermal Resources Council Transactions 2 Part 1, 1978): 291-293.

Horton, C. W. and Rogers, R. T. "Convection Currents in a Porous Medium." Journal of Applied Physics 16 (1945): 376-374.

Hsu, Y. C. and others. "Engineering Methods for Prediciting Productivity and Longevity of Hot-Dry-Rock Geothermal Reservoir in the Presence of Thermal Cracks." Geothermal Resources Council Transactions 2 (Part 1, 1978): 297-298.

Hsu, Y. C. Forced Oscillations of the Los Alamos Scientific Laboratory's Dry Hot Rock Geothermal Reservoir. (LA-6170-MS). Los Alamos: Los Alamos Scientific Laboratory.

*Hubbert, M. King. "Theory of Scale Models as Applied to the Study of Geologic Structures." Bulletin of the Geological Society of America 48 (1937): $1459-1520$.

Hunsbedt, A. and others. "Energy Extraction Experiments in the SGP Reservoir Mode 1." Stanford Geothermal Reservoir Engineering Workishop, 3rd. Stanford: Stanford University Press, 1977.

Hunsbedt, Anstein and others. "Energy Extraction Experiments in the SGP Reservoir Mode1." Geothermal Resources Council Transactions 2 (Part 1, 1978): 299-301.

Hunsbedt, Anstein and London, Alexander. "Laboratory Studies of Stimulated Geothermal Reservoirs." 2nd U.N. Symposium on the Development and Use of Geothermal Resources. San Francisco, CA., U.S.A., 20-29 May, 1975: 1663-1671.

Hunsbedt, Anstein. Laboratory Studies of Stimulated Geothermal Reservoirs. Ph.D. dissertation, Stanford University, 1976. 
Hunsbedt, A., Kruger, P. and London, A. L. "Recovery of Energy from Fractured-Stimulated Geothermal Reservoirs," Journal of Petroleum Technology 29 (1977): 940-946.

Hunsbedt, Anstein, Kruger, P. and London, A. L. Recovery of Energy from Fractured Geothermal Reservoirs. Dallas: Society of Petroleum Engineers Paper No. 5875, 1976.

Hunt, T.M. "Net Loss from the Wairakei Geothermal Field, New Zealand." U.N. Symposium on the Development and Utilization of Geothermal Resources, Pisa, Sept. 22-0ct. 1, 1970: 487-491.

Isherwood, William F. "Reservoir Depletion at the Geysers, California." Geothermal Resources Council Transactions 1 (1977): 149.

Isita, J. "Low Permeability of a Thick Geothermal Aquifer in a Highly Fractured Area as Necessary Requisite for High Steam Production." U.N. Sympsoium on the Development and Utilization of Geothermal Resources. Pisa, Sept. 22-0ct. 1, 1970: 684-697.

Isokrari, Ombo F. Natural Gas Production from Geothermal Geopressured Aquifers. Dallas: Society of Petroleum Engineers Paper No. 6037, 1976.

Isokrari, Ombo F. Numerical Simulation of United States Gulf Coast Geothermal Geopressured Reservoirs. Ph.D. dissertation, University of Texas, 1976.

*Jackson, Donald D. "Chemical Modeling of Geothermal Systems." Geothermal Resources Council Transactions 1 (1977): 151-152.

* Jacobson, W. 0. "Operational History of the Geothermal Facility at the Salton Sea KGRA." Geothermal Resources Council Transactions 2 (Part 1, 1978): $325-326$.

James R. "Factors Controlling Borehole Performance:" U.N. Symposium on the Development and Utilization of Geothermal Resources. Pisa, Sept. 220ct. 1, 1970: 1502-1515.

James, Russell. "Drawdown Test Results Differentiate Between Crack Flor and Porous Bed Permeability." U.N. Symposium on the Development and Use of Geothermal Resources. San Francisco, May 20-29, 1975: 1693-1695.

James, Russel1. Power-Life of a Hydrothermal System. 2nd Australiasian Conference in Hydraulics and Fluid Mechanics. (1965): 211B-233B.

James, Russe11. "Wairakei and Larderel10: Geothermal Power Systesms Compared." New Zealand Journal of Science 11 (1968): 708-719.

*Jin, Doo Jung. "True-Temperature Determination of Geothermal Reservoirs." Geoexploration 15 (1977): 1-9.

*Jiracek, George R. and Gerety, Michael T. "Comparison of Surface and Down Hole Resistivity Mapping in Geothermal Reservoirs in New Mexico." Geothermal Resources Council Transactions 2 (Part 1, 1978): 335-336. 
Juprasert, Sirisak, and Sayual Subir K. "A Numerical Simulator for Flow in Geothermal Wellbores." Geothermal Resources Council Transactions 1 (1977): 159-161.

Karlsson, Thorbjorn. "Casing Design for High Temperature Geothermal Wells." Geothermal Resources Council Transactions 2 (Part 1, 1978): 355-358.

Kasameyer, Paul, Thorson, Lewis, and McKee, Chester. "Modeling Thermal and Flow Fronts for Arbitrary Well Arrays." Geothermal Resources Council Transactions 1 (1977): 163-165.

Kasameyer, P. W. and Schroeder, R. "Thermal Depletion of Liquid-Dominated Geothermal Reservoirs with Fracture and Pore Permeability." Workshop on Geothermal Reservoir Engineering. Stanford: Standord University Press, 1975: $249-257$.

Kassoy, D. R. "Heat and Mass Transfer in Models of Undeveloped Geothermal Fields." 2nd U.N. Symposium on the Development and Use of Geothermal Resources, San Francisco, CA., U.S.A., 20-29 May, 1975: 1707-1711.

Kassoy, D. R. "Summary Description of Research Activities." Workshop on Geothermal Reservoir Engineering. Stanford: Stanford University Press, 1975: 23-25.

Kassoy, D. R. Transport of Mass and Energy In Porous Media Due to Natural Convection: The Geothermal Basin Problem. Springfield: National Technical Information Service, 1976.

Kassoy, D. R., Zebib, A. "Variable Viscosity Effects on the Onset of Convection in Porous Media." Physics of Fluids 18 (1975): 1649-1651.

*Keller, Joseph B. "Periodic Oscillations in a Model of Thermal Convection." Journal of Fluid Mechanics 26 (1966): 599-606.

*Kintzinger, P. R. Seismic Signal Location Program for the Los Alamos Scientific Laboratory's Dry Hot Rock Geothermal Project. (LA-6274-MS). Los Alamos: Los Alamos Scientific Laboratory, [1976].

*Klein, Frederick William. Tidal Triggering of Earth Swarms and the Reykjanes Peninsula, Iceland Earthquake Swarm of September 1972 and Its Tectonic and Geothermal Implications. Ph.D. dissertation, Columbia University, 1976.

Knapp, R. M. and others. An Analysis of Production from Geopressured Geothermal Aquifers. Dallas: Society of Petroleum Engineers Paper No. 6825, 1977.

Knapp, R. M. and Isokari, O. F. "Aspects of Numerical Simulation of Future Performance of Geopressured Geothermal Reservoirs." 2nd Geopressured Geothermal Energy Conference. University of Texas, Austin; 23-25 February, 1976: 102-161.

*Knapp, R. M., Dorfman, M. H. and Isokrari, O. F. "Geopressured Geothermal Reservoir Engineering Reserach at the University of Texas." Workshop on Geothermal Reservoir Engineering. Stanford: . Stanford University Press, 1975: $130-134$. 
Kononenko, G. N. and Tsyrul' Nikou, A. S. "Coolant Filtration in Man-Made Underground Circulation Systems." Journal of Fluid Mechanics 3 (1974): 95-98.

*Krishnamurti, Ruby. "On the Transition to Turbulent Convection Part 2. The Transition to Time-Dependent Flow." Journal of Fluid Mechanics 42 (1970): 309-320.

*Kroopnick, Peter M. and others. "Geochemistry of a Hawaii Geothermal We11-HGP-A." Geothermal Resources Council Transactions 2 (Part 2, 1978): 375-377.

Kruger, Paul. "Physical Mode1s of Stimulated Geothermal Reservoirs." Workshop on Geothermal Reservoir Engineering. Stanford: Stanford University Press, 1975: 168-173.

Kruger, Paul. "Stimulation and Reservoir Engineering of Geothermal Resources." Stanford Geothermal Program, TR-1. Stanford: Stanforf University Press, 1974.

*Kruger, Paul, and Ramey, Henry J. Jr. "Stimulation and Reservoir Engineering of Geothermal Resources." Stanford Geothermal Program, TR-28. Stanford: Stanford University Press, 1978.

Kubota, Katsundo and Aosaki, Kowaski. "Reinjection of Geochemical Hot Water at the Otake Geothermal Field." 2nd U.N. Symposium on the Development and Use of Geothermal Resources, San Francisco, CA., U.S.A., 20-29 May, 1975: 1379-1383.

Kunze, J. F., Miller, Lowell G. and Stoker, Roger C. "The Experience of Getting Water Out of the Raft River Geothermal We11s." 17th International Rock Mechanics Symposium Snowbird, Utah, August 16-17, 1976: 3B4-1-3B4-5.

Kunze, Jay F., Miller, L. G. and Stocker, R. C. "Raft River Geothermal Reservoir Engineering and Well Stimulation." Workshop on Geothermal Reservoir Engineering. Stanford: Stanford University Press, 1975: 117-123.

Kuo, Ming-Ching T., Kruger, Paul and Brigham, W. E. "Heat and Mass Transfer in Porous Rock Fragments." Stanford Geothermal Program TR-10. Stanford: Stanford University Press, 1975.

Kuwada, J. T. "Geothermal Reservoir Pressure Requirements for Production." Workshop on Geothermal Reservoir Engineering. Stanford: Stanford University Press, 1975: 157-160.

*Lantz, R. B. "Quantitative Evaluation of Numerical Diffusion (Truncation Error)." A.I.M.E. Transactions 251 (1971): 315-320.

Lapwood, E. R. "Convection of a Fluid in a Porous Medium." Proceedings of the Cambridge Philosophical Society 44 (1948): 508-521. 
Lasseter, T. J., Withersppon, P. A. and Lippmann, M. J. "Multiphase Multidimensional Simulation of Geothermal Reservoirs." 2nd U.N. Symposium on the Development and Use of Geothermal Resources. San Francisco, CA., U.S.A., 20-29 May, 1975: 1715-1723.

Lasseter, T. J. The Numerical Simultation of Heat and Mass Transfer in MultiDimensional Two-Phase Geothermal Reservoirs. (75-WA/HT-71). New York: American Society of Mechanical Engineers, [1975].

Laughlin, A. W. and others. "Status of the Los Alamos Experiment to Extract Geothermal Energy From Hot Dry Rock." Geology 4 (1977): 237-240.

*Leibowitz, Lewis P. California's Geothermal Resource Potential. (Energy Sources Vo. 3). New York: Crane, Russak \& Co., 1978.

Leverett, M. C. and True, M. E. Dimensional-Model Studies of 0il-Field Behavior. (A.I.M.E. Technical Püblication No. 1413). New York: A.I.M.E., [1942].

Lipman, S. C., Strobel, C. J., and Gulati, M. "Reservoir Performance of the Geysers Field." Proceedings of Larderello Workshop on Geothermal Resource Assessment and Reservoir Engineering (1977): 234-255.

*Lo, Hing Y. and Mungan, N. "Effect of Temperature on Water-0il Relative Permeabilities in 0il-Wet and Water-Wet Systems. Dallas: Society of Petroleum Engineers Paper No. 4505, 1973.

*Lombard, G. L. and Nugent, J. M. "San Diego Gas and Engineering Pioneering Geothermal Test Work in the Imperial Valley of Southern California." Workshop on Geothermal Reservoir Engineering. Stanford: Stanford University Press, 1975.

Lowell, R. P. "Circulation in Fractures, Hot Springs, and Convective Heat Transport on Mid-Ocean Ridge Crests." Geophysical Journal of the Royal Astronomical Society 40 (1975): 351-365.

*Lowell, R. P. "Comments on Theory of Heat Extraction from Fractured Hot Dry Rock by A. C. Gringarten, P. A. Witherspoon, and Yuzo Ohnishi." Journal of Geophysical Research 81 (1976): 359-360.

Mahon, W. A. J. "Chemistry in the Exploration and Exploitation of Hydrothermal Systems." U.N. Symposum on the Development and Utilization of Geothermal Resources, Pisa, Sept. 22-0ct. 1, 1970: 1310-1322.

*Majer, E. and McEvilly, T. V. "The Geysers Geothermal Reservoir Properties from Seismological Data." Geothermal Resources Council Transactions 1, 1977, 199.

Mann, Lloyd. "Well Log Analysis and Well Testing in the Heber Geothermal Field." Workshop on Geothermal Reservoir Engineering. Stanford: Stanford University Press, 1975: 74-76. 
Marconcini R. and others. "Modeling Vapor Dominated Geothermal Reservoirs." Proceedings of the Larderello Workshop on Geothermal Resources Assessment and Reservoir Engineering: 256-298, 1977.

Marshall, D. C. "Development of a Theory of the Wairakei Geothermal Field by the 'Simplest Cases First' Technique." U.N. Symposium on the Development and Utilization of Geothermal Resources. Pisa, Sept. 22-0ct. 1, 1970: 669-676.

Marsha11, D. C. "Preliminary Theory of the Wairakei Geothermal Field." New Zealand Journal of Science 9 (1966): 651-673.

Martin, J. C. "Analysis of Internal Steam Drive in Geothermal Reservoirs." Journal of Petroleum Technology 27 (1975): 1493-1499.

Martin, J. C. "Fluid Flow in Geothermal Reservoirs." Workshop on Geothermal Reservoir Engineering. Stanford: Stanford University Press, 1975: 42-44.

Meidav, Tsvi, Russell, James and Subri, Sanyal. "Utilization of Gravimetric Data for Estimation of Hydrothermal Reservoir Characteristics in the East Mesa Field, Imperial Valley, California." Workshop on Geothermal Reservoir Engineering. Stanford: Stanford University Press, 1975: 52-61.

Meng, C. Y. and Shaw, P. T. "Study of Heat Conduction Models of Geothermal Energy Reservoirs." U.N. Symposium on the Development and Utilization of Geothermal Resources. Pisa, Sept. 22-0ct. 1, 1970: 1270-1274.

Mercado, Sergio. "Movement of the Geothermal Fluids and Temperature Distribution in Cerro Prieto Geothermal Field, Baja, California, Mexico." 2nd U.N. Symposium on the Development and Use of Geothermal Resources. San Francisco, CA., U.S.A., 20-29 May, 1975: 492.

Mercer, James W. Jr. Finite-Element Approach to the Modeling of Hydrothermal Systems. Ph.D. dissertation, University of Illionois, 1973.

Mercer, James W. Jr. and Pinder, G. F. "Galerkin Finite-Element Simulation of a Geothermal Reservoir." Geothermics 2 (Numbers 3-4, 1973): 81-89.

Mercer, James, W. Jr., Pinder, George F. and Donaldson, Ian G. "A GalerkinFinite Element Analysis of the Hydrothermal System at Wairakei, New Zealand." Journal of GeophysicalResearch 80 (1975); 2608-2621.

Mercer, James W. Jr., Faust, Charles R., and Pinder, George F. "Geothermal Reservoir Simulation. " Conference on Research for the Development of Geothermal Energy Resources, September 23-25, 1974: 256-267.

Mercer, James W. Jr. and Faust, Charles R. Simulation of Water-and VaporDominated Hydrothermal Reservoirs. Dallas: Society of Petroleum Engineers Paper No. 5520, 1975.

* Merrill, Lavaun S. Two-Phase Flow in Fracturs. Ph.D. dissertation, University of Denver, 1975. 
*Metzger, S. W. "Geothermal Energy Direct Applications Market-Estimator Model." Geothermal Resource Council Transactions 2 (Part 2, 1978): 437-439.

*Michels, Donald E. " $\mathrm{CO}_{2}$ in Geothermal Steam: A Rapid, Precise, and Accurate Field Assay Technique." Geothermal Resources Council Transactions 2 (Part 2, 1978): 445-448.

*Miller, C. C., Dyes, A. B., and Hutchinson, C. A. Jr. "Estimation of Permeability and Reservoir Pressure from Bottom Hole Pressure Build-Up Cahracteristics." A.I.M.E Transactions 189 (1950): 91-104.

*Miller, E. E. and Miller, R. D. "Physical Theory for Capillary Flow Phenomea." Journal of Applied Physics 27 (1957): 327-332.

Miller, Frank, G. "Steady Flow of Two-Phase Single-Component Fluids Through porous Media." A.I.M.E. Transactions 192 (1951): 205-216.

*Miller, F. G., Prestwich, S. M. and Gould, R. W. "Drilling and Directional Drilling a Moderate-Temperature Geothermal Resource." Geothermal Resource Council Transactions 2 (Part 2, 1978): 445-456.

Miller, Richard J. "Enhanced Recovery Reservoir Engineering Aspects of Geothermal Development." Geothermal Resources Council Transactions 2 (Part 2, 1978): 457-459.

Moench, A. F. and Herkelrath, W. N. "The Effect of Vapor-Pressure Lowering upon Pressure Drawdown and Buildup on Geothermal Steam Wells." Geothermal Resources Council Transactions 2 (Part 2, 1978): 465-468.

Moench, Allen F. Simulation of Steam Transport in Vapor-Dominated Geothermal Reservoirs. (U.S.G.S. Open File Report 76-607). Menlo Park: U.S. Geological Survey, [1976].

*Moore, Thomas V. "Reservoir Engineering Begins Second 25 Years." 0il and Gas Journal 54 (1955): 148-151.

*Mori, Y. "Exploitation of the Matsukawa Geothermal Area." U.N. Symposium on the Development and Utilization of Geothermal Resources. Pisa, Sept. 22-0ct. 1, 1970: 1150-1156.

Morrison, H. L., Horton, C. W. and Rodgers, F. T. Jr. "Convections Currents in Porous Media. II. Observation of Conditions at Onset of Convection." Journal of Applied Physics 20 (1949): 1027-1029.

Morse, John G. and Thorson, Lewis D. "Reservoir Engineering Study of a Portion of the Salton Sea Geothermal Field." Geothermal Resources Council Transactions 2 (Part 2, 1978): 471-474.

Mundry, E. "Mathematical Estimation Concerning the Cooling of a Magmatic Intrustion." U.N. Symposium on the Development and Utilization of Geothermal Resources. Pisa, Sept. 22-0ct. 1, 1970: 662-668. 
*Mura, T., Keer, L. M. and Abe, H. "Analytical Study of Crack Growth and Shape by Hydraulic Fracturing of Rocks." Workshop on Geothermal Reservoir Engineering. Stanford: Stanford University Press, 1975: 180-184.

Murphy, H. D. "Hydraulic-Fracture Geothermal Reservoir Engineering." Workshop on Geothermal Reservoir Engineering. Stanford: Stanford Universtíy Press, 1975, Pp, 174-177.

Murphy, Hugh D. and others. Preliminary Assessment of a Geothermal Energy Reservoir Formed by Hydraulic Fracturing. (LA-UR-76-1672). Los ATamos: Los Alamos Scientific Laboratory, [1976].

*Muskat, Morris. "Use of Data on the Build-Up of Bottom-Hole Pressures." A. I.M.E. Transactions 123 (1937): 44-48.

*McDowe11, G. D. "An Instrument for Measuring the Gas Concentrations in Geothermal Reservoirs." Eleventh Intersociety Conversion Engineering Conference. September 12-17, 1976: 828-835.

McFarland, R. D. and H. D. Murphy. "Extracting Energy from HydraulicallyFractured Geothermal Reservoirs." Eleventh Intersociety Conversion Engineering Conference, September 12-17, 1976: 828-835.

McKee, C. R. and Hanson, M. E. "Predicting Explosion-Generated Permeability Around Geothermal Wells." Workshop on Geothermal Reservoir Engineering Stanford: Stanford University Press, 1975: 192-197.

McNabb, A. Geothermal Physics (D.S.I.R. Technical Report No. 32). Wellington: New Zealand: Department of Scientific and Industrial Research, [1975].

McNabb, A., Grant, M. and Robinson, J. Permeability Estimates. Wellington, New Zealand. Department of Scientific and Industrial Research Technical Publication No. 34, 1975.

McWhorter, D. B. and Corey, A. T. "Similitude for Flow of Two Fluids in Porous Media." International Hydrology Symposium, Fort Collins, Colorado, September, 1967: 18.1-18.5.

Narasimhan, T. N. and Witherspoon, P. A. "Initial Results of Reservoir Production Tests, Raft River Geothermal Project, Idaho." Workshop on Geothermal Reservoir Engineering. Stanford: Stanford University Press, 1975, pp, 124-125.

Nathenson, Manuel. "The Effects of a Step Change in Water Flow on an Initially Linear Profile of Temperature." 2nd Workshop on Geothermal Reservoir Engineering. Stanford: Stanford University Press, 1976.

Nathenson, Manue1. Physical Factors Determining the Fraction of Stored Energy Recoverable from Hydrothermal Convection Systems and Conduction-Dominated Areas. (U.S.G.S. Open File Report 75-525). Men1o Park: U.S. Gelogical Survey, [1975]. 
Nathenson, Manuel. "Reservoir Factors Determining the Fraction of Stored Energy Recoverable from Hydrothermal Convections Systems." Workshop on Geothermal Reservoir Engineering. Stanford: Stanford University Press, 1975, pp. 50-51.

*Neuman, Shlomo P. and Witherspoon, Paul A. "Theory of Flow in a Confined Two Aquifer System." Water Resources Research 5 (1969): 803-816.

*Nicholson, Robert W. "Drilling Fluid Formation Damage in Geothermal Wells." Geothermal Resources Council Transactions 2 (Part 2, 1978): 503-505.

Nogucki, T., Nishikawa, K. and Ushijima, K. "Some Theoretical Considerations on Hydrothermal System Due to Cracks." U.N. Symposium on the Development and Utilization of Geothermal Resources. Pisa, Sept. 22-0ct. 1, 1970: 655-661.

Norton, D. and Knight, J. "A Preliminary Analysis of the Coso Geothermal System." Exploration Criteria for Low Permeability Geothermal Resources, Progess Report, June 1975 - March 1977: 27-53.

*Orszag, Steven A. and Israeli, Moshe. "Numerical Simulation of Viscous Incompressible Flows." Annual Review of Fluid Mechanics 6 (1974): 281319.

*Palmer, T. D. Characteristics of Geothermal Wells Located in the Salton Sea Geothermal Field, Imperial County, California. (University of California UCRL 51976). Livermore: Lawrence Livermore Laboratory, [1975].

Papadopulos, Stavros S. "Energy Potential of Geopressured Reservoirs: Hydrogeologic Factors." Geopressured Geothermal Energy Conference. First, Austin, June 2-4, 1975: 173-192.

*Pearl, Richard Howard. "Hydrological Problems Associated with Developing. Geothermal Energy Systems." Ground Water 14 (1974): 128-137.

Perkins, F. M. and Collins, R. E. "Scaling Laws for Laboratory Flow Models of 0il Reservoirs." Journal of Petroleum Technology 12 (1960): 69-71.

*Pinder, George F. and Frind, Emil 0. "Application of Galerkin's Procedure to Aquifer Analysis." Water Resources Research 8 (1972): 108-120.

Pinder, George F. "The Princeton Geothermal Research Program." Workshop on Geothermal Reservoir Engineering. Stanford: Stanford University Press, 1975: $199-200$.

Piwinski, A. J. and Netherton, R. "Permeability of Kayenta Sandstone to Hypersaline Brine at $10.3 \mathrm{MPa}$ Confining Pressure and Temperature to $90^{\circ} \mathrm{C}$." Stanford Geothermal ReservoirEngineering Workshop, 3rd. Stanford: Stanford University, 1977.

*Podio, Augusto L. and others, "Reservoir Research and Technology." 2nd Geopressured Geothermal Energy Conference, Austin, Texas, February 23-25, 1976: III (1976): $V-1$ to $V-15$. 
Prats, M. "Effect of Horizontal Fluid Flow on Thermally Induced Convection Currents in Porous Mediums." Journal of Geophysical Research 71 (1968): 4835-4838.

*Pratt, H. R. and Simonson, E. R. Geotechnical Studies of Geothermal Reservoirs. (Terra Tek TR 76-2). Salt Lake City: Terra Tek, [1976].

*price, H. S. and Coats, K. H. "Direct Methods in Reservoir Simulation." A. I.M.E Transactions. 257 (1974): 295-308.

Pritchett, J. W. "Numerical Calculation of Multiphase Fluid and Heat Flow in Hydrothermal Reservoirs." Workshop on Geothermal Reservoir Engineering. Stanford: Stanford University Press, 1975: 201-205.

Pritchett, J. W., Garg, S. K., and Riney, T. D. "Numerical Simulation of the Effects of Reinjection upon the Performance of a Geopressured Geothermal Reservoir." Geothermal Resources Council Transactions 1 (1977): 245-247.

R-Rivera, Jesus. "Application of Type Curve Procedures for the Analys is of Production Data from Geothermal We11s." Geothermal Resources Council Transactions 2 (Part 2, 1978): 575-578.

R-Rivera, Jesus. "Decline Curve Analys is--A Useful Reservoir Engineering Tool for Predicting the Performance of Geothermal Wells." Geothermal Resources Council Transactions 1 (1977): 257-259.

*Ramey, Henry J. Jr., Kruger, P and London, A. L. "The Birth of Geothermal Reservoir Engineering." Workshop on Geothermal Reservoir Engineering. Stanford: Stanford University Press, 1975: 20-22.

Ramey, Henry J. Jr. and others. "Geothermal Reservoir Engineering Research." Conference on Research for the Development of Geothermal Energy Resources. September 23-25, 1974: 268-280.

*Ramey, Henry, J. Jr. "Hand Compunter Program for James' LIP Pressure Steam Flow Rate." Geothermal Resources Council Transactions 2 (Part 2, 1978): 555-557.

Ramey, Henry J. Jr. "Pressure Transient Analysis for Geothermal Wells." U.N. Symposium on the Development and Use of Geothermal Resources, San Francisco, May 20-29, 1975: 1749-1757.

* Ramey, Henry J. Jr. Stimulation and Reservoir Engineering of Geothermal Resources. (Stanford Geothermal Program TR-8)... Stanford: Stanford University Press, [1975].

Ramey, Henry J. Jr. and others. Thermodynamic and hydrodynamic Properties of Hydrothermal Systems. (Stanford Geothermal Program TR-6). Stanford: Stanford University Press, [1974].

Ramey, Henry J. Jr. "Wellbore Heat Transmission." Journal of Petroleum Technology 14 (1962): 127-135. 
*Rapoport, L. A. "Scaling Laws for Use in Design and Operation of Water-0il Flow Mode1s." A.I.M.E. Transactions 204 (1955): 143-150.

* Rayleigh, Lord. "On the Convection Currents in a Horizontal Layer of Fluid when the Higher Temperature is on the Under Side." Philosophical Magazine and Journal of Science, Sixth Series 32 (1916): 529-546.

*Reynolds, G. "Cooling with Geothermal Heat." U.N. Symposium on the Development and Utilization of Geothermal Resources. Pisa, Sept. 22-0ct. 1, 1970: 1658-1661.

Ribando, R. J. and Torrance, K. E. Natural Convection in a Porous Medium: Effects of Confinement Variable Permeability and Thermal Boundary Conditions. New York: American Society of Mechanical Engineers Paper No. 76HT-Y, 1976.

Rice, Larry, F." "Pressure Drawdown and Buildup Analysis in Geothermal Reservoirs." Eleventh Intersociety Energy Conversion Engineering Conference. September 12-17, 1976, Stateline, Nevada: 798-801.

Ridgway, Stuart L. "Energetics of Geothermal Fluid Delivery via Two-Phase Flow." Geothermal Resources Council Transactions 1 (1977): 225.

Rinehart, John S. Alterations of Flow Characteristics within Geothermal Areas by Tidal Forces. Springfield: National Technical Information Service, $1975,9 p$.

*Risk, George F. "Monitoring the Boundary of the Broadlands Geothermal Field, New Zealand." 2nd U.N. symposium on the Development and Use of Geothermal Resources. San Francisco, CA, U.S.A., 20-29 May, 1975: 1185-1189.

*Ritchie, J. A. "Chemical Analysis and Laboratory Requirements: Experience in New Zealand's Hydrothermal Areas." Proceedings of U.N. Conference on New Sources of Energy 2 (1964): 361-366.

*Robinson, J. L. "A Note on the Stability of an Infinite Fluid Heated from Below." Journal of Fluid Mechanics 29 (1967): 461-464.

Robinson, John. Modelling of Convective Flows. (D.S.I.R. Report No. 35). Wellington: New Zealand. Deaprtment of Scientific and Industrial Research. Applied Mathematics Division, [1975].

Robinson, Ronald. A Study of the Effects of Various Reservoir Parameters on the Performance of Geothermal Reservoirs. Ph.D. dissertation, Texas A \& M University, 1974.

*Roegiers, J. C. and Brown, D. W. "Geothermal Energy: A New Application of Rock Mechanics," Advances in Rock Mechanics 2A (1974): 674-680.

Roegiers, J. C. and Thill, R. E. "Rock Characterization at a Geothermal Site." 17th International Symposium on Rock Mechanics, Snowbird, Utah. August 25-27, 1976: 5D5-1- 505-6. 
Rogers, F. T. Jr. and Schilberg, L. E. "Convection Currents in Porous Media. III. Extended Theory of the Critical Gradient." Journal of Applied Physics 21 (1950): 1177-1179.

Rogers, F. T. Jr. and Morrison, H. L. "Convection Currents in Porous Media. IV. Remarks on the Theory." Journal of Applied Physics 22 (1951): 14761479.

*Ross, Laurence W. "Solubility of Gases in Fluids Under Geothermal Conditions. Estimation Methods and Example Calculations." Geothermal Resources Council Transactions 1 (1977): 269-270.

*Ross, Laurence W. Two-Phase Flow in Geothermal Energy Sources. Denver: University of Denver, 1976, 24p.

*Rowley, John C. "Rock Melting Technology and Geothermal Drilling." Conference on Research for the Development of Geothermal Energy Resources. September 23,-25, $1974: 239-255$.

*Roy, A. "Depth Investigation in Direct Current Methods.". Geophysics 36 (1971): 943-959.

*Rudisil1, Jacob M. "A Case History: The Completion of a Shallow, Over-Pressured Geothermal We11." Geothermal Resources Council Transactions 2 (Part 2, 1978): 587-590.

Rumi, 0. "Some Observations About the Thermo-Fluid-Dynamic Behavior of the Steam in the Wells of Larderello and About the Best Exploitation Conditions." U.N. Symposium on the Development and Utilization of Geothermal Resources. Pisa, Sept. 22-0ct. 1, 1970: 698-703.

*Ruppel, H. M. A General Numerical Fluid Dynamics Algorithm for Astrophysical Applications. (LA-6149-MS). Los Alamos: Los Alamos Scientific Laboratory, [1975].

*Salisbury, J. W. "ERDA Strategy for Resource Evaluation and Reservoir Confirmation." Proceedings of the Lardere110 Workshop on Geothermal Resource Assessment and Reservoir Engineering. September 12-16, 1977.

*Salisbury, Jack, Williams, D. and Nichols, C. A Position Paper on Resource and Reservoir Assessment and Its Impact on Federal Plans for Development of Geothermal Energy over the Near Term. Washington D.C.: U.S. Energy Research and Development Administration. Division of Geothermal Energy, 1976.

Sammis, Charles G. "Numerical Modeling of Hydrothermal Reactions in Geothermal Reservoirs." Workshop on Geothermal Reservoir Engineering. Stanford:

Stanford University Press, 1975: 240-241.

Sanyal, S. K., and Marsden, S. S. Jr. "Effect of Temperature on Petrophysical Properties of Reservoir Rocks. Dallas: Society of Petroleum Engineers Paper No. 4898, 1974. 
Sarda, Jean-Paul and Hsu, Y.C. Angle of Crack Propagation for a Vertical Hydraul ic Fracture. (LA-6175-MS). Los Alamos: Los Alamos Scientific Laboratory, [1975].

Sato, Ko. and IDE, Toshio. "On the Structural Characters and Simultation of Rock Fracturing of Geothermal Areas in Northeastern Japan." 2nd U.N. Symposium on the Development and Use of Geothermal Resources, San Francisco, CA., U.S.A., 20-29 May, 1975: 575-581.

Scherer, Charles R. "On the Optimal Rate of Geothermal Energy Extraction." Workshop on Geothermal Reservoir Engineering. Stanford: Stanford University Press, 1975: 161-166.

Schrock, V. E. and Laird, A. D. "Physical Modeling of Combined Forced and Natural Convection in Wet Geothermal Formations." Journal of Heat Transfer 98 (1976): 213-220.

Schroeder, R. C. "Reservoir Engineering Report for the Magma-SDG\&E Geothermal Experimental site Near the Salton Sea, California." "University of California UCRL 52094). Livermore, Lawrence Livermore Laboratory, [1976].

Seki, Arthur S., Chen, Bill H., and Takahashi, Patrick K. "Geothermal Reservoir Engineering: Performance Matching and Predicting." American Society of Civil Engineers Journal of the Power Division 104 (1978): 169-181.

Sharma, Devrag and Maini, Tidu. "Geothermal Energy From a Borehole in Hot Dry Rock--A Preliminary Study. Workshop on Geothermal Reservoir Engineering. Stanford: Stanford University Press, 1975: 258-266.

*Shawki, G. S. A. and El-Wahi, S. H. "Strength of Thick-Walled Permeable Cylinders." International Journal of Mechanical Sciences 12 (1970): 535-551.

Shen, H.W. "A Hele-Shaw Model of Heat Convection in Porous Media Under Geothermal Conditions." Workshop on Geothermal Reservoir Engineering. Stanford: Stanford University Press, 1975: 213-218.

*Shirtcliff, T. G. L. "The Development of Layer Thermosolutal Convection." International Journal of Heat and Mass Transfer 12 (1969): 215-222.

*Simmons, Gene, Sigfried, R. W. and Feves, R. "Differential Strain Analysis: A New Method for Examining Cracks in Rocks." Journal of Geophysical Research 79 (1974): 4383-4385.

Sinnokrot, Ali A., Ramey; H. J. and Marsden, S. S. "Effect of Temperature Level Upon Capillary Pressure Curves." Society of Petroleum Engineers Journal 11. (1971): 13-22.

*Snyder, Robert E. "Geothermal Well Completion: State of the Art." Geothermal Resources Council Transactions 2 (Part 2, 1978): 601-603. 
Somerton, W. H. and Mathus, A. K. "Effects of Temperature and Stress on Fluid Flow and Storage Capacity of Porous Rocks." Site Characterization:

17th U.S. Symposium on Rock Mechanics Proceedings, 25-27 August, 1976, Snowbird, Utah: $2 A 2-1--2 A 2-8$.

*Somerton, W. H., El-Shasiani, A. H. and Nobrak, S. M. High Temperature Behavior of Rocks Associated with Geothermal Type Reservoirs. Dallas: Society of Petroleum Engineers Paper No. 4897, 1974.

Sondergeld, Carl H. and Turcotte, D. L. "An Experimental Study of Two-Phase Convection in a Porous Medium with Applications to Geological Problems." Journal of Geophysical Research 82 (1977): 2045-2953.

Sorey, M. L. "Numerical Modeling of Geothermal Systems." Geological Society of America Abstracts with Programs 6 (1974): 962.

Sorey, M. L. Numerical Modeling of Liquid Geothermal Systems. (U.S.G.S. Open File Report 75-613). Menlo Park: U.S. Geological Survey, [1975].

Sorey, M. L. Numerical Modeling of Liquid Geothermal Systems. Ph.D. dissertation, University of California, Berkeley, 1976.

Sorey, M. L. "Research on Numerical Modeling of Liquid Geothermal Systems." Workshop on Geothermal Reservoir Engineering. Stanford: Stanford University Press, 1975: 225-231.

*Souto, Jose, M. "Oahu Geothermal Exploration." Geothermal Resources Council Transactions 2 (Part 2, 1978): 605-607.

Spencer, James W. Jr. and Nur, A. M. "Effects of Pressure Temperature and Pore Water on Velocities in Westerly Granite." Journal of Geophysical Research 81 (1976): 899-904.

Stoker, Alan K. and Kruger, Paul "Radon in Geothermal Reservoirs." U.N. Symposiumon the Development and Use of Geothermal Resources. San Francisco, May 20-29, 1975: 1797-1803.

*Stoker, R. C., Golman, D. and Kunze, J. F. "Deducing Production Zones from Weil Logs." Geothermal Resources Council Transactions 1 (1977): 279-280.

Straus, Joe M., Goldman, D. and Kruger, J. F. "Large Amplitude Convection in Porous Media." Journal of Fluid Mechanics 64 (1974): 51-63.

Straus, Joe M. and Schubert, G. "Thermal Convection of Water in a Porous Medium: Effects of Temperature-and Pressure-Dependent Thermodynamic and Transport Properties." Journal of Geophysical Research 82 (1977): 325-333.

* Swanberg, Chandler A. "The Mesa Geothermal Anomaly, Imperial Valley, California: A Comparison and Evaluation of Results Obtained from Surface Geophysical and Deep Drilling." 2nd U.N. Symposium on the Development and Use of Geothermal Resources. San Francisco, CA., U.S.A., 20-29 May, 1975: $903-907$. 
Takahashi, Patrick K. and Chen, Bill. "Geothermal Reservoir Engineering." Geothermal Energy Magazine 3 (1975): 243257.

Tansev, Erdal 0. and Wasserman, Mel L. "Modeling the Heber Geothermal Reservoir." Geothermal Resources Council Transactions 2 (Part 2, 1978): 645-648.

Tester, Jefferson W. Geothermal Energy from Dry Hot Rock Reservoirs. (LA-UR75-2315). Los Alamos: Los Alamos Scientific Laboratory, [1976].

Tewhey, John D. and others. "Development of Injection Criteria for Geothermal Resources." Geothermal Resources Council Transactions 2 (Part 2, 1978): 649-652.

Tezcan, A. Kenan. "Dry Steam Possibilities in Saraykoy-Kozildere Geothermal Field, Turkey." 2nd U.N. Symposium on the Development and Use of Geothermal Resources. San Francisco, CA., U.S.A., 29-29 May, 1975: 1805-1813.

*Theis, Charles V. "The Relation Between the Lowering of the Piezometric Surface and the Rate and Duration of Discharge of $w$ Well using GroundWater Storage." American Geophysical Union Transactions 16 (1935): 519-525.

*Thomas, L. Kent and Pierson, R. G. "Three-Dimensional Geothermal Reservoir Simulation." Society of Petroleum Engineers Journal 18 (1978): 151-161.

*Thomas, L. Kent and Pierson, R. G. Three Dimensional Geothermal Reservoir Simulation. Dallas: Society of Petroleum Engineers Paper No. 6104, 1978.

Tolivia, Enrique M. "Evaluation of the Geothermal Potential of Cerro Prieto, Baja California (Mexico)." 2nd U.N. Symposium on the Development and Use of Geothermal Resources. San Francisco, CA., U.S.A., 29-29 May, 1975: 279-281.

Tomasson, Jens and Thorsteinsson T. "Use of Injection Packer for Hydrothermal Drillhole Stimulation in Iceland." 2nd U.N. Symposium on the Development and Use of Geothermal Resources, San Francisco, CA., U.S.A., 20-29 May, 1975: 1821-1827.

*Tompkins, Dorothy Campbe11. Power From the Earth: Geothermal Energy. Berkeley: University of California Institute of Governmental Studies, 1972, 30p.

*Toronyi, R. M. Two-Phase, Two-Dimensional Simulation of a Geothermal Reservoir and the Wellbore System. Ph.D. dissertation, Pennsylvania State University, 1974.

Toronyi, R. M. and Faroug Ali, S. M. Two-Phase, Two-Dimensional Simulation of a Geothermal Reservoir. Dallas: Society of Petroleum Engineers Paper No. 5521, 1977. 
*Townsend, A. A. "Temperature Fluctuations over a Heated Horizontal Surface." Journal of Fluid Mechanics 5 (1959): 209-241.

*Truesda11, A. H. and Singers, Wendy. "Calculation of Aquifer Chemistry in Hot-Water Geothermal Systems." Journal of Research of the U.S. Geological Survey 2 (1974): 271-278.

Truesde11, A. H. and White, D. E. "Production of Superheated Steam from Vapor-Dominated Geothermal Reservoirs." Geothermics 2 (Numbers 3-4, 1973): 154-173.

Tsang, Chin Fu and Witherspoon, P, A. "An Investigation of Screening Geothermal Production Wells from Effects of Reinjection." Workshop on Geothermal Reservoir Engineering. Stanford: Stanford University Press, 1975: 62-64.

Tsang, Chin Fu, Lippmann, M. J. and Witherspoon, P. A. "Production and Reinjection in Geothermal Reservoirs." Geothermal Resources Council Transactions 1 (1977): 301-303.

Tsang, Chin Fu and others. "A Study of Alternative Reinjection Schemes for the Cerro Prieto Geothermal Field, Baja California, Mexico," Geothermal Resources Council Transactions 2 (Part 2, 1978): 659-662.

*Tsyrul'nikov, G. N. and others. "Computer Study of Coolant Dynamics in Circulation Systems." Fluid Mechanics 3 (1974): 86-94.

Van Der Knaap, W. "Nonlinear Behavior of Elastic Porous Media." A.I.M.E. Transactions 216 (1959): 179-187.

Van Everdingen, A. F. and Hurst, W. "Application of the Laplace Transformation to Flow Problems in Reservoirs." A.I.M.E. Transactions 186 (1949): 305-324B.

*Veneruso, A. F. and Stoller, H.M. "High Temperature Instrumentation for Geothermal Applications." Geothermal Resources Council Transactions 2 (Part 2, 1978): 679-682.

Wainwright, D. K. "Subsurface and Output Measurements on Geothermal Bores in New Zealand." U.N. Symposium on the Development and Utilization of Geothermal Resources. Pisa, Sept. 22-0ct. 1, 1970: 764-767.

*Warren, Nick. "Characterization of Crack Structure Controlling Permeability using Velocity-Pressure Data." Geothermal Resources Council Transactions 2 (Part 2, 1978): 701-702.

Weertman, J. and others. Heat Extraction from Hot Dry Rock Masses. Progress Report, August 1, 1975-January 31, 1976. (PB-256 775). Evanston: Northwestern University, [1976].

*Weiss, Richard B,, Meidav, Tsvi and 01dknow, Theodora S. "Geochemical-Hydrological Studies of the Imperial Valley, California." Geothermal Resources Council Transactions 1 (1977): 305-307. 
Weres, 0lei and Schroeder, Ronald C. Documentation for Program OGRE. (LBL 7060). Berkeley: Lawrence Berkeley Laboratory, [1978].

*White, Donald E. "Thermal Waters of Volcanic Origin." Geological Society of America Bulletin 68 (1957): 1637-1658.

*White, Walter S. "A Paleohydrologic Model for Mineralization of the White Pine Copper Deporit, Northern Michigan." Economic Geology and the Bulletin of the Society of Economic Geologist 66 (1971): 1-13.

*Whiting, Robert L. and Ramey, Henry J. Jr. "Application of Material and Energy Balances to Geothermal Steam Production." Journal of Petroleum Technology 21 (1979): 898-900.

Wilson, S. H. "Statistical Interpretation of Chemical Results from Drillholes as an Aid to Geothermal Propecting and Exploitation." U.N. Symposium on the Development and Utilization of Geothermal Resources. Pisa, Sept. 220ct. 1, 1970: 1325-1339.

*Witherspoon, P. A. and Neuman, S. P. "Hydrodynamics of Fluid Injection." Underground Waste Management and Environmental Implication. Memoir \#18. Tulsa: American Association of Petroleum Geolgists, 1972.

*Witherspoon, P. A., Narasimhan, T.N. and McEdwards, D. G. "Reservoir Physics." Workshop on Geothermal Reservoir Enginering. Stanford: Stanford University Press, 1975, pp. 4-15.

*Witherspoon, P. A., McEdwards, D. G. and Narsimhan, P.N. Results of Interference Tests from Two Geothermal Reservoirs. Dallas: Society of PetroTeum Engineers Paper No. 6052, 1976.

*Woitke, Laraine J. "The PG and E Geysers Power Plant--A Utili.ty Company's Viewpoint." Workshop on Geothermal Reservoir Engineering. Stanford: Stanford University Press, 1975: 153-156.

Wooding, R. A. "An Approach to Geothermal Development." Workshop on Geothermal Reservoir Engineering. Stanford: Stanford University Press, 1975: 126-129.

Wooding, R. A. "An Experiment on Free Thermal Convection of Water in Saturated Permeable Material." Journal of Fluid Mechanics 3 (1957): 582-600.

Wooding, R. A. Free Convection of Fluid in a Vertical Tube Filled with Porous Material, Journal of Fluid Mechanics 13 (1962): 129-144.

*Wooding, R. A. "Growth of Fingers at an Unstable Diffusing Interface in a Porous Medium or Hele-Shaw Cell." Journal of Fluid Mechanics 39 (1969): 477-495.

Wooding, R. A. "Instability of a Viscous Liquid of Variable Density in a Vertical. Hele-Shaw Cell." Journal of Fluid Mechanics 7 (1960): 501-515. 
*Wooding, R. A. "Methods of Solution of the Equations for Convection in Porous Media, With Geothermal Applications." Workshop on Geothermal Reservoir Engineering. Stanford: Stanford University Press, 1975, pp. 206-212:

*Wooding, R. A. "Mixing-Layer Flows in a Saturated Porous Medium." Journal of Fluid Mechanics 19 (1964): 103-113.

Wooding, R: A. "Rayleigh Instability of a Thermal Boundary Layer in Flow Through a Porous Medium." Journal of Fluid Mechanics 9 (1960): 183-192.

Wooding, R. A. "Steady-State Free Thermal Convection of Liquid in a Saturated Permeable Medium." Journal of Fluid Mechanics 2 (1957): 273-285.

Wooding, R. A. "The Stability of a Viscous Liquid in a Vertical Tube Containing Porous Material." Proceedings of the Royal Society of London 252 (1959): 120-134.

Wooding, R. A. and Grant, M. A. Taupo Geothermal Hydrology. (D.S.I.R. Report No. 22). Wellington: New Zealand. Department of Scientific and Industrial Research. Applied Mathematics Division, [1974].

*Workshop on Environmental Aspects of Geothermal Resources Development, November 20-22, 1974. Sacramento: State of California, Department of Conservation, Division of $0 i 1$ and Gas, 1974, 139p.

*Wyckoff, R. D. and Botset, H. G. "The Flow of Gas-Liquid Mixtures Through Unconsolidated Sands." Physics 7 (1938): 325-345.

*Wyckoff, R. D. and others. "Measurement of Permeability of Porous Media." American Association of Petroleum Geologists Bulletin 18 (1934): 161-190.

Yuen, P. and others. "Preliminary Well Test Results from HGP-A." Geothermal Resources Council Transactions 1 (1977): 309-310.

Yuhara, K. "Estimation of Hydrothermal Systems by Means of Wel1-Head Observations." U.N. Symposium on the Development and Utilization of Geothermal Resources. Pisa, Sept. 22-0ct. 1, 1970: 1458-1462. 



\section{INDEX}

SUBJECT/AUTHOR/YEAR

A

Ana logue, electrical Kononenko, 1974

Analytical solution Atkinson, 1976

Andesite Baba, 1970

Aquifers

Bernard, 1975

Cheng, 1974

Einarsson, 1975

Gringarten, 1975

Hunt, 1970

Isita, 1970

Isokrari, 1976

James, 1965

Kassoy, 1975

$\mathrm{McNabb}, 1975$

Moench, 1978

Scherer, 1975

Tester, 1976

Wooding, 1974

Yuharo, 1970

Aquifers, confined

Aladiev, 1976

Aquifers, unconfined Cheng, 1974

B

\section{Boiling}

Bolton, 1970

Ferrara, 1970

Grant, 1976

Gulati, 1975

Hunsbedt, 1978

Kruger, 1975

Ramey, 1973

Boiling surface

Isherwood, 1977

95

Boreholes

Austin, 1977

Bolton, 1970

PAGE

55

29

9

11

36

82

46

94

50

51

83

52

59

18

69

89

98

24

80

36

92

94

44,45

15

48

55

19

29

92
(A) B

Broadlands

Grant, 1976

44

Cataldi, 1970 93

Donaldson, $1970 \quad 39$

James, $1970 \quad 16$

Mahon, $1970 \quad 95$

Marsha11, $1970 \quad 57$

Sarda, $1975 \quad 88$

Yuhara, $1970 \quad 24$

Boundary conditions

Cheng, $1973 \quad 34$

Donaldson, $1970 \quad 39$

Holst, $1972 \quad 46$

Nathenson, $1976 \quad 96$

Norton, $1977 \quad 64$

Boundary layer

Cheng, 1975

Cheng, $1976 \quad 34$

Wooding, $1960 \quad 77$

Wooding, $1966 \quad 76$

Boussinesq approximation

Straus, 1974

72

Brine

Chen, $1977 \quad 33$

Hinrichs, $1975 \quad 15$

Isita, $1970 \quad 50$

Morse, $1978 \quad 18$

Piwinski, $1977 \quad 19$

Tewhey, $1978 \quad 22$
2.

C

California

Austin, $1977 \quad 29$

Black, $1975 \quad 11$

Chasteen, $1974 \quad 81$

Hinrichs, $1975 \quad 15$

Kassoy, $1976 \quad 53$

Mann, $1975 \quad 17$

Meidav, $1975 \quad 18$

Morse, 1978 18

Sorey, $1974 \quad 71$

Tansev, $1978 \quad 73$

Truesde11, $1973 \quad 74$ 
SUBJECT/AUTHOR/YEAR

PAGE

Cap rock

Cheng, 1975

Tezcan, 1975

Truesde11, 1973

Carbon dioxide

Glover, 1970

Kuwada, 1975

Cerro Prieto

James, 1970

R-Rivera, 1977

R-Rivera, 1978

Chemical deposition

Bolton, 1973

Meidav, 1975

Chemistry

Calamai, 1973

Chimney model

Ramey, 1975

Compaction

Ribando, 1976

35

74

74

94

85

16

96

96

80

18

81

66

66

Composition

Isokrari, 1976

51

Compressibility

Chen, 1975

13

Van der Knapp, 1959

23

Conduction

Garg, 1974

43

Gringarten, 1973

Gringarten, 1975

Meng, 1970

Mundry, 1970

Murphy, 1975

McFarland, 1976

Nathenson, 1975

Robinson, 1974

Wooding, 1957

Conduction dominated reservoirs Nathenson, 1975

Conductive heat loss Lowe11, 1975.
SUBJECT/AUTHOR/YEAR

PAGE

Conductivity

Bodvarsson, 1977

Papadopulos, $1975 \quad 87$

Conductivity, thermal

Donaldson, 1970

Donaldson, 1970

39

Dudarev, $1970 \quad 40$

Meng, $1970 \quad 60$

Ramey, $1974 \quad 20$

Wooding, $1957 \quad 76$

Convection

Bories, 1973

Browne 1 1, $1975 \quad 32$

Chen, $1977 \quad 33$

Cheng, 1974

Donaldson, $1970 \quad 39$

Garg, 1974

Gringarten, $1973 \quad 45$

Gringarten, $1976 \quad 45$

Holst, $1972 \quad 46$

McNabb, $1975 \quad 59$

Meidav, $1975 \quad 18$

Morrison, 1949

Murphy, $1975 \quad 86$

Nathenson, $1975 \quad 96$

Ribando, $1976 \quad 66$

Robinson, $1974 \quad 67$

Rogers, $1950 \quad 68$

Rogers, $1951 \quad 68$

Schrock, $1976 \quad 69$

Shen, $1975 \quad 69$

Sorey, 1975

Wooding, 1957

Wooding, $1957 \quad 76$

Wooding, 1964

Convection, forced

Bodvarsson, $1969 \quad 30$

Cheng, $1975 \quad 34$

Combarnous, $1971 \quad 37$

Gringarten, $1975 \quad 46$

Hardee, $1975 \quad 46$

Kassoy, $1975 \quad 52$

Convection, free

Cheng, $1974 \quad 36$

Cheng, $1975 \quad 35$

Cheng, $1975 \quad 34$

Cheng, $1976 \quad 35$ 


\begin{tabular}{|c|c|}
\hline BJECT/AUTHOR/YEAR & PAGE \\
\hline $\begin{array}{l}\text { Convection, free (cont.) } \\
\text { Cheng, 1976 } \\
\text { Combarnous, 1971 } \\
\text { Elder, 1960 } \\
\text { Elder, 1967 } \\
\text { Hardee, 1976 } \\
\text { Holst, 1972 } \\
\text { Holst, 1972 } \\
\text { Horton, 1945 } \\
\text { Kassoy, 1975 } \\
\text { Kassoy, 1975 } \\
\text { Lapwood, 1948 } \\
\text { Straus, 1974 } \\
\text { Wooding, 1962 }\end{array}$ & $\begin{array}{l}34 \\
37 \\
40 \\
41 \\
46 \\
46 \\
46 \\
48 \\
52 \\
52 \\
56 \\
72 \\
76\end{array}$ \\
\hline
\end{tabular}

Convection, nonsteady state Lowe 11, 1975

57

Convection, steady state Lowe 11, 1975

57

Convection, thermal

Combarnous, 1975

Dona Idson, 1962

Robinson, 1975

Sondergeid, 1977

Straus, 1977

Wooding, 1957

$\underline{D}$

Darcy's Law

Bredehoeft, 1965

Wooding, 1957

Decline-curve analysis

R-Rivera, 1978

Density

Lasseter, 1975

Diffusion

Wooding, 1957

Diffusion, heat

Horne, 1974

Ramey, 1974

Diffusivity

Delisle, 1975

Horne, 1974

38

40

67

70

73

77

32

77

96

56

47

20

14

47
SUBJECT/AUTHOR/YEAR

PAGE

Diffusivity (cont.)

Mundry, 1970

Wooding, 1959

77

Discharge

Donaldson, $1962 \quad 40$

Einarsson, $1975 \quad 82$

Elder, $1967 \quad 41$

Grant, no date $\quad 44$

Grant, $1976 \quad 45$

Horne, $1978 \quad 15$

James, $1970 \quad 16$

Moench, $1978 \quad \ldots \quad 18$

Discharge, field

Bolton, 1970

92

Dispersion Wooding, 1957

76

Downhole measurements

Bolton, 1973

80

Drawdown

Campbe11, 1978

Grant, 1976

James, 1970

Marshal1, 1970

Marsha11, 1960

Nathenson, 1976

81

44,45

16

57

58

96

Dritlholes

Tomasson, $1975 \quad 89$

Wilson, 1970

97

Dritling

Baba, $1970 \quad 9$

Celati, 1975

12

Kunze, $1975 \quad 16$

Laughi in, $1977 \quad 85$

Tolivia, 1975

97

E

Earth tides

Narasimhan, $1975 \quad 19$

El Salvador

Einarsson, 1975

82

Elastic modulus

Van der Knapp, 1959 


\begin{tabular}{|c|c|c|c|}
\hline AUTHOR/SUBJECT/YEAR & PAGE & AUTHOR/SUBJECT/YEAR & PAGE \\
\hline $\begin{array}{l}\text { Elasticity } \\
\quad \text { Bodvarsson, } 1977 \\
\text { Endoclaves } \\
\quad \text { Dudarev, } 1970 \\
\text { Energy balance } \\
\quad \text { Isherwood, } 1977\end{array}$ & 40 & $\begin{array}{l}\text { Finite difference } \\
\text { Cheng, 1974 } \\
\text { Coats, 1977 } \\
\text { Donaldson, } 1970 \\
\text { Donaldson, 1962 } \\
\text { Faust, 1976 } \\
\text { Marshal1, 1970 } \\
\text { Meng, 1970 } \\
\text { Mundry, 1970 }\end{array}$ & $\begin{array}{l}36 \\
36 \\
39 \\
40 \\
41 \\
57 \\
60 \\
63\end{array}$ \\
\hline $\begin{array}{l}\text { Energy loss } \\
\text { Ramey, } 1962 \\
\text { Energy potential } \\
\text { Bolton, } 1973 \\
\text { Energy transport } \\
\text { Knapp, } 1977 \\
\text { Mercer, } 1974\end{array}$ & $\begin{array}{l}54 \\
61\end{array}$ & $\begin{array}{l}\text { Finite element } \\
\text { Bazant, } 1975 \\
\text { Chen, } 1977 \\
\text { Faust, } 1975 \\
\text { Faust, } 1976 \\
\text { Mercer, } 1975 \\
\text { Sato, } 1975 \\
\text { Tsang, } 1977\end{array}$ & $\begin{array}{r}29 \\
33 \\
43 \\
41,42 \\
61 \\
88 \\
75\end{array}$ \\
\hline $\begin{array}{l}\text { Enthalpy } \\
\text { Assens, } 1976 \\
\text { Glover, 1970 } \\
\text { Grant, no date } \\
\text { Grant, 1976 } \\
\text { Horne, } 1978 \\
\text { Mahon, 1970 } \\
\text { Nathenson, } 1975 \\
\text { Yuhara, 1970 } \\
\text { Equilibrium continuity } \\
\text { Assens, 1976 }\end{array}$ & $\begin{array}{l}28 \\
94 \\
44 \\
45 \\
15 \\
95 \\
87 \\
24\end{array}$ & $\begin{array}{l}\text { Fissures } \\
\text { James, } 1970 \\
\text { Sato, } 1975 \\
\text { Flash steam } \\
\text { James, } 1965 \\
\text { Flow. } \\
\text { Gould, } 1974 \\
\text { James, } 1970 \\
\text { Leverett, } 1942 \\
\text { Robinson, } 1975\end{array}$ & $\begin{array}{l}82 \\
16 \\
57 \\
67\end{array}$ \\
\hline $\begin{array}{l}\text { Exploitation } \\
\text { Bolton, } 1973 \\
\text { Chasteen, } 1974 \\
\text { Takahashi, } 1975 \\
\text { Wilson, } 1970\end{array}$ & $\begin{array}{l}80 \\
81 \\
73 \\
97\end{array}$ & $\begin{array}{l}\text { Flow doublet } \\
\text { Tsang, } 1978 \\
\text { Flow field } \\
\text { Kononenko, } 1974 \\
\text { Nathenson, } 1975\end{array}$ & $\begin{array}{l}55 \\
87\end{array}$ \\
\hline $\begin{array}{l}\text { Exploration } \\
\text { Bodvarsson, } 1970 \\
\text { Bolton, 1970 } \\
\text { Bolton, 1973 } \\
\text { Donaldson, } 1970 \\
\text { Truesde11, } 1973 \\
\text { F } \\
\text { Faults } \\
\text { Ferrara, } 1970 \\
\text { Kassoy, } 1976 \\
\text { Lowe11, } 1975\end{array}$ & $\begin{array}{l}80 \\
92 \\
80 \\
39 \\
74\end{array}$ & $\begin{array}{l}\text { Flow, fluid } \\
\text { Bazant, 1975 } \\
\text { Bodvarsson, } 1969 \\
\text { Cheng, 1974 } \\
\text { Garg, 1978 } \\
\text { Hanson, 1978 } \\
\text { Kasameyer, 1975 } \\
\text { Martin, 1975 } \\
\text { Mercer, 1975 } \\
\text { Ridgway, 1977 } \\
\text { Somerton, 1976 } \\
\text { Weertman, 1976 }\end{array}$ & $\begin{array}{l}29 \\
30 \\
36 \\
15 \\
83 \\
52 \\
59 \\
60 \\
88 \\
70 \\
90\end{array}$ \\
\hline
\end{tabular}


SUBJECT/AUTHOR/YEAR

Flow, heat

Lapwood, 1948

Meidav, 1975

Mercer, 1973

Ramey, 1962.

Rogers, 1950

Tester, 1976

Flow, isothermal

Brigham, 1975

Kassoy, 1975

Martin, 1975

Flow, mass

Donaldson, 1968

Donaldson, 1970

Ferrara, 1970

Kassoy, 1975

Flow meter

Kubota, 1975

Flow, non isothermal Atkinson, 1976 Garg, 1978 Hunsbedt, 1976

Flow properties Meidav, 1975

Flow rates

Atkinson, 1976

Cataldi, 1970

Murphy, 1975

Nathenson, 1976

R-Rivera, 1977

Rumi, 1970

Yuen, 1977

Flow, single phase

Atkinson, 1976

Bredehoeft, 1965

Brownel1, 1975

Coats, 1977

Faust, 1975

Faust, 1976

Grant, 1976

Kasameyer, 1977

Lasseter, 1975

Pritchett, 1975

Rice, 1976

Sorey, 1974

Tansev, 1978
PAGE

56

18

60

20

68

89

32

52,53

59,96

39

39

94

52

84

29

14

49

18

29

93

86

96

96

21

24

29

32

32

36

43

42

44

84

56

65

20

71

73
SUBJECT/AUTHOR/YEAR

PAGE

Flow, steam/water

Averiev, 1964

Donaldson, 1968

Flow, two-phase

Atkinson, 1977

Averiev, 1964

Barel1i, 1975

Brigham, 1975

Brigham, 1976

Brownel1, 1975

Cady, 1972

Celati, 1977

Chen, 1977

Chen, 1978

Cheng, 1974

Coats, 1977

Faust, 1975

Faust, 1976

Garg, 1978

Gould, 1974

Grant, no date

Grant, 1976

Gulati, 1975

Isokrari, 1976

Juprasert, 1977

Lasseter, 1975

Martin, 1975

Pritchett, 1975

Rice, 1976

Ridgway, 1977

Wainwright, 1970

92

51

84

56

59,96

65

20

88

23

Flow, water

Mahon, 1970

95

Flow, well

Rumi, 1970

Fluid circulation

Hunsbedt, 1977

48

Fluid density Wooding, 1957

77

Fluid expansion

Isokrari, 1976

51

Fluid injection

Bodvarsson, $1977 \quad 31$

Hinrichs, $1975 \quad 15$

Ramey, 1962 
SUBJECT/AUTHOR/YEAR

Fluid loss

Bodvarsson, 1977

Fluid properties

Isokrari, 1976

Ramey, 1974

Fluid, vapor-dominated Miller, 1978

Fluid withdrawal

Cheng, 1973

Grant, no date

Hunsbedt, 1977

Isherwood, 1977

Fluids

Cady, 1969

Wainwright, 1970

Wooding, 1962

Formation permeability

Chen, 1978

Fracture surfaces

Rinehart, 1975

Fractures

Atkinson, 1977

Batzle, 1977

Bazant, 1975

Bodvarsson, 1974

Bodvarsson, 1977

Coats, 1977

Delisle, 1975

Demarest, 1975

Ferrara, 1970

Gringarten, 1973

Hsu, 1975

Hsu, 1978

Hunsbedt, 1976

Lowe 11, 1975

McKee, 1975

Sarda, 1975

Fumaroles

Hunt, 1970

Fusion

Chen, 1978
PAGE

31

54

19

86

34

44

48

95

33

23

76

13

67

9

11

29

30

30

36

14

14

94

45

15

83

48

57

85

88

94

82
SUBJECT/AUTHOR/YEAR

PAGE

$\underline{G}$

Galerkin method

Bazant, 1975

29

Faust, 1976

Straus, 1974

42

72

Gas

Grant, 1976

44

Isokrari, 1976

Gas concentration

$$
\begin{array}{ll}
\text { Ellis, } 1964 \\
\text { Glover, } 1970 \\
\text { Grant, } 1976 .
\end{array}
$$

93

94

44

Gas expansion

McKee, 1975

85

Gas saturation

Isokrari, 1976

Knapp, 1977

51

54

Geochemistry

Laughl in, $1977 \quad 85$

Mahon, $1970 \quad 95$

Sammis, $1975 \quad 68$

Weertman, $1976 \quad 90$

Geothermal anomalies

Kassoy, 1975

52

Geothermal areas

Lowe11, 1975

57

Geotherma 1 energy

Bodvarsson, $1970 \quad 80$

Cady, 1969

Wilson, $1970 \quad 97$

Geothermal fields

Bolton, 1973

Cataldi, 1970

80

93

Geothermal gradient

Sharma, 1975

89

Geothermal power

James, 1978

51 
SUBJECT/AUTHOR/YEAR

Geysers

Budd, 1973

Lipman, 1977

Truesde11, 1973

Ghyben Herzberg lens Cheng, 1973

Granite

Murphy, 1976

Spencer, 1976

Gravity

Hunt, 1970

Isherwood, 1977

Gravity acceleration Wooding, 1957.

Gravity anomalies

Meidav, 1975

Gravity segregation

Martin, 1975

58,96

Groundwater

Black, 1975

Isita, 1970

Kubota, 1975

Robinson, 1975

Schrock, 1976

Wooding, 1975

Groundwater systems

Faust, 1975

Marsha 11, 1966

Gulf Coast

Isokrari, 1976

H

Hawati

$$
\begin{aligned}
& \text { Chen, } 1978 \\
& \text { Cheng, } 1973 \\
& \text { Cheng, } 1976 \\
& \text { Yuen, } 1977
\end{aligned}
$$

Heat
18

51

81.

95
74

34

86

72

94

95

77

11

50

84

67

69

75

42

58

30
SUBJECT/AUTHOR/YEAR

PAGE

Heat (cont.)

Chen, 1978

Cheng, 1976

Donaldson, 1970

Mundry, 1970

82

34

39

63

Heat balance

Assens, 1976

Sharma, 1975

28

89

Heat capacity

Ramey, 1974

Weertman, 1976

20

90

Heat exchange

Balagna, $1976 \quad 10$

Chen, 1978

82

Mahon, 1970

95

Heat extraction

$\begin{array}{ll}\text { Bazant, 1975, } & 29 \\ \text { Bodvarsson, 1974 } & 30 \\ \text { Bodvarsson, 1977 } & 31 \\ \text { Hardee, 1976 } & 46 \\ \text { Kruger, 1975 } & 55 \\ \text { Weertman, 1976 } & 90\end{array}$

Heat flux

Horne, 1974

Sondergeld, 1977

47

70

Heat recovery

Miller, 1978

86

Robinson, 1974

67

Heat, residual

Einarsson, $1975 \quad 82$

Heat storage

Bodvarsson, $1977 \quad 31$

Heat transfer

- Aladiev, $1976 \quad 80$

Atkinson, $1976 \quad 29$

Bodvarsson, $1969 \quad 30$

Bories, $1973 \quad 31$

Chen, $1977 \quad 33$

Cheng, $1976 \quad 35$

Combarnous, $1971 \quad 37$

Dudarev, $1970 \quad 40$

Gouid, $1974 \quad 82$

Gringarten, $1973 \quad 45$ 
Heat transfer (cont.)

Gulati, 1975

Hardee, 1976

Holst, 1972

Hsu, 1978

83

Hunsbedt, 1977

Juprasert, 1977

Kassoy, 1975

84

Kassoy, 1976

Kuo, 1975

Lasseter, 1975

McFarland, 1976

Ramey, 1962

Schrock, 1976

Sharma, 1975

Sorey, 1975

Heat transport

McNabb, 1975

Hele-Shaw cell

Combarnous, 1975

38

Elder, 1967

Shen, 1975

Wooding, 1969

69

76

Horner method

Barelli, 1975

Ramey, 1975

Hot dry rock

Aamodt, 1973

80

Balagna, 1976

Coats, 1977

Delisie, 1975

Diadkin, 1975

Gringarten, 1973

Hsu, 1975

Kruger, 1975

Laughlin, 1977

McFarland, 1976

Murphy, 1975

Norton, 1977

Roegiers, 1976

Tester, 1976

Hot springs

Lowell, 1975

Sorey, 1975

Wooding, 1974

Hot water

Kubota

Hydrautic fracturing

$$
\text { Aamodt, } 1973 \quad 80
$$

Bazant, $1975 \quad 29$

Delisle, $1975 \quad 14$

Hsu, $1978 \quad 83$

Hunsbedt, $1977 \quad 48$

Kunze, $1975 \quad 16$

Laughi in, $1977 \quad 85$

Murphy, $1976 \quad 86$

Tester, $1976 \quad 89$

Weer tman, $1976 \quad 90$

Hydraulics

Tolivia, $1975 \quad 23,97$

Hydrogeology

Faust, 1975

Hydrogeothermal systems

Donaldson, $1968 \quad 39$

McNabb, $1975 \quad 59$

Hydrology

Atkinson, $1977 \quad 9$

Banwel1, $1964 \quad 10$

Barelli, $1975 \quad 10$

Black, $1975 \quad 11$

Ramey, $1975 \quad 20$

Hydrothermics

Cheng, $1976 \quad 34$

Donalds on, $1968 \quad 39$

James, $1965 \quad 83$

Hysteresis

Elder, 1967

I

Idaho

Kunze, $1975 \quad 16$

Imperial Valley

Tansev, $1978 \quad .73$

Weertman, $1976 \quad 90$

Injection

Arihara, $1974 \quad 28$

Calamai, 1973

Chasteen, $1974 \quad 81$

Cheng, $1975 \quad \because \quad 35$

Coats, $1974 \quad 37$ 
SUBJECT/AUTHOR/YEAR

Injection (cont.)

Einarsson, 1975

Gringarten, 1975

Hunsbedt, 1977

Kasameyer, 1977

Kunze, 1976

Mann, 1975

Murphy, 1975

Pinder, 1975

Robinson, 1974

Scherer, 1975

Schroeder, 1976

Tewhey, 1978

Tomasson, 1975

Tsang, 1977

Internal energy

Assens, 1970

Intrusion

Cheng, 1976

Mundry, 1970

Sato, 1975

Investigation bores

Banwe11, 1964

Isotherms

Aladiev, 1976

Cheng, 1973

Isotope analysis

Celati, 1973

Italy

Atkinson, 1977

Barel1i, 1970

Barelli, 1975

Bolton, 1973

Cataldi, 1970

Celati, 1973

Celati, 1975

Celati, 1977

Ferrara, 1970

James, 1968

Marconcini, 1977

Rumi, 1970

Truesde11, 1973

Weres, 1978
PAGE

82

46

48

84

16

17

86

64

67

69

21

22

89

75

28

35

63

88

10

80

34

93

92

10

11

80

93

93

12

33

94

51

59

21

74

75
SUBJECT/AUTHOR/YEAR

PAGE

J

Japan

$\begin{array}{lr}\text { Baba, } 1970 & 9 \\ \text { Kubota, } 1975 & 84 \\ \text { Nogucki, 1970 } & 64 \\ \text { Truesdei1, 1973 } & 74\end{array}$

Jet flows

Donaldson, $1970 \quad 39$

$\underline{L}$

La Place transformation Mercer, 1973

Ramey, 1975

61

20

Larderel10

Bolton, 1973

80

Linear theory

Leverett, 1942

57

Lithology

Tansev, 1978

73

Logging

Baba, 1970

Kunze, 1976

Laughi in, 1977

Roegiers, 1976

Schroeder, 1976

9

16

85

20

21

Los Alamos

Laught in, 1977

85

M

Mass transfer

Aladiev, $1976 \quad 80$

Austin, $1977 \quad 29$

Dudarev, $1970 \quad 40$

Kassoy, $1975 \quad 52$

Kassoy, $1976 \quad 53$

Kuo, $1975 \quad 55$

Lasseter, $1975 \quad 56$

McFarland, $1976 \quad 85$ 
SUBJECT/AUTHOR/YEAR

Mass transport

Glover, 1970

McNabb, 1975

Wooding, 1960

Mathematical models

Atkinson, 1976

Foster, 1965

Gringarten, 1976

Mexico

Isita, 1970

James, 1970

R-Rivera, 1978

Model, steady-state

Mercer, 1973

Modeling

Cady, 1969

Norton, 1977

Weertman, 1976

Modeling, analytical

Takahashi, 1975

Modeling, computer

Norton, 1977

Takahashi, 1975

Modeling, reservoir

Bazant, 1975

Kunze, 1975

Models, convective

Donaldson, 1970

Models, linear flow

Arihara, 1974

Bredehoeft, 1965

Leverett, 1942

Ramey, 1975

Models, numerical

Cheng, 1970

Cheng, 1973

Cheng, 1974

Cheng, 1976

Delisie, 1975

Elder, 1967

Holst, 1972

Hunsbedt, 1976
PAGE

94

59

76

29

59

45

50

16

96

60

33

64

90

73

64

73

29

16

39

28

32

57

66

34

34

36

35

14

41

46

49
SUBJECT/AUTHOR/YEAR

PAGE

Models, numerical (cont.)

$\begin{array}{ll}\text { Marconcini, } 1977 & 59 \\ \text { R-Rivera, 1978 } & 96 \\ \text { Robinson, 1974 } & 67 \\ \text { Sorey, 1974 } & 71 \\ \text { Takahashi, 1975 } & 73 \\ \text { Toronyi, 1977 } & 74\end{array}$

Models, physical

Brigham, $1975 \quad 32$

Elder, $1967 \quad 41$

Hunsbedt, $1978 \quad 48$

Kruger, $1975 \quad 55$

Schrock, $1976 \quad 69$

Takahashi, 1975

73

Models, transient

Mercer, 1973

60

Models, two-dimensiona 1

Bodvarsson, 1969

Chen, 1977

30

Cheng, 1974

$\underline{N}$

New Zealand

Bolton, 1970

Bolton, $1973 \quad 80$

Donaldson, $1970 \quad 39$

Donaldson, $1977 \quad 38$

Ellis, $1964 \quad 93$

Faust, 1975

Gould, $1974 \quad 82$

Grant, $1976 \quad 44,45$

Horne, $1978 \quad 15$

Hunt, $1970 \quad 94$

James, $1965 \quad 83$

James, $1968 \quad 51$

Mahon, $1970 \quad 95$

Marsha 11, $1966 \ldots 58$

Marsha 11, $1970 \quad 57$

Mercer, $1973 \quad 60$

McNabb, $1975 \quad 17$

Pritchett, $1975 \quad 65$

Robinson, $1975 \quad 67$

Wainwright, $1970 \quad 23$

Wooding, $1957 \quad 75,77$

Wooding, 1960

Wooding, $1974 \quad 98$ 
SUBJECT/AUTHOR/YEAR

PAGE

Nonlinear partial differential equations

Cheng, 1974

36

Nusse1t number

Horne, 1974

Shen, 1975

Wooding, 1957

69

77

$\underline{0}$

Ocean ridges

Lowe11, 1975

57

$0 i 1$

Ramey, 1975

20

Seki, 1978

97

$\underline{p}$

$P / Z$ behavior

Atkinson, 1977

Percolation

Isita, 1970

50

Permeability

Aladiev, 1976

80

Arihara, 1976

Atkinson, 1976

Austin, 1977

Balagna, 1976

Bare11i, 1975

Bodvarsson, 1975

Bories, 1973

Brigham, 1975

Cady, 1972

Coats, 1977

Delisie, 1975

Diadkin, 1975

Donaldson, 1968

Dudarev, 1970

Faust, 1975

Horton, 1945

Hunsbedt, 1978

Isita, 1970

James, 1970

Kasameyer, 1975

Kuwada, 1975

Leverett, 1942

92
SUBJECT/AUTHOR/YEAR

PAGE

Permeability (cont.)

Mann, 1975

Marshall, 1968

Meidav, 1975

McKee, 1975

McNabb, 1975

Morse, 1978

Murphy, 1977

Nathenson, 1975

Piwinski, 1977

Ramey, 1975

Ribando, 1976

Rice, 1976

Rinehart, 1975

Sammis, 1975

Somerton, 1976

Tester, 1976

Tezcan, 1975

Tolivia, 1976

Toronyi, 1977

Wooding, 1957

Wooding, 1975

Perturbation

Bodvarsson, $1969 \quad 30$

Cheng, 1976 34

Wooding, 1957

77

Perturbation analys is

Cheng, 1975

35

Poisson's ratio

Hsu, 1978

83

Roegiers, $1976 \quad 20$

Spencer, $1976 \quad 72$

Pollution

Bolton, $1973 \quad 80$

Kubota, $1975 \quad 84$

Pore pressure

Murphy, $1977 \quad 86$

Rinehart, $1975 \quad 67$

Porosity

Barelli, $1975 \quad 11$

Brigham, $1977 \quad 92$

Cady, $1972 \quad 33$

Chen, $1974 \quad 13$

Coats, $1977 \quad 36$

Hanson, $1978 \quad 83$

Hunsbedt, $1976 \quad 49,50$ 
Porosity (cont.) Hunsbedt, 1978

Porous rocks

Mann, 1975

Moench, 1978

Nathenson, 1975

Ramey, 1974

Rinehart, 1975

Roegiers, 1976

67

Sammis, 1975

20

Somerton, 1976

68

Tolivia, 1976

Toronyi, 1977

Porous equation

Elder, 1967

Power, electrical

Chasteen, 1974

Donaldson, 1977

Hunsbedt, 1977

38

Isokrari, 1976

48

51

Power generation

Bolton, 1970

Chasteen, 1974

92

Hunsbedt, 1977

81

Isokrari, 1976

48

Lipman, 1977

51

95

Porous materials

Toronyi, 1977

Wooding, 1959

Wooding, 1962

Porous media

Arihara, 1974

Atherton, 1975

Atkinson, 1976

Atkinson, 1977

Baba, 1970

Bodvarsson, 1975

28

29

Bories, 1973

30

Chen, 1977

Chicoine, 1977

Combarnous, 1975

Power production

Budd, 1973

Hanson, 1978

81

Mercer, 1974

83

61

Precipitation

Kuwada, 1975

85

Prediction

R-Rivera, $1978 \quad 96$

Seki, $1978 \quad 97$

Pressure

Banwel1, $1964 \quad 10$

Bodvarsson, $1975 \quad 30$

Calamai, $1973 \quad 81$

Cataldi, $1970 \quad 93$

Einarsson, $1975 \quad 82$

Garg, $1978 \ldots 14$

Horne, $1978 \quad 15$

James, $1970 \quad 16$

Moench, $1976 \quad 62$

Ramey, $1975 \quad 19$

Scherer, $1975 \quad 69$

Spencer, $1976 \quad \therefore 72$

Wooding, $1.974 \quad 98$

Nogucki, 1970

Prats, 1966

Rogers, 1950

Rogers, 1951

Sinnokrot, 1971

Sorey, 1974.

Sorey, 1975

Straus, 1974

Toronyi, 1977

Van der Knapp, 1959

Wooding, 1957

Wooding, 1960

Pressure buildup

Barel1i, 1970

Barel1i, 1975

10

Chen, 1978

Mann, 1975

Rice, 1976

Pressure, capillary

Atkinson, 1977

Chicoine, 1977 
SUBJECT/AUTHOR/YEAR

PAGE

Pressure, capillary (cont.) Sinnokrot, 1971 70

Pressure distribution

$$
\text { Celati, } 1975
$$

Pressure downhole Glover, 1970

Hinrichs, 1975

Pressure drawdown

Bolton, 1970

Brigham, 1977

Donaldson, 1977

92

92

38

Ferrara, 1970

Gulati, 1975

James, 1970

Lipman, 1977

Moench, 1978

Rice, 1974

Pressure field

Kononenko, 1974

55

Pressure, fluid

Sarda, 1975

Pressure, formation

Celati, 1976

Pressure, high

Budd, 1973

Pressure, over-burden

Sarda, 1975

88

Pressure, steam

Celati, 1977.

33

Pressure transients

Bare11i, 1975

Celati, 1975

Donaldson, 1977

Gulati, 1975

Narasimhan, 1975

Pressure, vapor

Cady, 1972

Chicoine, 1977

Moench, 1978

11,29

12

38

15

19

33

36

18

Pressure, well thead

Einarsson, 1975

88

12

81

82
SUBJECT/AUTHOR/YEAR

PAGE

Productivity

Campbe11, $1978 \quad 81$

Miller, 1978

Production

Kassameyer, $1977 \quad 84$

Toronyi, $1977 \quad 74$

Tsang, 1977

75

Production, finite element Pinder, 1975

64

Production, oil

Budd, 1973

81

Production simulator

Toronyi, 1977

74

Pumping

Campbe11, 1978

81

Pumps, downhole

Kuwada, 1972

85

Quartz thermometry

Kuo, 197.5

55

R

Radon

Stoker, 1975

22

Raft River

Kunze, $1975 \quad 16$

Narasimhan, 1975

Rayleigh number

Bories, 1973

Cheng, 1973

31

34

Cheng, $1975 \quad 35$

Cheng, $1976 \quad 34,35$

Combarnous, $1971 \quad 37$

Donaldson, $1970 \quad 39$

Elder, $1967 \quad 41$

Holst, $1972 \quad 46$

Horton, $1945 \quad 48$

Kassoy, $1975 \quad 53$

Prats, $1966 \quad 65$

Shen, 1975

69 
AUTHOR/SUBJECT/YEAR

Rayleigh number (cont.)

Straus, 1977

Wooding, 1957

Wooding, 1960

Recharge

Barelli, 1975

Cataldi, 1970

Donaldson, 1962

Donaldson, 1977

Grant, 1976

Hunsbedt, 1976

Knapp, 1977

Miller, 1978

Morse, 1978

Nathenson, 1976

Robinson, 1974

Tolivia, 1976

Recharge fluid

Cataldi, 1970

Nathenson, 1975

Recharge rate

Isherwood, 1977

Recovery, enhanced

Miller, 1978

Recovery, oil

Ramey, 1962

Reinjection

Einarsson, 1975

Hanson, 1978

Knapp, .1977

Kubota, 1975

Tsang, 1977

Relative permeability Ramey, 1974

Relaxation methods Wooding, 1957

Reservoir boundaries

Chen, 1974

Narasimhan, 1975

Reservoir engineering

Atkinson, 1977

Bernard, 1975
20

PAGE

73

77

76,77

11

93

40

38

45

49

54

62

18

96

67

23

93

87

95

86

20

82

83

54

84

75

77

13

19

9
AUTHOR/SUBJECT/YEAR

PAGE

Reservoir engineering (cont.)

Garg, 1978

Miller, 1978

Seki, 1978

Weres, 1978

62,86

97

75

Reservoir exploitation

Celati, 1973

93

Reservoir fluids

Cady, 1969

33

Miller, 1978

86

Sanya 1, 1974

21

Reservoir, fractured

$$
\text { Celati, } 1977
$$

33

Reservoir, geopressured Garg, $1978 \quad 14$ Isokrari, $1976 \quad 51$

Knapp, 1977

Tester, 1976

89

Reservoir, geothermal

Aamodt, 1973

Atkinson, 1977

80

Barelli, 1975

92

Celati, 1976

Chen, 1974

Cheng, 1974

Cheng, 1976

Faust, 1975

Garg, 1978

Hunsbedt, 1977

Kasameyer, 1975

Kruger, 1974

Lasseter, 1975

Lipman, 1977.

Marconcini, 1977

Martin, 1975

Mercer, 1974

Mercer, 1975

29

12

13

36

35

43

14

48

52

.55

56

95

Pritchett, 1975

Ramey, 1975

59,96

61

60

65

66

Reservoir, hydrothermal

Banwe 1 , 1964

Mercer, 1975

10

Nathenson, 1975

61

87

Reservoir, liquid-dominated Cheng, 1976 
SUBJECT/AUTHOR/YEAR

PAGE

Reservoir, liquid-dominated

(cont.)

Faust, 1976

Kasameyer, 1975

Nathenson, 1975

Pritchett, 1975

Reservoir monitoring

Banwe11, 1964

10

Reservoir, oil

Atkinson, 1977

Faust, 1975

R-Rivera, 1978

Reservoir parameters

Athertan, 1975

28

Reservoir simulation

Murphy, 1975

Tansev, 1978

Reservoir steam

Martin, 1975

59,96

Reservoir stimulation

Gould, 1975

Reservoir, vapor-dominated

Celati, 1977

Mercer, 1975

Moench, 1976

Nathenson, 1975

Pritchett, 1975

Reservoir, water-dominated

Chen, 1978

Mercer, 1975

Robinson, 1974

86

73

43

33

61

62

63

65

13

67

67

Reservoirs

Ferrara, 1970

Gringarten, 1976

94

45

Hunsbedt, 1976

Kubota, 1976

Miller, 1978

Schrock, 1976

Takahashi, 1975

Resistivity, electrical

Austin, 1977

Donaldson, 1977
SUBJECT/AUTHOR/YEAR

PAGE

Resistivity, electrical (cont.)

Sanya 1, 1974

Tezcan, 1975

Wooding, 1975

21

74

75

Rock

Bodvarsson, $1976 \quad 12$

Bodvarsson, $1977 \quad 31$

Nathenson, $1975 \quad 63$

Somerton, $1976 \quad 70$

Takahashi, $1975 \therefore 73$

Rock composition

Knapp, 1977

54

Rock compressibility

Bernard, 1975

Isokrari, 1976

11

51

Rock, fractured

Hsu, 1975

15

Rock fragments

Kuo, 1975

55

Rock, igneous

Kuo, 1975

55

Rock mechanics

Bodvarsson, $1977 \quad 31$

Browne 11, $1975 \quad 32$

Isita, $1970 \quad \because \quad 50$

Rock porosity

Kruger, 1975

Lapwood, 1948

55

56

Rock properties

Banwe 11, $1964 \quad 10$

Dudarev, $1970 \quad 40$

Faust, $1975 \quad 42$

Murphy, $1977 \quad 86$

Ramey, $1974 \quad 20$

Rock temperature

Sharma, 1975

89

Rock, volcanic

Aamodt, $1973 \quad 80$

Hunt, $1970 \quad 94$ 
SUBJECT/AUTHOR/YEAR

$\underline{\mathbf{S}}$

Sand, consolidated

Leverett, 1942

Sand, unconsolidated Cady, 1972

Morrison, 1949

Sandstone

Celati, 1975

Piwinski, 1977

Sinnokrot, 1971

Saturation

Bredehoeft, 1965

Kassoy, 1975

Leverett, 1942

Martin, 1975

Sinnokrot, 1971

Sondergeld, 1977

Tewhey, 1978

Toronyi, 1977

Scale deposits

Kubota, 1976

Scaling

Isita, 1970

R-Rivera, 1977

Screening

Tsang, 1978

Seismicity

Calamai, 1973

Sensitivity theory

Atherton, 1975

Shale

Hinrichs, 1975

Isokrari; 1976

Morse, 1978

Schroeder, 1976

Silica

Meidav, 1975

Simulation

Coats, 1974

Diadkin, 1975

Faust, 1975
28

PAGE

57

33

63

12

19

70

32

53

57

59,96

70

70

22

74

84

50

96

90

81

15

51

18

21

18

37

82

43
SUBJECT/AUTHOR/YEAR

PAGE

Simulation (cont.)

Faust, 1976

Morse, 1978

42

Pinder, $1975 \quad 64$

Rice, $1976 \quad 20$

Sato, $1975 \quad 88$

Takahashi, $1975 \quad 73$

Toronyi, $1977 \quad 74$

Weres, $1978 \quad 75$

Stability, convection

Sondergeld, $1977 \quad 70$

Wooding, 1960

76

Stability theory

Wooding, 1960

76

Steady-state

Balagna, $1976 \quad 10$

Cheng, $1974 \quad 36$

Cheng, $1976 \quad 35$

Tsang, $1975 \quad 90$

Wooding, $1957 \quad 77$

Wooding, $1957 \quad 76$

Steam

Baba, 1970

Budd, 1973

Cady, 1972

Celati, 1973

Coats, 1974

Donaldson, 1962

9

81

33

93

37

40

Ellis, 1964

93

Facca, 1964

Grant, no date $\quad 44$

Holst, $1972 \quad 46$

. Horne, $1978 \quad 15$

Hunsbedt, $1976 \quad 50$

Hunsbedt, $1978 \quad 48$

Moench, $1976 \quad 62$

Moench, $1978 \quad 18$

McFarland, $1976 \quad 85$

McNabb, $1975 \quad 59$

Rumi, $1970 \quad 21$

Yuhara, $1970 \quad 24$

Steam, dry

James, 1968

Tezcan, 1975

51

74

Stimulation

Aamodt, $1973 \quad 80$

Kruger, $1975 \quad 55$ 


\section{SUBJECT/AUTHOR/YEAR}

PAGE

Stimulation (cont.)

McKee, 1975

Murphy, 1975

Sharma, 1975

Strain

Rinehart, 1975

67

Stress intensity factors

Sarda, 1975

88

Stress waves

McKee, 1975

85

86

89

85

Stresses

Brownel1, 1975

32

Rinehart, 1975

67

Somerton, 1976

Subsidence

Bodvarsson, 1975

Hunt, 1970

Mercer, 1974

Papadopulos, 1975

Pinder, 1975

Tsang, 1978

Surface fatigue

Hsu, 1975

15

Surface pressure

Bazant, 1975

Surface surveys

Bernard, 1975

Surface tension

Cady, 1972

I

Temperature

Banwe11, 1964

Bodvarsson, 1974

Bolton, 1970

Calamai, 1973

Campbe11, 1978

Cataldi, 1970

Dudarev, 1970

Einarsson, 1975

Elder, 1970

Glover, 1970

40

82

40

94
SUBJECT/AUTHOR/YEAR

PAGE

Temperature (cont.) James, $1970 \quad 16$

Juprasert, $1977 \quad 84$

Mann, $1975 \quad 17$

Miller, $1978 \quad 62$

Nathenson, $1976 \quad 96$

Piwinski, $1977 \quad 19$

Scherer, $1975 \quad 69$

Tsang, $1975 \quad 90$

Tsang, $1978 \quad 90$

Yuen, 1977

24

Temperature gradient

Donalds on, $1962 \quad 40$

Facca, $1964 \quad 14$

Horton, $1945 \quad 48$

Tester, $1976 \quad 89$

Testing flow

Chen, 1978

13

Kunze, 1975

16

Testing well

Atkinson, $1977 \ldots 9$

Baba, $1970 \quad 9$

Banwe11, $1964 \quad 10$

Barelli, $1975 \quad 11$

Bernard, $1975 \quad 11$

Celati, $1975 \quad 12$

Chen, 1974

Chen, $1978 \quad 13$

29 Gould, $1975 \quad 43$

Gulati, $1975 \quad 15$

Kunze, $1976 \quad 16$

Laughi in, $1977 \quad 85$

Lipman, $1977 \quad 95$

Mann, $1975 \quad 17$

Marconcini, $1977 \quad 59$

Morse, $1978 \quad 18$

Narasimhan, $1975 \quad 19$

Rice, $1976 \quad 20$

Stoker, $1975 \quad 22$

Takahashi, $1975 \quad 73$

Tansev, $1978 \quad 73$

Yuen, $1977 \quad 24$

Texas

Gould, 1975

Knapp, $1977 \quad 54$
Thermal circulation Kononenko, 1974 


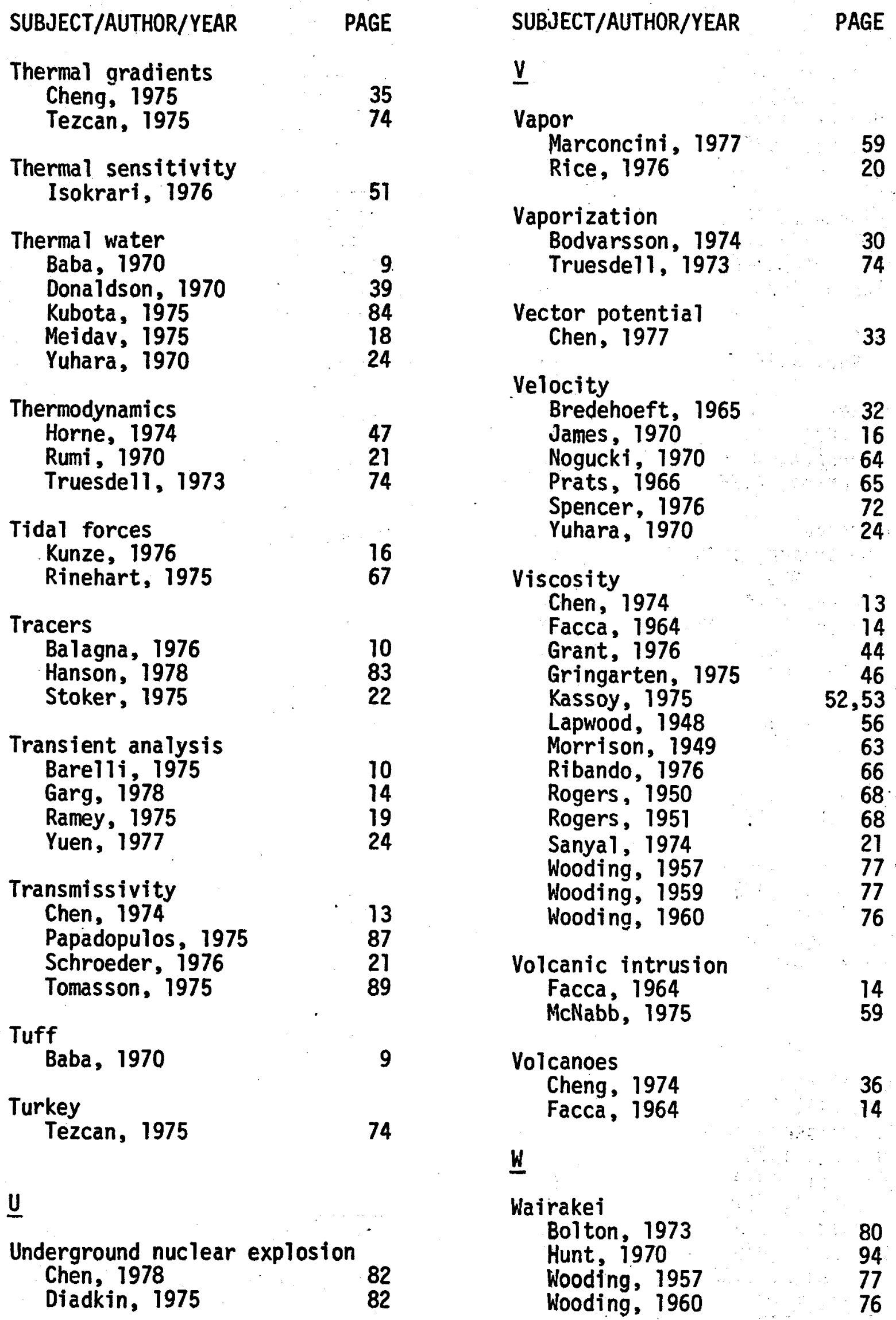


SUBJECT/AUTHOR/YEAR

PAGE

Water

$$
\begin{array}{ll}
\text { Grant, } & 1976 \\
\text { Kassoy, } & 1975 \\
\text { McNabb, } & 1975
\end{array}
$$

Water depletion

Celati, 1973

Water levels

Bolton, 1970

Brigham, 1977

Celati, 1978

Ellis, 1964

Water-steam effects

Browne 11, 1975

Water table

Barel1i, 1975

Celati, 1973

Cheng, 1973

Water temperature

Averiev, 1964

Mahon, 1970

Sharma, 1975

Water, waste

Caíama i, 1973

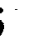

Einarsson, 1970

Waves, acoustic

Van der Knapp, 1959

23

We11 completion

Leverett, 1942

57

We11 design

Gould, 1975

Toronyi, 1977

Well diameter

Rumi, 1970

Campbe11, 1978

81
82

23

\section{7}

43

74

Well dimension

Well hole

Budd, 1973

Ridgway, 1977

87

88

Well. $\log$ analysis

Mann, 1975

17
SUBJECT/AUTHOR/YEAR

PAGE

Well output

$$
\begin{aligned}
& \text { Bolton, } 1973 \\
& \text { Papadopulos, } \\
& \text { We11 performance } \\
& \text { Coats, } 1977
\end{aligned}
$$

Papadopulos, 1975

80

87

36

Well spacing

James, 1970

Kubota, 1975

16

84

Well stimulation Bodvarsson, $1975 \quad 30$

Kunze, 1975

16

Wells, exchange

Scherer, 1975

69

Wells, geothermal

Averiev, 1964

Barel1i, 1970

Black, 1975

Campbel1, 1978

Garg, 1978

Gould, 1974

Juprasert, 1977

McFarland, 1976

R-Rivera, 1977

Ramey, 1962

Sharma, 1975

10

11

81

14

82

84

85

96

19

89

Wells, hydrotherma $]$

Garg, 1974

43

Wells, injection

Gould, 1975

Gringarten, 1975

43

Morse, 1978

Tsang, 1975

Tsang, 1978

46

18

90

90

Wells, oil

Ramey, 1962

20

Wells, production

Gringarten, $1976 \quad 45$

Kubota, $1975 \quad 84$

Morse, $1978 \cdots 18$

Nogucki, $1970 \quad 64$

R-Rivera, $1978 \quad 96$

Scherer, $1975 \quad 69$

$\begin{array}{ll}\text { Tsang, } 1975 & 90 \\ \text { Tsang, } 1978 & 90\end{array}$ 
AUTHOR/SUBJECT/YEAR

PAGE

Wells, reinjection

Kubota, 1976

Schrock, 1976

84

69
AUTHOR/SUBJECT/YEAR

PAGE

Wells, steam-dominated

Atkinson, 1977

9 